Portland State University

PDXScholar

Winter 3-23-2017

\title{
Microwave Instrumentation and Sensing Techniques for Quantum Efficiency and Minority-Carrier Lifetime Measurements
}

Kyle Benjamin Lu

Portland State University

Follow this and additional works at: https://pdxscholar.library.pdx.edu/open_access_etds

Part of the Electrical and Computer Engineering Commons Let us know how access to this document benefits you.

Recommended Citation

Lu, Kyle Benjamin, "Microwave Instrumentation and Sensing Techniques for Quantum Efficiency and Minority-Carrier Lifetime Measurements" (2017). Dissertations and Theses. Paper 3503.

https://doi.org/10.15760/etd.5387

This Thesis is brought to you for free and open access. It has been accepted for inclusion in Dissertations and Theses by an authorized administrator of PDXScholar. Please contact us if we can make this document more accessible: pdxscholar@pdx.edu. 
Microwave Instrumentation and Sensing Techniques for Quantum Efficiency and Minority-Carrier Lifetime Measurements

\title{
by
}

Kyle Benjamin Lu

A thesis submitted in partial fulfillment of the requirements for the degree of

\author{
Master of Science \\ in \\ Electrical and Computer Engineering
}

Thesis Committee:

Richard L. Campbell, Chair

Branimir Pejčinović

Erik Sanchez

Portland State University

2017 


\begin{abstract}
A non-contact method characterizing the quantum efficiency of a solar cell using the microwave reflectance signature is presented in this thesis. Traditional solar cell quantum efficiency $(\mathrm{QE})$ measurements are only possible near the completion of the fabrication process using contacts in direct physical connection with the metalized surface tabs to probe and extract charge carriers from the device. However, pressure within the solar metrology industry to report the spectral performance of the device earlier in the manufacturing process as part of the process control loop requires that a new non-contact method be developed. This thesis work contributes the development of a non-contact focused microwave reflectance technique capable of acquiring the full 365nm - 1100nm spectrum in under 1 minute. Unlike many similar Time Resolved Microwave Conductivity (TRMC) and Microwave Photoconductivity Decay ( $\mu P C D)$ systems based on the open-ended waveguide technique, this measurement is developed to perform measurements in the far-field. As such, a different mechanism for understanding the problem is presented using the modulated scatterer concept from antenna theory. Using a combination of high dielectric sensor pads and negative-index of refraction microwave lenses, we are able to tune the far-field field probe size from $5 \mathrm{~mm}-150 \mathrm{~mm}$ allowing for high speed single point in-line measurements or high spatial sensitivity laboratory measurements.
\end{abstract}




\section{Acknowledgments}

First I would like to thank my thesis advisor Dr. Richard Campbell helping to facilitate my internalization of the modulated scattering problem. Beginning with a prior research project on micropower scatterers meant to track oil slicks on the ocean surface, his guidance helped me to understand the problem from an entirely different perspective that I had not known to investigate. And while it took some time (perhaps, more than I would like to admit) for the idea to fully sink in, this thesis would not be what it is today without his guidance on this subject. I am also very thankful for our discussions of basic antenna theory and analog electronics.

I am also extremely grateful for Dr. Branimir Pejčinović's guidance during Phase 1 of the project as my faculty advisor for an industrial design course meant to teach students critical engineering skills from project planning, resource allocation, documentation, development/testing. This thesis evolved out of that coursework and would not have been possible without his guidance overseeing the first revision of hardware. More than this, however, I would like to sincerely thank Dr. Pejcinovic for his outstanding classes on introductory microwave theory and for the numerous hours that he spent with his students in lab.

To my collegues at Tau Science Corporation, I am indebted to you for your encouragement and unwaivering support of my educational goals. You have witnessed my struggle firsthand and I feel truly enriched to be working alongside such amazing people. Learning from your shared experiences is an extremely motivating and rewarding experience. I would like to sincerely thank 
Dr. Gregory Horner, Dr. Leonid Vasilyev and Mr. Antonious (Tom) Dirriwachter for their guidance over the course of this project.

To my friends and classmates, I thank you for your support and numerous discussions about the project. Chris Halseth, Rusty Wiseman and Nicholas Sayre all played critical design roles during Phase I of the project as part of our team-based industry design course. This work would not be where it is today without their combined efforts. And to my aspiring amature radio friends, I just got my ARRL membership with the call sign kb2l. One day soon, I will get an official FCC license and we will work our RF magic to talk to each other over the airwaves!

Finally, I would like to give a shout out to my parents Lorna and Randy for their love, support and 12 years of homeschooling! Love you guys, thanks!

This thesis work contributes the development of a non-contact quantum efficiency measurement technique using the far-field microwave reflectance signature and is supported by Tau Science Corporation with funding, in part, by the Department of Energy SBIR Grant \#DE-FG02-12ER90434. 


\section{Contents}

$\begin{array}{ll}\text { Abstract } & \text { i }\end{array}$

$\begin{array}{ll}\text { Acknowledgments } & \text { ii }\end{array}$

Contents $\quad$ iv

List of Tables $\quad$ vi

List of Figures $\quad$ viii

1 Introduction $\quad 1$

1.1 The Process Control Motivation . . . . . . . . . . . . . . . 1

1.2 Thesis Statement . . . . . . . . . . . . . . . . . . 4

1.3 Background Literature . . . . . . . . . . . . . . 5

1.3.1 Microwave Detected Lifetime Measurements . . . . . . . . 5

1.3.2 Microwave Detected Electric Field Measurements . . . . . 6

1.3.3 Negative Index of Refraction Metamaterials . . . . . . . 7

1.4 Thesis Overview . . . . . . . . . . . . . . . 8

2 The Study of Microwave Reflectance 12

2.1 Methods and Techniques Overview . . . . . . . . . . . . . . . . 12

2.2 Microwave Reflectance Argument \#1 . . . . . . . . . . . . . . . . . 14

2.2.1 Plane Wave Reflection . . . . . . . . . . . . . . . 15

2.2.2 Perturbation of the Microwave Reflectance . . . . . . . . . 17

2.2.3 The Effect of Imhomogeneous Conductivity Profiles on Lifetime Measurements . . . . . . . . . . . . . 19

2.2.4 The Effect of Imhomogeneous Conductivity Profiles on QE Measurements . . . . . . . . . . . . . 22

2.2.5 Non-Uniform Microwave Response Due to Phase Shift . . 23

2.3 Open-Ended Waveguide Measurement . . . . . . . . . . . 25

3 The Modulated Scattering Problem 27

3.1 Basic Scattering Technique . . . . . . . . . . . . . . . 28

3.1.1 Classes of Scatterers . . . . . . . . . . . . . . . . . 30

3.1.2 Unmodulated Scattering . . . . . . . . . . . . . 30

3.1.3 Electrically Modulated Scatterer . . . . . . . . . . . . . 33

3.1.4 Optically Modulated Scatterers . . . . . . . . . . . . . 37

3.2 Microwave Reflectance Argument \#2 . . . . . . . . . . . . . . . . 39

3.2.1 Ideal Dipole Antenna . . . . . . . . . . . . . . 40 
3.2.2 Radiansphere . . . . . . . . . . . . . . . . . . 42

3.2.3 Consideration of Induced Currents in a Dielectric Material 42

3.2.4 Modulation of a Microwave Field by Dipole Scatterer . . . 43

3.3 Solar Cell as an Aperture Antenna . . . . . . . . . . . . . . . . 44

4 Performance of a Solar Cell 48

4.1 Spectral Binning of Solar Cells . . . . . . . . . . . . . . . 48

4.2 Standard QE Measurement Technique . . . . . . . . . . . . . . 50

4.2.1 Contact QE Curve Analysis . . . . . . . . . . . . . . . 51

5 Systems Level View 54

5.1 Microwave Reflectometer . . . . . . . . . . . . . . 55

5.2 Hardware Implementation . . . . . . . . . . . . . . . . . . 58

5.2 .1 Light Engine . . . . . . . . . . . . . . . . . 58

5.2 .2 Optical Column . . . . . . . . . . . . . . . . . . . 61

5.2.3 Supporting Baseband Electronics . . . . . . . . . . . . . 62

5.2.4 Stage Control \& Map Generation . . . . . . . . . . . . . . . 64

5.2.5 Microwave Lens \& Antenna Fabrication . . . . . . . . . . . 65

5.2.6 Microwave Cavity Configuration . . . . . . . . . . . . 72

5.3 Software Control System . . . . . . . . . . . . . . . . 76

5.3.1 Measurement Flow Chart . . . . . . . . . . . . . . . 78

5.3 .2 Software Configuration . . . . . . . . . . . . . 80

5.3.3 System Calibration . . . . . . . . . . . . . . . . . . . 80

5.3.4 First Test Measurement . . . . . . . . . . . . . . . 82

6 Results \& Analysis 85

6.1 System Repeatability Measurements . . . . . . . . . . . . . . . 87

6.2 Quantum Efficiency . . . . . . . . . . . . . . . . . . . . . . . . 89

6.2.1 QE Response with Optical Filters . . . . . . . . . . . . . . 89

6.2.2 QE Response with Applied Bias . . . . . . . . . . . . . 99 90

6.2 .3 QE Maps . . . . . . . . . . . . . . . . . . . . . . . 992

6.3 Microwave Detected Minority-Carrier Decay Maps . . . . . . . 96

6.4 Amplitude vs Decay Maps . . . . . . . . . . . . . . . . . . . . 98

6.4.1 Sample 2 Characterization . . . . . . . . . . . . . . . 98

6.4.2 Sample 7 Characterization . . . . . . . . . . . . . . . 99

6.4.3 Low-Injection and High-Injection Considerations . . . . . 102

6.5 Photoluminescence Imaging vs Decay Maps . . . . . . . . . . . . 103

6.6 High Resolution Defect Scan . . . . . . . . . . . . . . . . 107

6.7 Application of High Dielectric Probe . . . . . . . . . . . . . . . . 108

6.8 Summary of Reference Images _. . . . . . . . . . . . . . . 110

6.9 Summary of Decay Maps . . . . . . . . . . . . . . . . . 111 
7 Conclusion $\quad 112$

7.1 Future Work . . . . . . . . . . . . . . . . . . . 114

$\begin{array}{ll}\text { References } & 117\end{array}$

Appendix A: Electroplating Process 122

7.2 Method 1: Custom Process . . . . . . . . . . . . . . . 122

7.3 Method 2: Airbrush Process . . . . . . . . . . . . . . 124

$\begin{array}{ll}\text { Appendix B: Poster Summary } & 125\end{array}$

Appendix C: Measurement Configuration Settings 126 


\section{List of Tables}

5.1 Summary of system components for non-contact microwave QE measurement instrumentation. . . . . . . . . . . . 55

5.2 Summary of measurement repeatability with stacked weak leds. 60

5.3 Summary of map resolution and timing generated from sample size and desired resolution. These values assume a standard 156mm x 156mm silicon sample and 17 unique wavelengths. . . 65

5.4 Summary of resistivity measurements results for copper coated antenna components using a 4 point probe. . . . . . . . . 71

6.1 Summary of experiments and result figure numbers for noncontact microwave reflectance measurement system. . . . . . . 86

6.2 Summary of measurement repeatability for the microwave detected quantum efficiency system as a function of wavelength. The SNR is calculated using $1 \sigma$ noise. . . . . . . . . . . . 88

6.3 Table of measured probes and the fundamental mode cutoff frequency for a circular waveguide. . . . . . . . . . . . . 109 


\section{List of Figures}

1.1 Solar Panel Model: An array of series and parallel solar cell elements are connected together to form a solar panel. Defective cells in the series component block current from contributing to the rest of the panel. . . . . . . . . . . . . . . .

2.1 Hardware similar to this is configuration is used for all microwave conductivity measurements. The sample is usually positioned over the mouth of an open-ended waveguide. However, some configurations place the sample inside of the waveguide behind an adjustable short circuit. A circulator differentiates between the incident and reflected power signals. . . . . . . . . . 26

3.1 Basic monostatic modulated scatterer configuration. . . . . . . . . 29

3.2 Basic monostatic scatterer problem described by Justice and Rumsey [1]. . . . . . . . . . . . . . . . . . . . . .

3.3 The modulated scatterer coherent detection method pioneered by Richmond [2]. . . . . . . . . . . . . . . . . . . . . . .

3.4 We assume that a dipole radiator is used to uniformly illuminate the sample with microwaves. The dipole is oriented on the $\mathrm{z}$ axis and the sample is assumed to lay in the $y$-z plane. . . . . . . .

3.5 The effective aperture of the uniformly illuminated solar cell is the area of the optical probe. Librated charge carriers are free to oscillate in phase with the incident E field. A new current is induced on the sample due to both the incident electric field and liberated charge carriers. . . . . . . . . . . . .

4.1 The quantum efficiency curve is a single variable function of the incident photon energy. Ideally, any photon with sufficient energy (i.e. $>1.12 \mathrm{eV}$ in silicon) to generate a charge-hole pair should be available to do work if an external circuit is connected. Sample defects preventing the carriers from being extracted will lower the overall quantum efficiency. . . . . . . . . . . . . . 50

5.1 System Diagram: This high level view of the measurement system illustrates all of the major components used to acquire the QE/lifetime data. . . . . . . . . . . . . . . 56 
5.2 Microwave Receiver: A block diagram of the $10.5 \mathrm{GHz}$ homodyning receiver used to both illuminate the solar cell with microwave energy and demodulate the reflected AM tone from the optically modulated solar cell. A digitally controlled source sets both amplitude and frequency $\ldots . \ldots \ldots \ldots \ldots$

5.3 Spectral Resolution: A typical LED spectrum showing available wavelengths before optical insertion losses in the fiber optic and scrambler. Power balancing is required to improve detection efficiency on weak signals as well as audio frequency dynamic range requirements. The light engine is designed to support repetitive LED channels to boost power output of the weakest LEDs. Convolution with a typical solar cell quantum efficiency curve indicates that optical power should be balanced around $20 \mathrm{~mW}$ to maintain constant signal-to-noise ratio across the spectrum for the current setup. . . . . . . . . . . . . .

5.4 Light Engine: 64 independent solid state light sources are packed into a fiber optic bundle and mixed inside of an optical scrambler to homogenize the individual channels. The scrambler is coupled to a collimating lens via an adjustable spot size aperture. Approximately $4 \%$ of the collimated signal is picked-off as a reference signal to monitor the incident number of photons hitting the sample. Finally, an output lens focuses the light back into a spot at the sample plane. . . . . . . . . . . . . . .

5.5 DAQ Electronics: The first revision of electronics was designed to accommodate four channels with a sampling rate of 1MSPS per channel. Data is streamed out over high speed USB proto$\mathrm{col}$ at $480 \mathrm{Mbit} / \mathrm{s}$. Measurement of a few solar cell samples experimentally demonstrated that the sampling rate requirement could be relaxed slightly. A second revision of hardware using the same processor in a leaded package and with a more versatile ADC was also fabricated. . . . . . . . . . . . . . . .

5.6 Microwave Lens: The final microwave lens was fabricated using a Form 1+3D printer and homebrew electroplating tank [?]. . . .

5.7 Lens Focal Lenght: The focal length of the lens is frequency dependent. Here we collected data near the standard operating point of $10.5 \mathrm{GHz}$. Depth position is given in centimeters away from the center of the lens. . . . . . . . . . . . . .

5.8 Electroplating Process: This flowchart describes two electroplating processes used in the construction of the microwave lens and horn antenna. Both methods are found to be completely successful although Method \#2 has a distinct speed advantage when the surface area of the object being coated is large and shallow. . . . 
5.9 Custom Horn Characterization: The optimal recorded coating and performance measurement was observed when using only copper conductive spray without electroplating process. $S_{11}$ is less than $-18 \mathrm{~dB}$ across the entire $\mathrm{X}$-band range. Circled in red is a consistently recurring defect visible in all 3D printed objects. In this case, the defect is out of band and can be safely ignored. . 71

5.10 Custom Horn Antenna Pattern: Measured antenna pattern with amplitude in $\mathrm{dB}$ and $\mathrm{x}$-variable in degrees. . . . . . . . . . .

5.11 Cross section of a waveguide probe examined by Richmond and Tice to improve near-field measurement accuracy [3]. Increasing the dielectric constant increases the cutoff wavelength of the fundamental mode by the square root of the relative permittivity. In the waveguide configuration shown here, mycalex $\left(\epsilon_{r}=11.5\right)$ is used as the dielectric filler. . . . . . . . . . . . . . . . .

5.12 Shaped waveguide dielectric antennas were not considered at the onset of this project. Here we settle for a cylindrical waveguide cavity. . . . . . . . . . . . . . . . . . 75

5.13 Left: A cylindrical waveguide cavity completely filled by a polystyrene dielectric $\left(\epsilon_{r}=4.2\right)$. Middle: A partially filled cylindrical waveguide cavity with the normal vector facing the microwave antenna. Alumina $\left(\epsilon_{r}=10.4\right)$ is used as the dielectric. Right: The top surface of the partially filled waveguide cavity facing the bottom of the solar cell. $5 \mathrm{~mm}, 7 \mathrm{~mm}$ and $13 \mathrm{~mm}$ dielectric cores are confirmed to provide sufficient coupling for either a $\mathrm{QE}$ or lifetime measurement. . . . . . . . . . . . . . . .

5.14 Measurment Subprocesses: QE and lifetime measurement processes are described in detail. After the map is complete the results are stored as pickled python objects for later review. In the case of the lifetime measurement, only the frame average, pulse amplitude and decay time subsets are saved. Map recipes are generated prior to the start of a measurement using the custom MapGenerator.py tool. In the event of an unexpected termination, all data currently stored in memory is copied to disk. . . . .

5.15 Software Components: The major measurement configuration software blocks are shown. All software components can be run and tested independently. Each module is accessed through a well defined class interface. . . . . . . . . . . . . . . . . . 
5.16 First Pass QE Measurement: This figure shows the initial test involving the full integration of both software \& hardware components. A UV bank of LEDs is loaded into the light engine and with an appropriate software configuration file indicating the wavelength, nominal drive current, and pulse repetition frequency. These measurements were taken in rapid succession to demonstrate measurement stability after calibration to a flat line at $60 \%$ EQE. . . . . . . . . . . . . . . . . . .

5.17 Thermal Drift: (Left) The system calibration curve. (Right) A second pass measurement with all four banks of wavelengths loaded into the light engine. Here, we perform continuous measurements of the same sample and position over a 30 minute period. This frame represents a measurement around 25 minutes into the data set and we can notice that thermal drift impacts system stability quite drastically. This issue is now resolved as confirmed by the system repeatability measurements in the following chapter. . . . . . . . . . . . . . .

6.1 Optical Filter Measurement: This measurement verifies that channel crosstalk, ground loop coupling and radiative coupling to the light engine are not observed in the data. The microwave detected signal is only observed as a function of the photogenerated carriers in the sample. The nominal QE response without any filter is shown in blue. The transfer function of each successive filter is plotted in green and the measured response shown in red. This data is acquired using the NIR lens with $1.5 \mathrm{~cm}$ diameter optical probe. . . . . . . . . . . . . . .

6.2 Optical Bias Measurement: The effect of a DC forward optical bias in QE measurements is significant. Starting from the lower left QE curve in clockwise fashion, an optical bias of 13\%, 20\%, $39 \%, 43 \%, 48 \%, 65 \%, 74 \%$, and $81 \%$ of 1 Sun illumination is applied to the sample. Two effects are observed here; 1) photoflattening in strong forward bias and, 2) low dark measurement sensitivity. . . . . . . . . . . . . . . .

6.3 1D Optical Bias Measurement: A slice of the biased quantum efficiency curves along the 405nm axis profiles the QE curve as a function of DC optical bias. The optical bias shifts the local operating point of the cell along the JV characteristic. . . . . . . . 
6.4 Quantum Efficiency Map: Three wavelength (UV-VIS-IR) slices of a QE dataset acquired on Sample 5 show strong spatial between the ultraviolet and visible wavelengths. Five measurement artifacts are observed and marked by black circles. The map resolution is $3 \mathrm{~mm}$ per pixel. . . . . . . . . . . . . . . .

6.5 Quantum Efficiency Map: Inverting the color scaling of Figure 6.4 (Sample 5) highlights additional cell defects as indicated by the ROI boxes. Defects shown persist in all layers of the measurement indicating a severe problem. Within the ROI of the lower right hand defect, we observe a $20-30 \%$ loss in available carriers across all wavelengths between the defect center and the edge of the ROI. . . . . . . . . . . . . . . . . . . .

6.6 Quantum Efficiency Map: Three wavelength (UV-VIS-IR) slices of a QE dataset acquired on Sample 10 with a $3 \mathrm{~mm}$ map resolution. The highlighted boxes indicate wafer handling issues while underperforming regions indicate potential issues with the deposition process. Thick boxes along the lower $1 / 3$ rd portion of the sample show skid marks along the direction of travel when the cell is moved from station to station. The lighter boxes indicate scuff marks and scratches working inwards from the edge of the sample. This sample is revisited in PL, decay and high resolution amplitude maps later in this chapter. . . . . . . . . . 96

6.7 Wideband detection of lifetime decay curve. Since the signal is known to be periodic, we break apart the $\mathrm{N}$ pulse frames in (a) and stack them up into a single pulsed average shown in (b). The Levenberg-Marquardt exponential fit algorithm is then applied to extract the decay time. . . . . . . . . . . . . . .

6.8 Amplitude vs. Decay Map: Comparison of the microwave reflectance signature for the decay (left) and amplitude (right) of Sample 2. Both images are derived from the same dataset showing distinct separation of amplitude and decay term defects. . . . 100

6.9 Decay and Amplitude Comparison: The microwave reflectance signature for the decay (left) and amplitude (right) of Sample 7 is shown. In this instance, the decay algorithm appears to be more robust than the amplitude map at identifying defects. We note that the decay map is able to extract useful information from inside the dead region of the amplitude map. . . . . . . . . . . 101

6.10 The JV curve is useful for showing the operating region for the two different measurements. The photoluminescence measurement occurs under strong forward bias conditions with an illumination intensity often greater than 1 Sun. . . . . . . . . . . 102 
6.11 Slight optical bias is used to highlight the trapping effect. Slight increase in bias illumination begins to fill trap locations resulting a more uniform device behavior. The sample on the left is shown with an increased broad spectrum white light bias source. . . . . 103

6.12 Decay \& PL Comparison: We compare a PL and time-resolved decay map for Sample 1 in this figure to identify cell specific defects. Sample 1 is quite noisy across the entire map. The measured decay times are pushing the limits of the system resolution. One defect along the right edge of the sample does clearly track the defect uncovered in the PL image. . . . . . . . . . . . . . 104

6.13 Comparison of PL and time-resolved decay map for Sample 2. . 105

6.14 Comparison of PL and time-resolved decay map for Sample 5. . 106

6.15 Comparison of photoluminescence and microwave reflectance amplitude data. The photoluminescence data was taken at 1MP resolution with a relatively weak excitation source. The map is recorded using a $4 \mathrm{~mm}$ step size along both the $\mathrm{x}$ and $\mathrm{y}$ axes. . . 107

6.16 A high resolution scan of the defective corner shown in Figure 6.15 shows many distinct scratches running over the surface of the sample coinciding with the affected areas in the corresponding photoluminescence image. . . . . . . . . . . . . . . 108

6.17 This figure shows three different probe styles applied directly underneath the sample. The three probe sizes are $5 \mathrm{~mm}, 7 \mathrm{~mm}$, and $13 \mathrm{~mm}$. . . . . . . . . . . . . . . . . . . . . . . . . . . 109

6.18 Summary of reference photoluminescence images for the sample set. Samples were biased to approximately 1 Sun with a pair of high power 528nm (green) LEDs. Top Row: Samples \#1,\#2,\#3; Bottom Row: Samples \#4,\#5,\#10 . . . . . . . . . . . . . . . . 110

6.19 Summary of time-resolved decay maps. All decay times were calculated using the Levenberg-Marquardt exponential fit model. The signal-to-noise ratio of Sample 1 proved too small to reliably extract meaningful minority-carrier decay information. Samples 2-5 each recorded consistent decay constants on the order of $75 \mu \mathrm{s}$. Measurements on sample 10 recorded a slightly lower decay time constant of approximately $55 \mu \mathrm{s}$. Top Row: Samples \#1,\#2,\#3; Bottom Row: Samples \#4, \#5,\#10 . . . . . . . . . . . . 111

7.1 A localized short-circuit current experiment using bias lasers to suppress the junction of the device . . . . . . . . . . . 116

7.2 Team project for an industrial design course at Portland State University, 2015 . . . . . . . . . . . . . . . . . . . . . . . . 125 


\section{Introduction}

Quantum efficiency measurements tracking the ratio of photogenerated charge carriers to the number of incident photons as a function of photon energy is one of the fundamental tools available to engineers assessing solar cell device performance. Detailed analysis of quantum efficiency (QE) curves yield a nontrivial amount of quantitative information regarding the short-circuit current $J_{s c}$ output of the cell, front and rear surface recombination factors as well as many subsurface defects. If equipped with a reflectance detector and $x-y$ scanning stage, QE measurements mapped across the surface of the sample yield additional information about surface reflectivity, sheet resistance, cracks and wafer handling defects introduced during the production process. Optical DC bias sources may also be employed to study the severity of front or rear surface traps found by a standard QE measurement while electrical bias is often used to study the QE curve at different operating points along its current-voltage characteristic. The quantum efficiency measurement is therefore quite substantive in its report of under-performing cells and can successfully identify a broad range of surface and subsurface defects limiting the overall power generation capability of the device.

\subsection{The Process Control Motivation}

Given the level of detail available from a quantum efficiency based analysis, a few questions immediately come to mind. (1) Why is the QE measurement not 
a standard technique used on the production line? (2) What gains should be expected by folding the spectral information into the process control loop? (3) Why is this a valuable topic of research for the solar community in general?

Addressing the first question, quantum efficiency measurements usually occur near the end of the production process after current carrying busbars and fingers have been deposited on the surface of the sample. Exposed surface metalization and electrical tabs allow charge carriers liberated on one side of the sample to be swept out into an external circuit connected to the other side of the sample with minimal losses. Without these contacts, it would be impractical to perform a quantum efficiency measurement since physical probes would need to make contact with a number of high resistance points across the cell introducing another potential failure mode. Furthermore, traditional QE measurements are impractical in-line as the full spectrum from $300 \mathrm{~nm}$ to $1100 \mathrm{~nm}$ can take upwards of 20 minutes to complete each measurement site. If the production line processes 2400 cells $/ \mathrm{hr}$, the QE cell sorted will only provide a less than $1 \%$ coverage of the total cell yield. A useful QE in-line measurement must resolve both the probe contact and measurement speed issue before it will ever have an appreciable impact on reliability performance and production yield.

Considering that the average silicon solar cell quantum efficiency is already well above 90\% mark in the peak power generating portion of the AM1.5 spectrum, we might ask why a spectral binning measurement is even needed. This concern is best addressed with the help of the simple solar cell and solar panel models shown in Figure 1.1. The solar cell is modeled as an ideal current source connected in parallel with a diode and shunt resistance. Series resistance between the device and external world also receives some attention in 


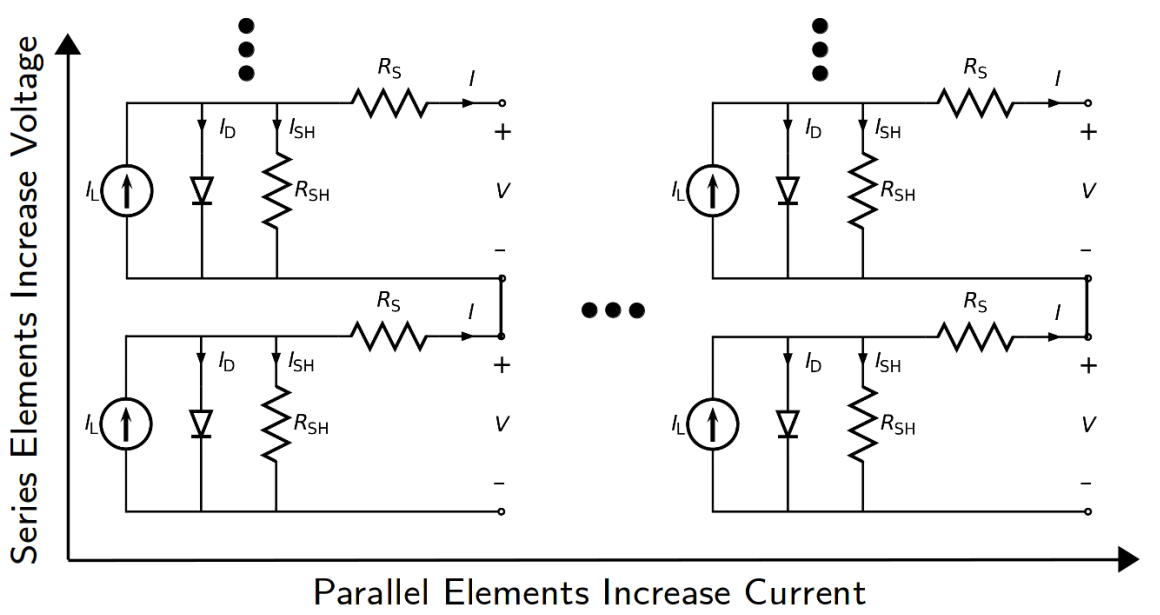

Figure 1.1: Solar Panel Model: An array of series and parallel solar cell elements are connected together to form a solar panel. Defective cells in the series component block current from contributing to the rest of the panel.

the standard model. Photogenerated current produced by the solar cell as light illuminates the wide area surface is swept out of the device where it can do work on an external circuit. Strings of solar cells are connected together to boost the total voltage output across the terminals of a solar module while parallel solar cell strings contribute to the total current output of the module.

With this series-parallel view of the solar module, it should be apparent that any reduction of device performance of a single series-element cell must also detrimental to the entire string of cells. Module performance is severely impacted by mismatches in current output with high performance cells contributing no more than the worst performance device in the chain. And while cell sorters can current bin devices using flash testers for JV analysis, all information about the spectral component of the light is ignored. Since the intensity of the light spectrum reaching the surface of the Earth changes with the time 
of day and the solar elevation, current matching between devices is not guarenteed throughout the day. For this reason, a calibrated spectral measurement of every device is desirable since it allows optimal current matching between devices from morning to dusk maximizing the total power generation of the device per day.

Let's bring it all together; why is the solar community interested in maximizing power in the wee hours of the morning and late evening? The first answer is that spectral binning gives solar manufactures an edge over the competition when they are able to generate more dolars per square meter of surface area. The second and perhaps more important aspect is that current matching reduces failure modes and improves module reliability in the field. As the marketable module warranty can exceed 25 years, it is critical that all devices operate consistently throughout their entire lifespan. Therefore, there is increasing pressure across the solar industry to develop a non-contact spectral binning technique that is capable of binning cells prior to the metalization phase of processing in order to save both time and materials from being wasted on a poorly performing device.

\subsection{Thesis Statement}

A non-contact metrology technique capable of characterizing the quantum efficiency and minority-carrier lifetime of a solar cell prior to the surface metalization phase of processing is possible using the far-field microwave reflectance signature of the device when it is optically modulated by a $20 \mathrm{~mW}$ broad-spectrum solid state light engine. 


\subsection{Background Literature}

The microwave reflectance technique applied in this thesis to the study of quantum efficiency and minority-carrier lifetime is rooted in the joint application two distinct fields of study: (1) the study of minority-carrier lifetime in semiconductors and, (2) the study of electric field distributions in the vicinity of a strong electromagnetic radiator. Our goal for the remainder of this section is to narrow our focus of these two important bodies of work to provide context for the non-contact quantum efficiency measurement that will be discussed throughout this work. However, a slight deviation from the background narrative is necessary to include the work leading into the fabrication of a negative index metamaterial lens used to acquire data in this work.

\subsubsection{Microwave Detected Lifetime Measurements}

In 1952, Hall, Shockley and Read laid the theoretical foundation describing the statistical process of electron-hole recombination affecting virtually all semiconductor devices $[4,5]$. Time-resolved measurements of the excess electronhole recombination due to various recombination processes are particularly adept at detecting material defects in semiconductors and have resulted in the publication of over 300 papers, covering 35 different methods, in the following 15 years of work [6]. Non-contact measurements capable of accurately describing the behavior of carrier lifetime in semiconductors using the transmission of microwaves through a sample were first reported in 1959 [7]. Shortly afterwards, several articles reported the ability of a microwave reflectance techniques capable of accomplishing the same goals in 1962-1963 [8-10]. 
These microwave detected lifetime studies were revived and advanced significantly by Kunst and Beck in 1986-1987 when they succinctly reported on the general theory of excess conductivity induced by pulsed laser excitation in the microwave frequency range [11]. The relationship between excess carrier concentration and the microwave reflectance signal is derived in the same paper. In a paper published in the following year, they extend their theory to take into account the dependence of microwave reflectance on the choice of probe frequency and the dark conductivity of the sample [12]. Both papers demonstrate the use of a robust circulator based open-ended waveguide apparatus to collect extensive datasets proving the validity of their claims. Later studies by Schofthaler and Brendel, and more recently Ahrenkiel and Johnston demonstrate multilayer modeling, relationship between reflectance and sample thickness, mirror reflectance, and high-injection lifetime measurements in the transmission mode of microwave spectroscopy $[13,14]$.

\subsubsection{Microwave Detected Electric Field Measurements}

In the same time period as the initial foray into semiconductor characterization there was a second large body of work being performed on the characterization of electric field distributions due to electromagnetic horn antennas. Measurements of these distributions in free space were of particular importance to antenna engineers involved in the design of early warning radar systems during World War II. The normal method for characterizing electric field distributions was to use a pair of reciprocal antennas to probe the point of interest. However, this method was challenging to setup and the results were often not repeatable. In 1954, Justice and Rumsey propsed an alternate detection method using the 
microwave reflection off of a scattering probe [1]. The main attraction of this technique is the elimination of an interferring transmission line between the probe and receiving electronics. In the following year, Richmond proposed a modification to the scattering technique by introducing a modulated signal at the obersvation point of the probe [2]. The new technique proved capable of significantly reducing the RF isolation requirements while providing some immunization against externally induced distortions. Later that year, Richmond and Tice published a follow-up paper on optimal probe design demonstrating the use of tapered waveguides filled with high dielectric materials to minimize the detection area and discriminate between linearly cross polarized waves [3]. The major improvements proposed by Richmond in these two papers serve as the foundation for all modulated scattering techniques to follow. In 1973, Dyson wrote a definitive review highlighting the techniques used for the measurement of near field antennas and scatterers up to that point [15].

\subsubsection{Negative Index of Refraction Metamaterials}

The mathematical theory and strange implications of doubly-negative metamaterials were first reported on in depth by Veselago in 1968 [16]. He reported that such materials, although not found in nature, were not explicitly prohibited by the physical laws of the universe. He also reported that a material with these strange characteristics would be capable of creating lenses that could exceed the classical far-field diffraction limit. The extent of his work was largely un-noticed for many years until the publication of two popularizing APS Physical Review Letters by Smith et al demonstrating a practical NIR metamaterial using split-ring resonators and Pendry who showed mathematically that NIR 
metamterials have the addition property of providing amplification of the nearfield evanescent waves at the image plane thus allowing the creation of the 'perfect lens' in the year $2000[17,18]$. After these two developments, much of the recent work on negative index metamaterials has focused on the practical design, fabrication and characterization of metamaterial structures in the $\mathrm{GHz}, \mathrm{THz}$, and optical frequency bands [19]. Today, negative index metamaterials have reached sufficient maturity in the literature to merit integration into wider fields of study, but are, to this point, still widely underutilized by the academic and industrial community. In this thesis, we report on the fabrication of a low cost NIR lens using designs published in a recent 2012 journal article [20]. We also report on the fabrication of a low cost $\mathrm{x}$-band horn antenna using a different metalization technique. Both parts are considered part of the instrumentation and necessary for the data collection phase of this thesis.

\subsection{Thesis Overview}

In the following chapters, we develop non-contact instrumentation at 8-12 GHz that is capable of scanning across the surface of silicon solar cells to extract the quantum efficiency and minority-carrier lifetime prior to the surface metalization phase of processing. The modulated scatterer concept from antenna theory sets the framework for which we view and think about the problem. These trajectory will go as follows:

- Chapter 2 begins with a discussion of two different perspectives of the microwave reflection problem. The first perspective studies at microwave reflections due to the modulated photoconductivity from a sample positioned across an open-ended waveguide in the near-field. The second 
perspective describes microwave reflections in both the near and far-fields using an application of antenna theory known as the modulated scatterer. The two techniques are fundamentally related by the definition of conduction current and a direct application of the antenna reciprocity theorem allows us to apply antenna theory concepts to the study of photogenerated carriers in a semiconductor. After presenting both techniques, the remainder of the chapter is devoted towards covering the first technique in greater detail.

- Chapter 3 introduces the modulated scatterer technique (MST) using both electrically and optically modulated scatterers. A review of the major advances to the MST from the primary literature is used to inform our design specifications for the homodyning receiver required to detect the changes in sample photoconductivity. Electric field probe geometries from the literature are reviewed and new probes for this thesis are devised. Huygens' principle and the reciprocity theorem from antenna theory are discussed and we propose an alternative approach for thinking about the reflectance problem. Emphasis in this chapter is placed on instrumentation and signal detection methods.

- Chapter 4 very briefly introduces the standard contact style quantum efficiency measurement technique. From there, we identify several regions of interest in the blue, bulk and red quantum efficiency response. Quantitative information from the non-contact measurement is possible with a calibrated system. For this thesis, we focus on the qualitative information that can be garnered from the non-contact data since a calibration sample is not presently available. 
- Chapter 5 covers the entirety of the instrumentation design and software development aspects implemented in this thesis. The hardware section covers the design of a custom high-speed USB data acquisition system, microwave receiver, light engine, optics, motion control, and RF enclosure. A detailed disucssion is provided on the fabrication and testing of a microwave NIR lens and $\mathrm{x}$-band horn antenna based on 3D printing technology and electroplating technology. Separate flow diagrams of the measurement process and major subprocesses charts in the software development are discussed.

- Chapter 6 covers the measurement setup, calibration, acquisition, data analysis and system performance review. Custom solar cell samples pulled from a manufacturing line prior to the metalization phase of processing are studied. Quantum efficiency measurements, minority-carrier decay maps, and photoluminescence (PL) images are studied. Maps of the minority-carrier decay demonstrate unique features not visible in amplitude maps of the same sample.

- Chapter 7 offers concluding remarks and illustrates several areas of the study that might be covered in future work. These areas primarily focus on the relationship between antenna theory and the impedance mismatch model. However, phase response data, 3D tomographic and additional experiments for improved signal sensitivity are proposed.

- Appendix A: The appendix covers the electroplating steps that were taken during the fabrication of the microwave lens and horn. 
- Appendix B: A short poster summary detailing the Author's prior work with Nicholas Sayre, Chris Halseth and Rusty Wiseman as part of Portland State University's 2015, ECE Industrial Design Course.

- Appendix C: Example application configuration file to configure the displayed plots, light engine, data acquisition system, calibration curves and motion control system. 


\section{The Study of Microwave Reflectance}

\subsection{Methods and Techniques Overview}

Depending on the reader's inclination towards either the physics or engineering journals, the problem can be approached along one of two possible avenues of research. In an attempt to assemble a congruent picture of these two ideas side-by-side and bring the relevant terminology to bear, let's consider how the problem is viewed from each vantage point. A review of the available literature beginning with Justice and Rumsey [1] on scattering field measurements and Ramsa et. al. [7] on microwave detected lifetime measurements shows a distinct favorability towards the IEEE: Antennas and Propagation family of journals and the Journal of Applied Physics respectively. The two methods are described as follows:

- Method 1-Journal of Applied Physics: The microwave reflectance signature of a semiconductor target is studied as a function of the change in surface conductivity due to excess charge carriers liberated by a pulsed laser. Time-resolved measurements of the minoirty-carrier lifetime $(\tau)$, or the $1 / e$ decay time of excess minority-charge carriers, is a fundamental material parameter of semiconductors used in virtually all related semiconductor fields. Time-resolved measurements are also used to study wafer conductivity, carrier mobility, dopant concentration and surface recombination velocities. The crux of the conductivity argument is that an impedance mismatch between the sample and microwave system must 
cause a signal to be reflected back towards the receiver. Thus a perturbation of sample conductivity, and therefore sample impedance, via the liberation of excess charge carriers must produce a small signal change in the microwave reflectance signature.

keywords: Time-resolved microwave conductivity (TRMC), microwave photoconductive decay ( $\mu \mathrm{PCD})$, microwave detected minority-carrier lifetime $(\tau)$, laser induced microwave absorption

- Method 2-IEEE: Antennas and Propagation ${ }^{1}$ : The microwave reflectance signature of a non-linear (diode) element positioned in the static electric field generated by a nearby radiating source is studied. Moving the diode element through the electric field allows the precise field distribution of the radiating source to be mapped in both the near and far-fields. In practice, this diode is often modulated by some audio tone to improve the measurement sensitivity with the aid of a lock-in amplifier. Electric field perturbations caused by currents induced on the scattering object are related to the microwave reflectance signal observed by the reflectometer through a direct application of the reciprocity theorem.

keywords: Modulated scatterer, electric field distribution of radiating antennas, antenna pattern measurements

These two perspectives of the microwave reflectance problem have remained disjoint in the literature due to the disparate nature of their intended historical

\footnotetext{
${ }^{1}$ The Institute of Electrical and Electronics Engineers (IEEE) has roots dating back 1884 and has been called by different names over the years. Some of the papers we discuss were published under the former governing body known as the Institute of Radio Engineers (IRE). The two IEEE governed journals of interest with regards to the papers mentioned here are IEEE: Antennas and Propagation and IEEE: Microwave Theory \& Techniques (MTT).
} 
applications for the study of charge carrier distributions and antenna pattern measurements. We observe that under several minor conditions, the two formulations can be treated identically by noting the relationship between the modulated conductivity $(\sigma)$ and conduction current $(\mathbf{J})$ as:

$$
\mathbf{J}=\sigma \mathbf{E}
$$

We will pick up this idea in the next chapter where it remains for us to show that the conduction current induced by the illuminating electric field and the liberated charge carriers becomes the source of a new scattered field which perturbs the microwave reflectance.

\subsection{Microwave Reflectance Argument \#1}

We now turn our attention to the first microwave reflectance argument due to modulated sample conductivity as discussed by the physics community in a number of widely cited papers published by Beck et. al. in the Journal of Applied Physics $[11,12,21,22]$. The foremost of these papers treats the problem generally and does in fact touch upon the relation eluded to earlier. However, the primary emphasis in the published papers focuses on the field equivalent transmission line approach using the Fresnel reflection coefficient between a waveguide and load positioned over the mouth of the waveguide.

These arguments usually begin by noting that the physical quantity (sample conductivity) under test will only be gently perturbed so that the change in reflected microwave power may be treated using first-order perturbation theory. 


$$
P=P_{0}+\sigma^{1} P_{1}+\sigma^{2} P_{2}+\cdots+H O T
$$

Since we have control over the strength of the perturbation, we will ensure that it is sufficiently small so that all terms beyond first-order become vanishingly small so that:

$$
P \approx P_{0}+\sigma P_{1}
$$

where $\sigma$ is the sample conductivity perturbing the reflected microwave power. The total microwave power is broken down into two components for analysis. One component is a constant background term that does not vary with time. The other is a small time varying perturbation term representing the quantity of interest.

\subsubsection{Plane Wave Reflection}

To explicitly show the dependence of microwave power on conductivity, consider the propagation of a plane wave along the z-axis in free space and its constituent $\mathbf{E}$ and $\mathbf{H}$ fields. As the wave is constrained to move along one dimension, we may write the fields simply as:

$$
\begin{aligned}
\mathbf{E}(z) & =\hat{\mathbf{x}} E_{0} e^{-j k_{1} z} \\
\mathbf{H}(z) & =\hat{\mathbf{y}} \frac{1}{\eta_{0}} E_{0} e^{-j k z}
\end{aligned}
$$

where $\eta_{0}$ is the free space impedance equal to $377 \Omega$ and $\mathbf{E} \& \mathbf{H}$ are solutions to Maxwell's equations in a homogeneous medium. The wave continues 
to propagate in free space unimpeded until it reaches the interface of a second medium with a different characteristic impedance. Assuming boundary conditions are met so that the tangential components of $E_{x}$ and $H_{y}$ are zero at the interface, wave decomposition carries one portion of the original wave onwards into the lossy medium. It will continue to propagate in the new medium albeit with an exponentially decaying amplitude and rotating phase shift that is a function of the penetration depth into the material. The remainder of original wave reflects off of the surface with an amplitude proportional to the impedance mismatch between the two media. If we define the impedance of the new medium as $\eta$, then the reflected component of the waves will be scaled by a factor:

$$
\Gamma=\frac{\eta-\eta_{0}}{\eta+\eta_{0}}
$$

where $\eta$ is the intrinsic impedance of the lossy semiconductor medium given by:

$$
\eta=\sqrt{\frac{\mu}{\epsilon_{c}}}=\sqrt{\frac{j \omega \mu}{\sigma+j \omega \epsilon}}
$$

In this form, it is clear that the reflection coefficient explicitly depends on the conductivity $\sigma$ wrapped up in the material dependent complex permittivity term of impedance. We now relate the $\mathbf{E}$ and $\mathbf{H}$ terms back to the reflected microwave power. The magnitude of the reflected $\mathbf{E}$ and $\mathbf{H}$ fields are then scaled by the magnitude of the reflection coefficient $|\Gamma|$ and the phase is shifted by $180^{\circ}$ or $\theta=\pi$. Expressing these observations in terms of the fields, we have: 


$$
\begin{aligned}
E_{r} & =\hat{\mathbf{x}} \Gamma E_{0} e^{j k z} \\
H_{r} & =-\hat{\mathbf{y}} \frac{\Gamma}{\eta_{0}} E_{0} e^{j k z}
\end{aligned}
$$

The power transmitted by $\mathbf{E}_{r}$ and $\mathbf{H}_{r}$ can be calculated from the timeaveraged Poynting vector.

$$
P_{r}=-\frac{1}{2} \frac{\left|E_{0}\right|^{2}}{\eta_{0}}|\Gamma|^{2}
$$

The reflected power depends on the square of the electric field intensity, the characteristic impedance of free space, and the impedance mismatch factor $\Gamma$ which is an implicit function of the conductivity $\sigma$. Although we could express the reflected power as an explicit function of the material conductivity, this is rarely done. It is more common to linearize the reflected power about a specific conductivity point so that small changes in sample conductivity correspond to equally small changes in the reflected power. Under these conditions, it is convenient to redefine the reflection coefficient from a ratio of wave reflections to that a ratio of power reflections so that:

$$
\Gamma(\sigma)=P_{r}(\sigma) / P_{i}
$$

\subsubsection{Perturbation of the Microwave Reflectance}

The magnitude of the effect generated by the excess charge carriers is now defined in terms of its explicit effect on reflected power. To study the effect that a change in material conductivity will have on the microwave reflectance 
we perturb the microwave reflection by an amount $\Delta \sigma$. The corresponding change in microwave power is then:

$$
\frac{P(\sigma+\Delta \sigma)-P(\sigma)}{P_{i}}=\frac{\Delta P(\Delta \sigma)}{P_{i}}=\Gamma(\sigma+\Delta \sigma)-\Gamma(\sigma)
$$

We may view the linearized response using a Taylor series approximation about the operating (dark) conductivity point.

$$
\frac{\Delta P(\Delta \sigma)}{P_{i}}=\left[\left(\frac{\delta \Gamma(\sigma)}{\delta \sigma}\right)_{\sigma} \Delta \sigma+\frac{1}{2}\left(\frac{\delta^{2} \Gamma(\sigma)}{\delta \sigma^{2}}\right)_{\sigma}(\Delta \sigma)^{2}+H O T\right]
$$

which is equivalent to the form of Eq. 2.3 for the perturbation equation. Since the perturbation is assumed to be small, i.e. $\Delta \sigma \ll 1$, the higher order terms have little effect on the change in microwave power and can be safely discarded.

$$
\frac{\Delta P}{P_{i}}=\Delta \sigma\left[\frac{\delta \Gamma(\sigma)}{\delta \sigma}\right]_{\sigma}
$$

It is customary in the literature to proceed by defining the change in microwave power relative to the total reflected power rather than the incident power as follows:

$$
\frac{\Delta P}{P(\sigma)}=\frac{1}{\Gamma(\sigma)}\left[\frac{\delta \Gamma(\sigma)}{\delta \sigma}\right]_{\sigma} \Delta \sigma
$$

This form of the reflectance signal is favored in many papers since only 
one microwave diode detector and a single circulator is required to measure the small signal perturbation. Strictly speaking, it is not necessary to know the exact input power level to detect the small change in microwave power. Measurement under these conditions is viable with a sufficient minimum detectable signal level and input power handling capability of the microwave receiver. However, measurement sensitivity is low due to the large signal entering the signal without any information content. We will see an alternate receiver design later in this thesis that removes the unwanted signal content and increases measurement sensitivity. Such modifications are necessary to increase receiver sensitivity as our LED based signal source is several orders of magnitude weaker than the laser based sources typically reported.

The slope of the relative change in reflected power for small conductivity changes is simply:

$$
A=\left[\frac{1}{\Gamma(\sigma)}\right]\left[\frac{\delta \Gamma(\sigma)}{\delta \sigma}\right]_{\sigma}
$$

At this point, we pause to observe that the previous derivations are do not take into account the spatial distributions of the photogenerated carriers. In the case of uniformly distributed photoconductivity across a semiconductor wafer, this argument is sufficient for $\mathrm{QE}$ and lifetime measurements.

\subsubsection{The Effect of Imhomogeneous Conductivity Profiles on Lifetime Mea- surements}

Earlier in the discussion, we had assumed that the reflection was caused by interaction with a homogenous material. Challenges arise when the microwave 
reflectance technique is applied to imhomogenous samples as the carrier distribution throughout the sample can affect the microwave reflectance unevenly. Significant advancements to both the qualitative and quantitative behavior of imhomogenous samples were published by Schöfthaler and Brendel [13] and later in more complete form by Swiatkowski et. al [22]. Qualitatively, we must take extra caution when applying the appropriate scaling factors to the microwave photoconductivity decay to extract lifetime as a solar cell is imhomogeneous by definition. The reason for caution is apparent when one considers the strong electric field setup between the heavily doped emitter layer and lightly doped bulk region. These two regions setup a strong electric field between them which causes carriers to redistribute themselves accordingly. It is reported in [13] that exponential lifetime decay cannot be calculated using average conductivities unless one takes the dependence of reflection on the carrier distribution into account. The new change in reflected power taking the depth dependence into account is modeled as:

$$
\frac{\Delta P}{P(\sigma)}=A \int_{0}^{d} \Delta \sigma(z, t) d z
$$

where $\sigma(z, t)$ represents time-evolving distribution of carriers in the sample.

\section{Experimental Determination of Non-Uniformity Factors in a Solar Cell}

To explore this idea further, experimental methods developed in [22] compare the time-resolved microwave photoconductivity decay signature for two wavelengths at 532nm and $1064 \mathrm{~nm}$. These wavelengths were chosen to excite different depths of a $500 \mu m$ crystalline silicon wafer according to Beer's law. They arrived at an experimental uniformity ratio defined as 


$$
U=\frac{A_{532}}{A_{1064}} \frac{2 I_{1064}}{I_{532}} \beta \epsilon\left[1-\exp \left(\alpha_{1064} d\right)\right]
$$

where A has been redefined to represent the amplitude of the reflectance signal for a particular wavelength, I is the excitation densities used to determine $\mathrm{A}, \alpha$ is the absorption wavelength dependent absorption coefficient, $\beta$ is a factor accounting for multiple reflections between the front and back surfaces, and $\epsilon$ is a scale factor taking into account the different surface reflection coefficients for 532nm and 1064nm photons. Using this notation, we can experimentally test the effect of non-uniform carrier distributions on minority-carrier lifetime for each sample to determine the extent of the scaling factors needed to extract lifetime from the microwave reflectance signature. For pure high quality silicon wafers, it is reported [22] that the uniformity factor $U \approx 1$ as long as the sample is not positioned near a minimum of the probing microwave field. In this situation the carrier decay time is

$$
\tau=\frac{d^{2}}{D \pi^{2}}
$$

where $\mathrm{d}$ is the depth of carriers in the wafer and D is the minority-carrier diffusion constant. However, when the sample is in the vicinity of a microwave probe minimum, the uniformity factor experiences a sharp peaking effect and the blue and red responses of the photogenerated carriers produce significantly different reflection terms. In this work the presence of a PN junction in the material demands that the uniformity factor be examined prior to establishing a scaling factor for the microwave reflectance decay. 


\section{Bias Illumination on Surface Currents in Lifetime Measurements}

Optical bias in the region surrounding the region of interest is shown to have a strong effect on excess charge carrier recombination. In dark measurement conditions without DC bias, carriers generated with an optical probe diffuse to the nonilluminated surface surrounding the optical probe. This results in a reduction of microwave detected recombination since the carriers have diffused away from the probe region. The change in carrier concentration is reported [22] to have the following power law dependence.

$$
\Delta n_{l}(t) \propto t^{-0.5}
$$

The diffusion effect may be eliminated by illuminating both the ROI and surrounding area with a bias light so that surface currents are kept to a minimum and the recombination occurs in the vicinity of maximum microwave probe sensitivity.

\subsubsection{The Effect of Imhomogeneous Conductivity Profiles on QE Measure- ments}

The lifetime arguments just prior arrived at a means of measuring the effect that non-uniform distributions of charge carriers will have on microwave detected minority-carrier lifetime measurements. These errors introduced by nonuniformity occur due to unequal microwave reflection coefficients at the sample surfaces and diffusion errors due to surface currents. These effects are timeresolved over on the order of $\mu s$. A quantum efficiency measurement occuring 
over many generation/decay repetitions is not prone to these effects. We therefore expect the quantum efficiency measurement to maintain integrity as long as the low injection condition is maintained.

\subsubsection{Non-Uniform Microwave Response Due to Phase Shift}

The sensitivity factor $A$ for both quantum efficiency and lifetime does explicitly depend on the depth into the sample such that $A \rightarrow A(z)$. This is obvious if we note that the reflected power is proportional to the square of the electric field which does depend $z$.

$$
P(\sigma) \approx \mathbf{E}^{2}(z)
$$

As the electric field is sinusoidal in nature, the maximum sensitivity is defined where the sample is at phase quadrature where the slope is greatest. Alternatively, the minimum change in sensitivity occurs when the sample is placed near the maximum of the field. We are now in a position to discuss how best to mitigate non-uniformity errors due to sensitivity changes in $\mathrm{E}(\mathrm{z})$. The problem occurs because the relative placement of a sample at a certain height may be large compared to the wavelength of the microwave probe. The problem is mitigated if we select a microwave frequency such that the both the

dimensions and position of the sample in the $\mathbf{E}(\mathrm{z})$ field are small relative to a quarter wavelength.

Since the electric field is strongest at the center of the waveguide and tapers off towards the walls to fit the boundary conditions, a non-uniform measurement response will occur if the optical probe is large and offset from the maximum field. It is essential that the optical probe be kept small relative to 
the dimensions of the waveguide and that it be positioned directly along the center of the boundaries.

\section{Microwave Reflector}

Non-uniform sensitivity issues due to the changing electric field amplitude as a function of sample height and thickness raise the interesting application of introducing microwave reflectors behind the sample to improve sensitivity. This application was systematically reviewed by Schöfthaler and Brendel [13] for high/low and thick/thin semiconductor samples. The results revealed that the highest measurement sensitivity was achieved with a reflector in place and that the strength of the improvement was greatest when the sample is small compared to the wavelength of the source excitation. The authors of the study also noted that there will always exist some small separation distance between the optimal position for sensitivity and optimal position for linearity. Since the microwave probe and optical excitation occur on different sides of the sample in this work, an ITO coated glass sheet of sufficient size may be used as a reflector. Brief tests in this thesis verified the amplitude gain achievable using back reflectors. Measurement data shown in Fig. 6.1 were taken with the aid of a ITO reflector placed directly over the sample. However, ITO covered reflectors were not available with a sufficient size to uniformly cover an entire sample, the use of ITO reflectors was abandoned once a motion control system was added to the system. 


\subsection{Open-Ended Waveguide Measurement}

One paper explicitly focusing on instrumentation design [21] published the design appearing in Figure 2.1. Although there are some variations on the technique, most microwave detected photoconductivity measurements use hardware similar to the one shown to acquire a signal. In this hardware, the sample is illuminated from the backside with a modulated laser pulse while the front side faces the microwave waveguide. The sample is placed on an $x y$ stage allowing the sample to be scanned across the surface of the waveguide. The fundamental or first harmonic of a Nd-YAG laser is used to generate $1064 \mathrm{~nm}$ or 532nm light. Alternatively, some designs use a He-Ne laser at a wavelength of $632.8 \mathrm{~nm}$. The laser beam is chopped with a low frequency chopper wheel and syncronized to a lock-in detector. The DC output signal is then displayed or digitized and sent to a computer for processing. Occasionally, a sample is cut into small pieces and positioned inside the mouth of the waveguide behind an adjustable short circuit to improve sensitivity. It is important that the sam-

ple maintain planarity as the angle will introduce a position dependent phase shift in the microwave signal. 


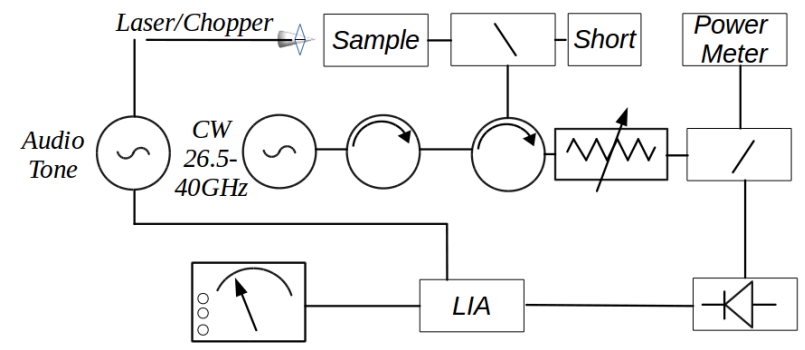

Figure 2.1: Hardware similar to this is configuration is used for all microwave conductivity measurements. The sample is usually positioned over the mouth of an openended waveguide. However, some configurations place the sample inside of the waveguide behind an adjustable short circuit. A circulator differentiates between the incident and reflected power signals. 


\section{The Modulated Scattering Problem}

The detection of photogenerated charge carriers perturbing the microwave reflectance signature of a semiconductor at rest in a static electromagnetic field is nothing more than a special case of the classic modulated scatterer problem. Historically, this scattering technique was created in response to the longstanding problem of accurately characterizing electric field distributions in the vicinity of a radiating source [1]. An antiquated detection method consisting of a reciprocal antenna placed at the point of interest to measure the $S_{21}$ scattering response due to the radiating source yielded precise field patterns under optimal conditions. However, uncontrolled background reflections, antenna cable movement and probe generated field distortions made the experimental setup quite challenging to reproduce. These limitations spurred the development of scattering techniques capable of eliminating or substantially attenuating the severity of these issues [2,23-29]. It is a variant of this scattering technique that plays a central role in the investigation of non-contact quantum efficiency instrumentation and sensing at the core of this thesis.

The purpose of this chapter is to introduce the modulated scatterer technique (MST) using electrically and optically driven modulators, address significant contributions found in the literature, and relate the far-field scattering signals to the waveguide approach discussed earlier. Our trajection will cover MST the quadrature hybrid detection instumentation and begin the scattering 
discussion with a review of dipole antennas. A generalization to aperture antennas is then formed and we introduce the idea of a solar cell as an aperture antenna.

\subsection{Basic Scattering Technique}

A simple circulator based implementation of the monostatic modulated scatterer technique (MST) is illustrated in Figure 3.1. Conceptually, we must perform two operations. First, we need to illuminate of the target with some RF energy. Then we must detect and successfully identify the component of the signal reflected off of a target. Three components are dictated by the problem statement; a source, receiver and target ${ }^{1}$. The remainder of the components are strictly implementation dependent.

1. Modulated Target: A target need only be capable of scattering microwaves illuminating its surface. The general problem considers mechanical, electrical and optically driven modulators. Mechanical modulators may consist of spinning dipoles or moving objects with a recognizable doppler shift. Electrically modulated targets include diodes, raw semiconductor materials or antennas. Optically modulated targets are often small shielded photocells attached to a radiating antenna. Our target consists of a planar solar cell modulator without the aid of an attached antenna.

2. RF Source: The microwave source consists of a continuous-wave (CW) RF oscillator which is used to set up a static field in and around the target.

\footnotetext{
${ }^{1}$ The literature often refers to the modulated object as the probe since this object was placed in the field of a source under test. The target terminology is more familiar in radar system applications.
} 
The microwave source must illuminate the target with a sufficient amount of RF power so that the reflected signal is above the minimum detectable signal level of the receiver.

3. Receiver: The receiver processes the reflected signal caused by perturbation of the static field generated by the CW source. Since the reflected signal is often many orders of magnitude weaker than the unmodulated signal incident on the target, care must be taken to enhance the sensitivity of the receiver to ensure a high probability of detection without comprimizing the accuracy of the measurement by overloading the receiver input with strong unmodulated signals.

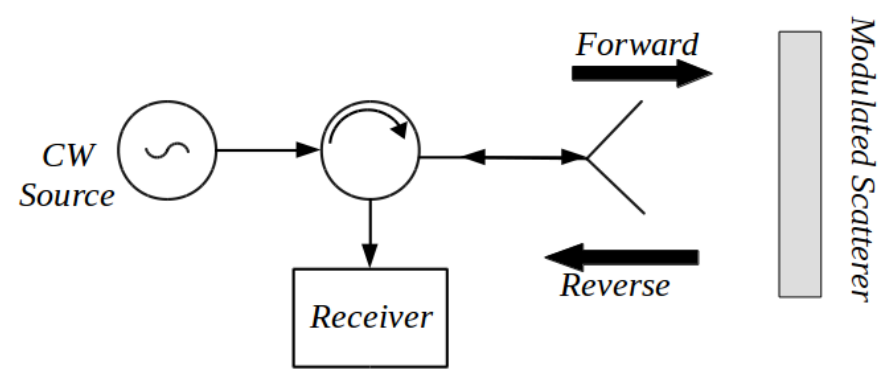

Figure 3.1: Basic monostatic modulated scatterer configuration.

We can see from Figure 3.1 that the basic scattering model is a very straight forward concept. First we illuminate the target; then, we listen to the echo off of the target and probe the signal for information in the receiver. The main advantage of this technique is that no additional wires are required for the scattering target. 


\subsubsection{Classes of Scatterers}

This class of scatterers is referred to as the monostatic scatterer due to the use of a collocated antenna for both the transmit and receive signals. In addition to antenna collocation, the scatterer position is static relative to the radiating field. A second class of scatters deploying additional receiving antennas in which the physical placement of the antenna is distinct from the transmitter position is known as the bistatic or n-static scattering configuration. This configuration serves as the basis for microwave tomography systems which seek to map the surface or subsurface density of an object in 3D. Usually, the tomographic system is placed in an chamber isolating it from external interference while a radiating probe illuminates the object under study. Receive antennas surrounding the object measure the total transmission and reflection coefficients from each transmit receive pair of antennas. The third and final case that we want to introduce is that in which the relative distance between the target and transmitter is changing. One example of such a system is the measurement an accelerating object in a field creating a measureable doppler shift over time. The first two class of scatterers are significant for the desired measurement goals of this thesis.

\subsubsection{Unmodulated Scattering}

Justice and Rumsey demonstrated that an antenna form of the reciprocity theorem that the voltage signal impressed on the terminals of the receiving antenna by the scatterer must be due to the currents flowing on the scatterer in the direction of the impinging $\mathbf{E}$ field. A consequence of this relationship is that it does not matter how those currents first got there, only that they exist. Let's 
consider the experiment that Justice and Rumsey first used to demonstrate the scattering principle. They describe a hybrid junction connected to a microwave transmitter, dummy load, a triple stub tuner, and a receiver as shown in Figure 3.2. The transmitter illuminates the space around the aparratus while the dummy load and triple stub tuner are used to null out all of the background reflections until the receiver reads $0 \mathrm{~V}$.

Now an object is placed in the field so that the nulled terminal of the receiver is unbalanced. The receiver records the magnitude of the unbalnace due to the scattering object. Let's assume that the source is oriented so that the $\mathbf{E}$ field is along the y-axis and that the size of the scatterer is small compared to the wavelength of the plane wave generated by the $\mathbf{E}$ and $\mathbf{H}$ fields. In this case we may treat the scatterer as having any arbitrary shape.

\section{Infinitesimal Dipoles}

Electromagnetic waves impinging on the surface of a dielectric material stretch (or compress) and rotate the atomic dipoles according to the forces applied on them by the external electric field. It is not unreasonable to assume that the dipoles become polarized without time delay upon sensing the electric field ${ }^{2}$. As long as this assumption is valid, the dipoles are constrained to oscillate in phase with the polarizing field. Since we have previously assumed a time-harmonic form of the electromagnetic fields due to the continuous-wave microwave source, these dipoles will oscillate at a frequency of $10.525 \mathrm{GHz}$ in phase with the microwave generator with a dipole moment defined by:

\footnotetext{
${ }^{2} J a c k s o n:$ Section 9.6a-Scattering by Dipoles Induced in Small Scatterers; Griffiths: Chapter 4-Electric Fields in Matter
} 


$$
\mathbf{p}=\alpha \mathbf{E}_{l}
$$

where $\alpha$ is the polarizability factor of the dipole. This statement tells us that the dipole moment is just the magnitude of the electric field scaled by a factor $\alpha$ and oriented along an axis parallel to E. If we assume that the the electromagnetic waves incident on the surface of the scatterer are generated by a current impressed across a pair of antenna terminals, we can relate the source power to the work required to align the dipoles.

$$
i_{0} V=\mathbf{p} \cdot \mathbf{E}
$$

Plugging in the dipole moment into the equation for power we have,

$$
P=A E^{2}
$$

Now, we can see that the moving charges assosiated with the dipoles must also generate a field consistent with a dipole radiator and that the radiated signal is proportional to the square of the electric field parallel to the incident wave and with a rotating phase vector that is twice the incident phase. The claim that the currents impressed on the antenna terminals are in fact related to the fields generated by the induced currents on a nearby scatterer requires some elaboration. Here we have made use of the Lorentz reciprocity theorem to form the equality. When expressed in terms of antennas, the theorem states:

\section{Reciprocity Theorem:}

If an emf is applied to the terminals of an antenna $A$ and the current measured at the terminals of another antenna $B$, then an equal current (in 
both amplitude and phase) will be obtained at the terminals of antenna $A$ if the same emf is applied to the terminals of antenna B. -J. Kraus and R. Marhefka in "Antennas for All Applications" [30].

For Justice and Rumsey, the principle implies that a map of the E fields at each point in space may be observed and recorded by simply positioning the scatter at the point of interest. This technique significantly reduces the errors attibuted to interference from cables and other objects conected to the scattering target making reliable measurements of the field distributions of antennas feasible. However, the system is constrained in three aspects. These main constaints are:

1. Very high isolation is required between the transmitting and receiving electronics to detect the small scattering signal. Justice and Rumsey report that $90 \mathrm{~dB}$ of isolation or greater is required for signal detection.

2. Achieving a $90 \mathrm{~dB}$ isolation performance target requires the use of precision amplitude and phase tuners at each arm of the hybrid coupler. This fine tuning procedure is time consuming and is made obsolete by a shift in temperature and oscillator frequency.

3. The measurement is greatly affected by changes to the background reflectance. An isolation chamber is required for repeatable measurements.

\subsubsection{Electrically Modulated Scatterer}

J. Richmond proposed an adoptation of the scattering technique [2] in the following year that reduced the isolation requirements on the hybrid coupler by 


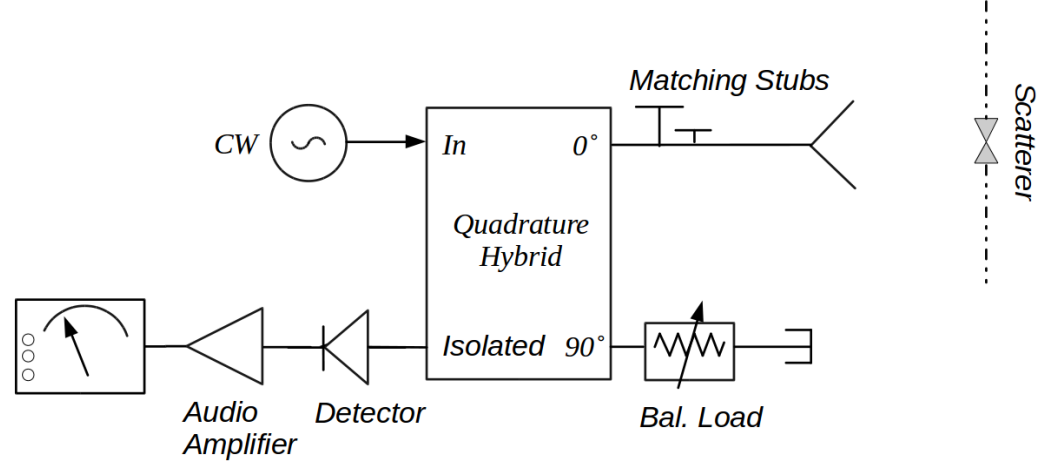

Figure 3.2: Basic monostatic scatterer problem described by Justice and Rumsey [1].

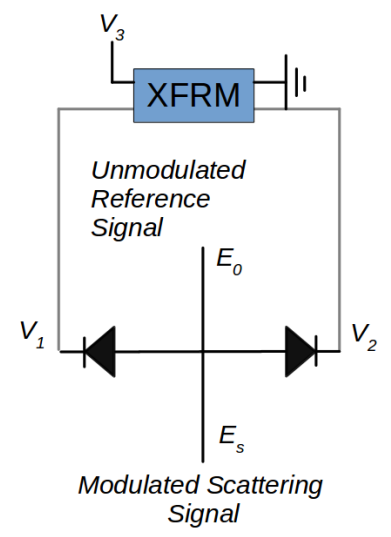

Figure 3.3: The modulated scatterer coherent detection method pioneered by Richmond [2].

more than $50 \mathrm{~dB}$. This new technique applied a modulated signal to the scattering diode itself so that the detected signal was frequency shifted away from the large $1 / \mathrm{f}$ noise present in the measurement. The form of the $V=A \mathbf{E}_{l}^{2}$ found in Justice and Rumsey assumed that there were no time-varying changes to the microwave reflectance. Richmond takes this into accound and neutralizes the effect that these background terms have on system performance by measuring and recording the voltage differential of the scattered signal. The differential measurement senses the background relative to the reference signal derived 
from the isolated and quadrature arms of the hyrid coupler. Combining the two singles removes the common background signal leaving only the smallsignal differential term. The $\mathbf{E}$ field at the two arms of the synchronous detector depends solely on the original illuminating source, the unmodulated scattering field due to external objects and the scattering field due to the modulated scatterer itself.

$$
\begin{aligned}
& \mathbf{E}_{1}=\mathbf{E}_{0} \cos (\omega t)+\mathbf{E}_{u} \cos (\omega t+\beta)+\mathbf{E}_{s} \cos (\omega t+\alpha) m(t) \\
& \mathbf{E}_{2}=\mathbf{E}_{0} \cos (\omega t)-\mathbf{E}_{u} \cos (\omega t+\beta)-\mathbf{E}_{s} \cos (\omega t+\alpha) m(t)
\end{aligned}
$$

where $\mathbf{E}_{0}$ is the unmodulated carrier from the signal generator, $\mathbf{E}_{u}$ is the unmodulated reflection component due to scattering off of nearby obejcts and $\mathbf{E}_{s}$ is the modulated scatterer component. For a square wave modulation term $m(t)$ on $\mathbf{E}_{s}$

$$
m(t)= \begin{cases}1 & \text { if } 0<t<T / 2 \\ 0 & \text { if } T / 2<t<T\end{cases}
$$

The power in the microwave signal is proportional to the square of the electric field. Squaring each term and removing any term not explicitly dependent on $m(t)$ with a bandpass filter we have,

$$
P_{1}=\left[E_{s}^{2} \cos ^{2}(\omega t+\alpha)+2 E_{0} E_{s} \cos \omega t \cos (\omega t+\alpha)+2 E_{u} E_{s} \cos (\omega t+\beta) \cos (\omega t+\alpha)\right] m(t)
$$


$P_{1}=\left[E_{s}^{2} \cos ^{2}(\omega t+\alpha)-2 E_{0} E_{s} \cos \omega t \cos (\omega t+\alpha)+2 E_{u} E_{s} \cos (\omega t+\beta) \cos (\omega t+\alpha)\right] m(t)$

Using a diode detector to remove the microwave frequency and recover a voltage signal proportional to the incident power we have,

$$
\begin{aligned}
V_{1} & =\frac{1}{2} E_{s}^{2}+E_{0} E_{s} \cos (\alpha)+E_{u} E_{s} \cos (\alpha-\beta) \\
V_{2} & =\frac{1}{2} E_{s}^{2}-E_{0} E_{s} \cos (\alpha)+E_{u} E_{s} \cos (\alpha-\beta)
\end{aligned}
$$

Now, the last key step is to measure the differential signal $\Delta V=V_{1}-V_{2}$.

$$
\Delta V=2 E_{0} E_{s} \cos (\alpha)
$$

The consequence of examining the small signal differential change in scattering is that the the system becomes immune to slowly changing variations in background reflectance. The only signal observed by the detector is a constant term at DC proportional to the strength of the illuminating source, the strength of the scattered field and a voltage proportional to the phase angle between the source and the modulated scatterer. We note that specification of the square wave modulation function in this case is actually superfluous. It is sufficient to mark a particular term with modulation when using coherent detection since the modulation falls out in the synchronous detection process.

The differential nature of this technique makes it both immune to slowly varying background reflections and able to detect incredibly weak signals through synchronous detection. Nearly all modulated scattering techniques in use today can trace their roots back to the work performed by Richmond in 1955 . 
The main consequences due to the application of modulation at the field point of interest are:

1. A significant reduction in the isolation requirements of the hybrid coupler. Measurements by Richmond and others showed that the isolation requirement between transmitter and receiver could be reduced from $100 \mathrm{~dB}$ to $45 \mathrm{~dB}$ using the scattering approach.

2. Modulation shifts the tone away from DC providing some immunization against errors introduced by slight changes to the background reflectance.

3. Results valid until unmodulated signal approaches $15 \mathrm{~dB}$ above the maximum scattered signal $E_{s}$ or $35 \mathrm{~dB}$ avove the minimum scattered signal.

The method does have one major drawback however. In order to actuall introduce the modulation at the field point of interest, then a pair of electrical leads still have to be connected to the device and suspended in the field. Any non-linear element may be used as the modulaing component. For this experiment, Richmond suspended a diode in air using a high impedance nylon string. Since the resitivity of the nylon string is not infinite, a voltage signal may still be used to turn the diode on and off at the desired rate.

\subsubsection{Optically Modulated Scatterers}

Practical concerns over the introduction of conductive leads moving in the fields apart from the scatterer is still a problem modulated scatterer technique. Vural and Cheng demonstrated an optically scattered technique using a photocell in $1963[25,26,29]$ that dispenses with the need for any physical connection with the scatterer entirely. These authors appropriately noted that the strength 
of the scattered signal depends on the spectral response, frequency response, sensitivity, and the diode behavior in dark and forward biased conditions. It is precisely this relationship betwen microwave reflectance and the photovoltaic device behavior that we will study in the next few chapters.

1. The photocell modulator removes the requirement for any physical connection between the scatterer and the external world.

2. The authors note the importance of dark and forward illuminated conditions, spectral response, frequency response to the microwave response. The change in background surface conductivity is observed to be of particular importance for the scattering measurement.

Early experiments used CdSe and CdS to conduct experiments with an 20W $\mathrm{W}^{3}$ Zirconium arc lamp. A chopper wheel modulated the light pulses at a frequency of $300 \mathrm{~Hz}$ while a $4 \mathrm{~Hz}$ wide bandwidth coherent detector extracted the signal from the microwave reflectance.

Now that we have had an overview of the most significant scattering concepts, we turn our attention towards the relation between antenna theory and the microwave conductivity arguments presented earlier.

\footnotetext{
${ }^{3}$ The 20 Watt radiated power estimate is based off of type K-300 arc lamp identified in the literature assuming a $70 \%$ optical column efficiency.
} 


\subsection{Microwave Reflectance Argument \#2}

Maxwell's equations written in differential form and applied to time-harmonic fields of the form $\mathbf{E}=\operatorname{Re}\left(\mathbf{E} e^{j \omega t}\right)$ and $\mathbf{H}=\operatorname{Re}\left(\mathbf{H} e^{j \omega t}\right)$ are represented as:

$$
\begin{aligned}
\nabla \times \mathbf{E}+j \omega \mu \mathbf{H} & =-\mathbf{M} \\
\nabla \times \mathbf{H}-j \omega \epsilon \mathbf{E} & =\mathbf{J}_{\mathbf{T}} \\
\nabla \cdot \mathbf{D} & =\rho_{T} \\
\nabla \cdot \mathbf{H} & =0
\end{aligned}
$$

This form of Maxwell's equations is suitable for a study of antenna theory and will be the principle means used to describe the mechanism by which noncontact measurements are made possible. Since the literature on microwave reflectance as applied to the study of solar cells primarily focuses on transmission line theory, we will take some care and start by introducing an equivalent antenna view of the problem using a simple dipole antenna model. Then we will modify the current density function to better approximate the behavior of a solar cell scattering problem and observe the change in field pattern. The application of the Lorentz Reciprocity Theorem in its antenna form is fundamentally important to the idea of a modulated scatterer and solar cell antenna. The proof of the reciprocity theorem is demonstrated in many texts and pa-

pers $[31,32]$. We'll begin by discussing the antenna characteristics of an ideal dipole antenna. 

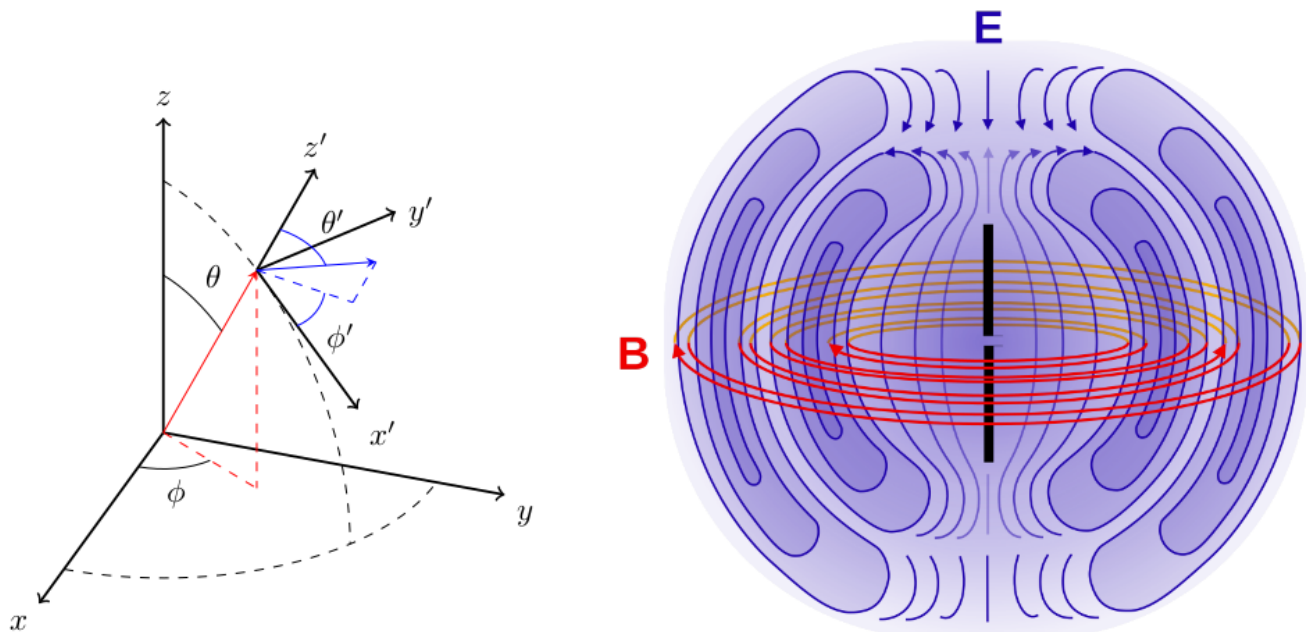

Figure 3.4: We assume that a dipole radiator is used to uniformly illuminate the sample with microwaves. The dipole is oriented on the $\mathrm{z}$ axis and the sample is assumed to lay in the $y-z$ plane.

\subsubsection{Ideal Dipole Antenna}

An ideal dipole or short dipole model is actually a surprisingly useful approximation for many antenna problems since the results can be generalized to any antenna with an appropriate modification to the ideal current carrying element in the magnetic vector potential A. We'll use the classic short dipole model oriented along the z-axis as shown in Figure 3.4 to review the key antenna concepts.

The main challenge in setting up a radiation problem is finding and adding up all of the infintesimal current contributions in an antenna. However, once all of these currents have been determined, it is a relatively straight forward mechanical procedure to uncover the remainder of the antenna characteristics: $\mathbf{E}(r, \theta, \phi), \mathbf{H}(r, \theta, \phi), F(\theta, \phi), \mathbf{S}(r, \theta, \phi)$, directivity $D(\theta, \phi)$, and gain $G(\theta, \phi)$.

The constant of proportionality describing the propagation of waves due to a point source is 


$$
\psi=\frac{e^{-j \beta R}}{4 \pi R}
$$

where $\mathrm{R}$ is the distance separating the field point being probed and the source. If we take a handful of charges and distribute them equitably over an infintesimal line, magnetic vector potential becomes a one dimensional integral dependent on the point source function, the total number of moving charges on the line.

$$
\mathbf{A}=\int_{\Delta z / 2}^{\Delta z / 2} \mu I \frac{e^{-j \beta R}}{4 \pi R} d z
$$

The generalized procedure is as follows assuming that we have determined A for the antenna.

1. Starting from $\mathbf{A}(x, y, z)$, derive $\mathbf{H}=\frac{1}{\mu} \nabla \times \mathbf{A}$.

2. Then convert $\mathbf{H}(x, y, z)$ to $\mathbf{H}(r, \theta, \phi)$

3. Finally derive $\mathbf{E}(r, \theta, \phi)=\frac{1}{j \omega \epsilon} \nabla \times \mathbf{H}$

$$
\begin{aligned}
\mathbf{H} & =\frac{I \Delta z}{4 \pi}\left[\frac{j \beta}{r}+\frac{1}{r^{2}}\right] e^{-j \beta \mathbf{r}} \sin \theta \hat{\phi} \\
\mathbf{E} & =\frac{I \Delta z}{4 \pi}\left[\frac{j \omega \mu}{r}+\sqrt{\frac{\mu}{\epsilon}} \frac{1}{r^{2}}+\frac{1}{j \omega \epsilon r^{3}}\right] e^{-j \beta \mathbf{r}} \cos \theta \hat{\theta} \\
& +\frac{I \Delta z}{2 \pi}\left[\sqrt{\frac{\mu}{\epsilon}} \frac{1}{r^{2}}+\frac{1}{j \omega \epsilon} \frac{1}{r^{3}}\right] e^{-j \beta \mathbf{r}} \cos \theta \hat{r}
\end{aligned}
$$

We end up with seven terms telling us everything we can possibly want to know about both the near and far fields. We have two terms in $\mathbf{H}$ and five terms 
in E. In principle, all that we need to describe the behavior of any antenna is assemble the correct distribution of currents in the A vector. Our goal in this chapter will be to assemble the correct current distribution to describe the radiation pattern of a solar cell antenna. However, before we get there, we need a few more tools.

\subsubsection{Radiansphere}

The radiansphere is an excellent way to visualize the relative distribution that each of the $\mathbf{E}$ and $\mathbf{H}$ terms have in the near and far fields. It is defined as the point where the $1 / r, 1 / r^{2}$ and $1 / r^{3}$ are approximately equal.

$$
r=\frac{\lambda}{2 \pi}
$$

So, we can imagine a little current element sitting in free space surrounded by an imaginary radiansphere of length $r=\lambda / 2 \pi$. If the current element resides entirely inside of the radiansphere, then the information content in the fields is contained almost entirely in the near field $1 / r^{2}, 1 / r^{3}$ terms. Then, if we stretch the current element until it begins to poke outside of the radiansphere, we have shifted the distribution of field components to slightly favor the farfield so that $1 / r$ terms begin to dominate. It is interesting to think about the radiansphere for a $60 \mathrm{~Hz}, 1 \mathrm{~Hz}$ or $0.1 \mathrm{~Hz}$ wave as essentially everything object is in the nearfield of everything else.

\subsubsection{Consideration of Induced Currents in a Dielectric Material}

Let's bring our focus back to the idea of microwaves scattering off of a semiconductor. From an antenna perspective of the world, the incident electromagnetic 
wave has polarized the sample and is causing the atomic dipoles to oscillate at the applied microwave frequency. These moving charges create a scattered field due to the induced currents that are bound to the atomic structure. If we break up the semiconductor up into small slices parallel to the E-plane, we can visualize the behavior of the scatterer as a collection of dipole antennas oscillating in phase with the incident wave. Application of the superposition principle allows us make an effective dipole whose length depends on the strength of the electric field and polarizability factor.

\subsubsection{Modulation of a Microwave Field by Dipole Scatterer}

An atomic argument was made in the previous sections as to the formation of dipole scattering due to bound currents induced by a polarizing field. Now, to connect the modulated scattering concept, we need to effectively transmit an audio tone generated at the scatterer back to the receiver using the microwave carrier wave.

$$
\mathbf{J}=\mathbf{J}_{f}+\mathbf{J}_{b}+\mathbf{J}_{p}
$$

Since we now considered the flow of currents in a semiconductor antenna, the polarization current and free currents come into play. For the materials of interest, the magnetization currents are neglegible. 


$$
\begin{aligned}
\mathbf{A} & =\mu \frac{e^{-j \beta R}}{4 \pi R} \iiint_{\tau} \mathbf{J} e^{j \beta \hat{\mathbf{r}} \cdot \mathbf{r}^{\prime}} d \tau \\
& =\mu \frac{e^{-j \beta R}}{4 \pi R} \int_{z}\left(\mathbf{J}_{f}+\mathbf{J}_{p}\right) e^{j \beta \hat{\mathbf{z}} \cdot \mathbf{z}^{\prime}} d z \\
& =\mu \frac{e^{-j \beta R}}{4 \pi R}\left[\int_{z} \mathbf{J}_{f} e^{j \beta \hat{\mathbf{z}} \cdot \mathbf{z}^{\prime}} d z+\int_{z} \mathbf{J}_{p} e^{j \beta \hat{\mathbf{z}} \cdot \mathbf{z}^{\prime}} d z\right]
\end{aligned}
$$

So the modified magnetic vector potential $A$ for a semiconductor sample illuminated by a microwave source gives yields a microwave reflectance signature which is the superposition of two distinct fields. The first is due to free carriers added during the doping process and the second is due the polarization current which we have no control over.

\subsection{Solar Cell as an Aperture Antenna}

The far-field pattern of an aperture antenna is determined solely by the twodimensional Fourier transform of the $\mathbf{E}$ and $\mathbf{H}$ fields taken across some slice along the radiating face of the antenna. For simplicity, the aperture plane is usually chosen to be the physical aperture and we have assumed normal illumination. Figure 3.5 illustrates a horn antenna on the left over-illuminating a solar cell region in blue. A red square represents the area illuminated by the optical probe. The distribution of charges in the illuminated region may be considered as a uniform distribution aperture problem with electric and magnetic surface currents given by, 


$$
\begin{aligned}
\mathbf{M}_{s} & =-\hat{n} \times \mathbf{E}_{a} \\
\mathbf{J}_{s} & =\hat{n} \times \mathbf{H}_{a}
\end{aligned}
$$

for $\mathbf{M}$ and $\mathbf{J}$ in the aperture. Outside of the illuminated aperture, we consider the remainder of the sample to behave like a screen since the unmodulated reflected power is nulled out through appropriate tuning of the microwave receiver. Later we will see that this is a reasonable approximation since the sample is only placed over the opening of an anechoic chamber. Therefore, the electric and magnetic current densitites are effectively zero outside of the aperture.

$$
\mathbf{M}_{s} \simeq \mathbf{J}_{s} \simeq 0
$$

Under these circumstances the radiation pattern is determined by the two dimensional Fourier integral of the aperture area with length a and width $b$. The radiation pattern results may be found in any number of texts [30,33],

$$
F(\theta, \phi)=\frac{\sin X}{X} \frac{\sin Y}{Y}
$$

where,

$$
X=\frac{a}{\lambda} \sin \theta \sin \phi
$$

and, 


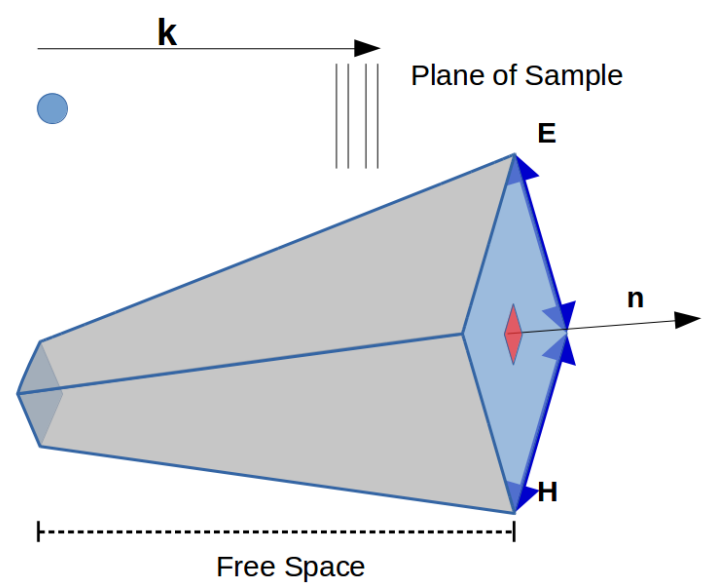

Illuminated Area On Sample

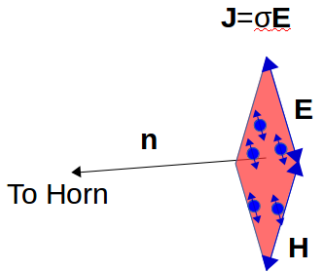

Effective Aperture

Figure 3.5: The effective aperture of the uniformly illuminated solar cell is the area of the optical probe. Librated charge carriers are free to oscillate in phase with the incident $\mathbf{E}$ field. A new current is induced on the sample due to both the incident electric field and liberated charge carriers.

$$
Y=\frac{b}{\lambda} \sin \theta \sin \phi
$$

These results assume a coordinate system with the $\mathrm{z}$ axis oriented normal to the aperture plane. The normalized electric field components are given by,

$$
\mathbf{E}(\theta, \phi)=\left|\mathbf{E}_{0}\right|\left(\frac{1+\cos \theta}{2}\right)|F(\theta, \phi)|
$$

We want to take a moment to emphasize the importance that the illuminated solar cell radiates a scattering field similar to that of an aperture antenna. We can use this fact in our later measurements to setup a custom illumination geometry on the surface of the solar cell. For a high resolution mapping measurement, the illumination and radiation aperture should be kept small. The optical probe size is easily adjustable through a focusing lens. The microwave aperture at the aperture plane can be adjusted through various slots in an RF absorber placed between the sample and microwave antenna. For a fast inline 
measurement, the full sample can be illuminated from a horn antenna in the far field. Different wavelengths of light incident on the front surface of the sample would contribute to the average microwave reflectance for the entire sample. 


\section{Performance of a Solar Cell}

The purpose of this chapter is to introduce the standard contact style quantum efficiency measurement technique as a baseline for comparison with the new non-contact microwave detected quantum efficiency measurement. We will not discuss the fundamental physics behind the absorption of light, generation of free carriers or recombination effects as the subject is broad and our discussion of instrumentation and sensing techniques does not need to delve into the subject matter at that detail. We will however qualitatively describe many defect characteristics which will show up in the quantum efficiency measurement.

\subsection{Spectral Binning of Solar Cells}

Figure 4.1 illustrates both ideal and non-ideal quantum efficiency curves. In an idealized device, all photons of sufficient energy should transfer its energy into the cell and liberate a charge carrier capable of being swept out of the circuit to do work. Here, the efficiency is $100 \%$. Photons without sufficient energy are not capable of liberating carriers. Therefore the efficiency is $0 \%$. The silicon solar cell market is well established with over 40 years of development work and virtually all mono-crystalline silicon solar cell manufacturers produce cells with over $90 \%$ efficient cells in the visible spectrum. The second graph in Figure 4.1 highlights three regions of interest for solar cell efficiency improvements. While a real solar cell device is capable of generating electricity from short wavelength photons, many of the incident photons are reflected 
off of the surface and do not have a change to liberate charge carriers. Therefore the overall efficiency is lower due to surface reflection. Another cause of lower short wavelength $\mathrm{QE}$ is that the surface represents large discontinuity in the crystalline structure leaving many dangling bonds. Free carriers generated near the surface are more likely to recombine with one of the dangling bonds before it can get swept out into an external circuit to do work. The surface 'traps' the carriers lowering the QE.

Level reduction across Region B can indicate an overall resistance problem between the low impedance metallized contacts and/or surface fingers. The insertion loss of the contacts limit the overall efficiency with which photons can be converted into useable electricity.

Low quantum efficiency in Region C can once again be caused by rear surface traps. Of these three regions, Region A and Region C near the front and back surfaces of the solar cell can yield the most significant improvement in efficiency.

Quantum efficiency is an excellent technique with which to monitor the front and rear surface defects limiting the performance of the solar cell device. There is a competing factor for which must be considered during the manufacturing process related to module engineers. Solar cell modules are comprised of a series-parallel combination of individual solar cells. As all cells in a series connection must pass the same amount of current, the performance of the entire chain is limited by the worst performing cell. Thus very high performing cells rolling off the manufacturing cells are ultimately no better off than the worst performing cell in the line if they are placed in series within a same module. Therefore in order to improve module efficiency and total power 


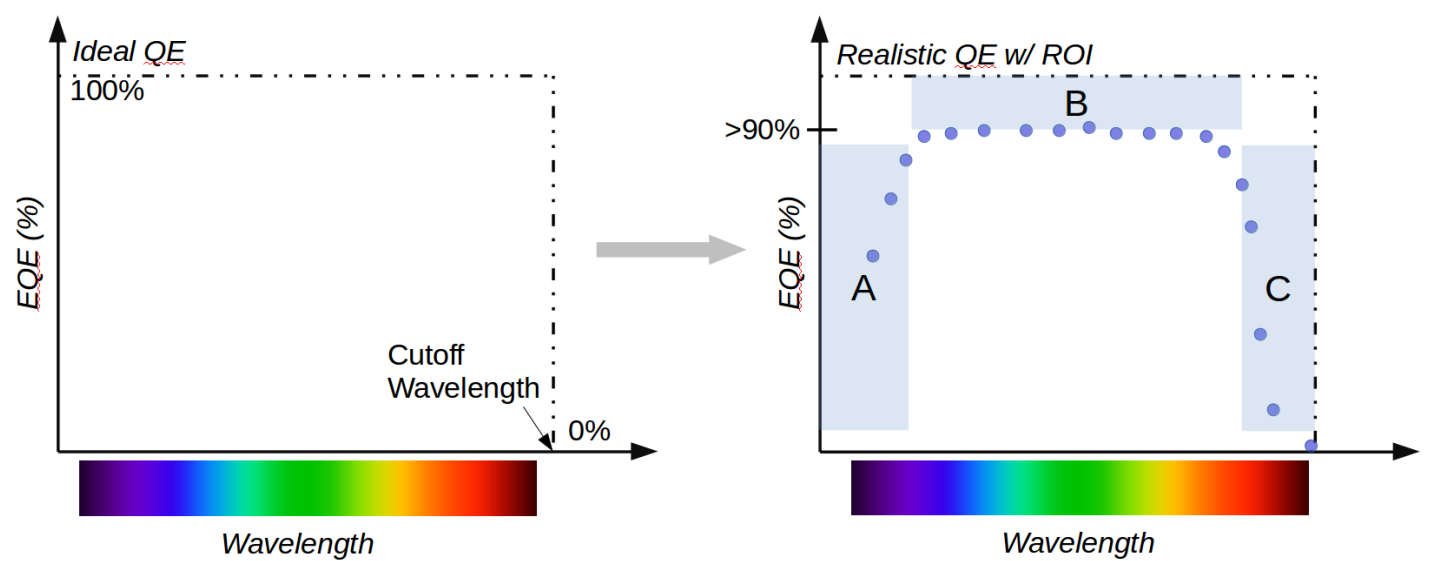

Figure 4.1: The quantum efficiency curve is a single variable function of the incident photon energy. Ideally, any photon with sufficient energy (i.e. $>1.12 \mathrm{eV}$ in silicon) to generate a charge-hole pair should be available to do work if an external circuit is connected. Sample defects preventing the carriers from being extracted will lower the overall quantum efficiency.

generation, the cells must be binned according their individual current output before being placed into a module. Therefore the spectral binning of solar cells is a very important factor in the power generation bottom line.

\subsection{Standard QE Measurement Technique}

The measurement determining solar cell quantum efficiency is routinely performed in the dark with the cell held at a fixed short-circuit bias condition.

A standard quantum efficiency measurement is defined by the ratio of charge carriers liberated to incident photons at each wavelength of light. Convention states that the measurement should occur while the solar cell is held in a short-circuit bias condition to extract photogenerated charge carriers from the device. Typically, a high power Xenon arc lamp is used as a broad spectrum light source ${ }^{1}$. The radiated light is passed through any number of necessary

\footnotetext{
${ }^{1}$ LED technology is understood to have reached sufficient maturity in industry to warrant
} 
filters until the desired spectrum is produced at the other side. Typically a bandpass filter wheel is sufficient to generate light with $20 \mathrm{~nm}$ resolution.

The short-circuit current is then extracted from the device fed into a transimpedance amplifier for additional analog signal processing using a lock-in amplifier. The integration time for the lock-in amplifier is typically between $30 \mathrm{~s}-60 \mathrm{~s}$. The total time duration for the measurement for all wavelengths is approximately 10-20min per measurement cite.

\subsubsection{Contact QE Curve Analysis}

We noted in Figure 4.1 that the ideal QE curve will be a box with perfect collection and conversion efficiency for all photons with energy greater than the bandgap energy of the semiconductor material. However, a number of limitations prevent this from ever occuring and $\mathrm{QE}$ is an excellent tool that can help identify which effects dominate in a particular sample.

\section{- Region A:}

Emitter diffusion length: Low emitter diffusion length will reduce quantum efficiency in the blue response.

Front surface recombination: Emitter damage (high surface recombination) will show up as a lower blue response on the QE curve.

AR coating quality: A poor blue response could also be attributed to poor AR coatings limiting the amount of light reaching the device.

Optical Bias: Surface traps are studied using low intensity dc light

the design and utilization of an entirely solid state light source. However, this solid state technology is still not widely adopted despite the numerous advantages over lamp technology [34]. 
bias sources to fill traps allowing more photogenerated carriers a higher chance to escape the cell and do work. Plots of QE vs bias are also used to determine the behavior of the device along different points of its $\mathrm{JV}$ curve.

- Region B:

Contact resistance: The entire QE curve will be dragged lower due to higher insertion loss with the external circuit. This is not to be confused with surface sheet resistance.

Sheet resistance: The surface sheet resistance of the solar cell is typically studied by analyzing $J_{s c}$ maps of the device. The total current contribution for all wavelengths can show regions of high impedance where current has trouble making it out of the circuit.

Shunt resistance: Low shunt resistance will lower the QE response for all wavelengths as well since carriers are less likely to make it to the contacts at the edge of the sample.

Broken fingers or busbars: Maps of current distribution will show issues with broken contacts inhibiting current flow.

- Region C:

Bulk diffusion length: Low bulk diffusion length will reduce quantum efficiency in the red response.

Back surface recombination: Damage occuring on the back surface of the sample can be recognized by a higher recombination rate or lower red response on the QE curve. 
Rear reflector: Many cells have a reflector layer at the back to give low energy photons the opportunity to pass through the solar cell one more time. Maps of the rear reflector are useful for determining long wavelength performance. 


\section{Systems Level View}

This chapter offers a systems level view of all of the major hardware components that are brought together to make the QE measurement possible. These major subcomponents include the LED light engine, optical column, RF isolation chamber, microwave receiver, motion control system, analog signal processing electronics, one microwave antenna and one microwave lens. Of these components, only the RF isolation chamber, microwave receiver, HS USB data acquisition system (hardware, firmware \& software) and RF antennas were developed from concept to implementation. The LED light engine, optical column and optical reference detectors were assembled using existing hardware and designs published by Ehrenberg et al in [20] were used as a reference for the NIR lens application testing.

A non-trivial level of supporting firmware and software was also developed to communicate with the data acquisition system and configure bidirectional digital interfaces with each hardware subassembly. Hardware components requiring the development of supporting firmware and/or software include the LED light engine, HS DAQ and motion control system. Software components to perform the $\mathrm{QE}$ and lifetime measurement were developed to generate mapping recipes, allocate an LED spectrum, calibrate hardware, send/receive commands, run a QE or lifetime measurement, save data or load data, and display data. Flow diagrams and process charts illustrating the interaction of software and hardware components are discussed in detail. Core routines required 
Table of System Components or Evaluation Data References

\begin{tabular}{|c|c|c|}
\hline System Level Component & Description & Figure \\
\hline Microwave Reflectometer & a) RF system schematic & 5.2 \\
\hline \multirow[t]{3}{*}{ Solid State Light Engine } & a) LED spectrum, load lvl req' & 5.3 \\
\hline & b) Light engine Characterization & Table 5.2 \\
\hline & c) Optical column & 5.4 \\
\hline Motion Controller & $\begin{array}{l}\text { a) Est. Measurement Time vs. Map } \\
\text { resolution }\end{array}$ & 5.3 \\
\hline \multirow[t]{2}{*}{ Analog Electronics } & $\begin{array}{l}\text { a) High speed USB data acquisition } \\
\text { system }\end{array}$ & 5.5 \\
\hline & $\begin{array}{l}\text { b) Measurement stability and temper- } \\
\text { ature drift }\end{array}$ & $5.16,5.17$ \\
\hline \multirow[t]{2}{*}{ Antenna Fabrication } & $\begin{array}{l}\text { a) Measured } \Omega \text {, surface characteriza- } \\
\text { tion }\end{array}$ & 5.4 \\
\hline & b) Antenna $S_{11}$, beam pattern & $5.9,5.10$ \\
\hline \multirow[t]{2}{*}{ Software Components } & a) Standard QE map routine & 5.14 \\
\hline & b) Standard subprocesses & 5.15 \\
\hline
\end{tabular}

Table 5.1: Summary of system components for non-contact microwave QE measurement instrumentation.

specifically for system calibration, data extraction, map recipe generation, and the configuration of spectral recipes are provided in Appendix A. In the following sections, we discuss the necessary hardware and software components that have been either assembled or developed in support of the non-contact measurement system.

\subsection{Microwave Reflectometer}

The fundamental challenge inherent in the design of any front-end RF receiver is the ability to detect small signals masked by the presence of strong RF interference and background radiation that is usually many orders of magnitude larger than the signal of interest. Selective filtering and low-noise amplification are applied at the front-end of the receiver close to the antenna to reject 


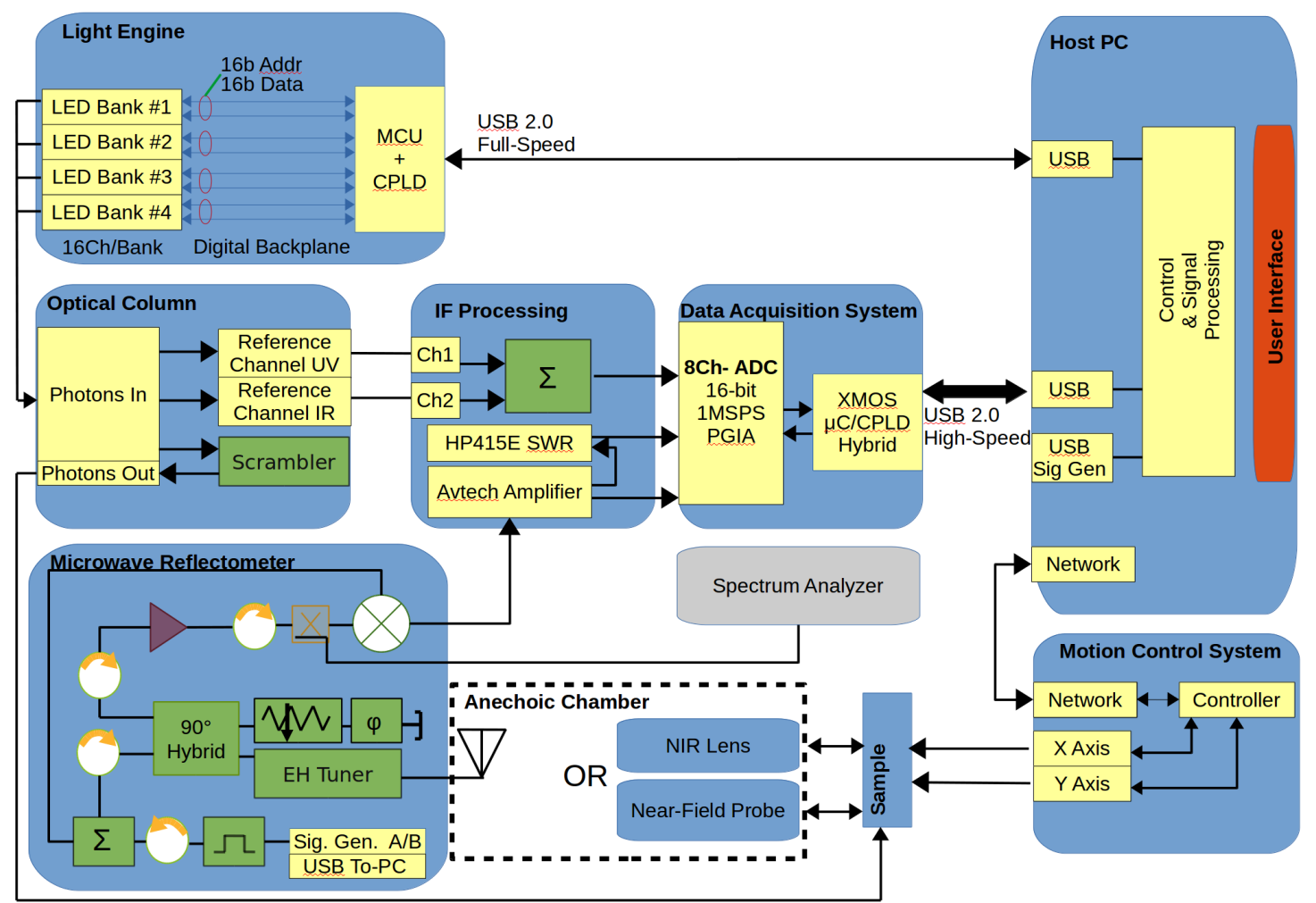

Figure 5.1: System Diagram: This high level view of the measurement system illustrates all of the major components used to acquire the QE/lifetime data.

out-of-band signals while increasing the sensitivity to the remaining signals in the passband. ${ }^{1}$ Unfortunately, competing requirements between the acceptable insertion loss, filter $Q$, and attenuation strength usually require multiple stages of filtering to be implemented throughout the receiver architecture. The level of unwanted signals that do make it past the filter and into the receiver place additional work on the front-end low-noise amplifier (LNA) which must be

\footnotetext{
${ }^{1}$ It is interesting to think how we now consider the urban city dwelling to be a 'hostile' RF environment because of the constraints placed on transceivers while operating in the presence of many other radiating devices. One can imagine how difficult the design constraints must be in the field of electronic warfare where the airwaves are intentionally flooded with signals designed to confuse or damage other RF receivers in the area.
} 
able to handle the RF level without introducing distortion and intermodulation terms.

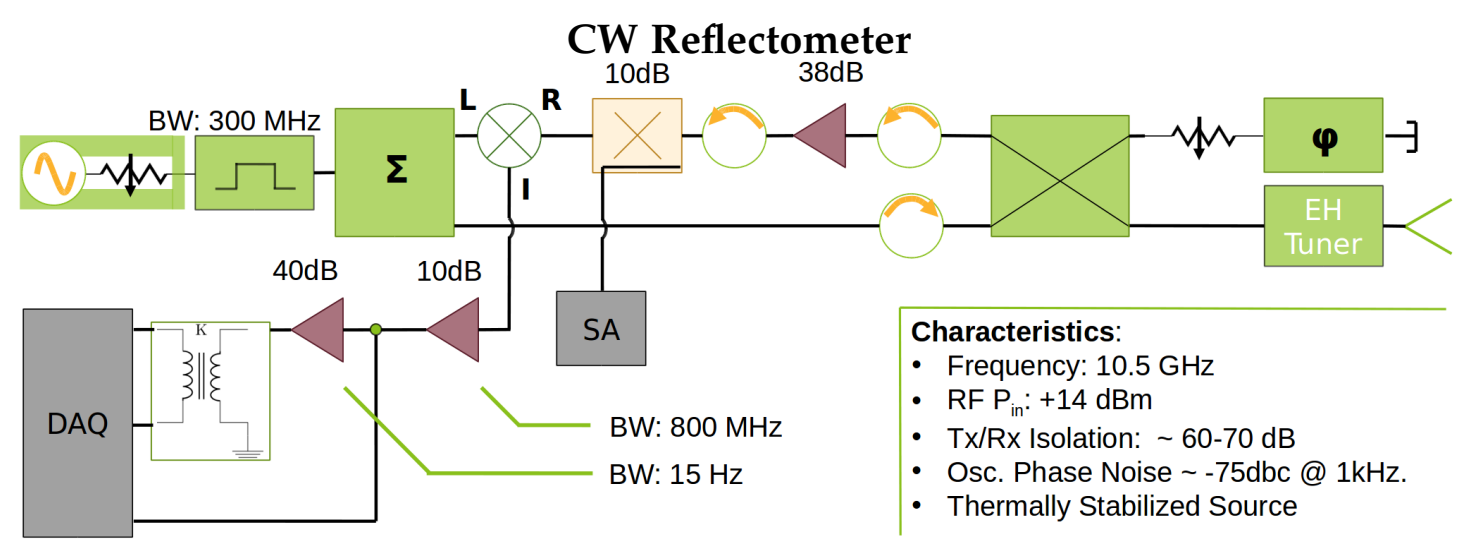

Figure 5.2: Microwave Receiver: A block diagram of the $10.5 \mathrm{GHz}$ homodyning receiver used to both illuminate the solar cell with microwave energy and demodulate the reflected AM tone from the optically modulated solar cell. A digitally controlled source sets both amplitude and frequency

The homodyning receiver mixes two signals of the same frequency to extract baseband information. This technique is simpler than the typical heterodyning receiver due to doing the downconversion in a single step. However this means that the front end RF stage has to do all of the RF signal processing work which. This introduces three challenges. The first challenge is to achieve sufficient signal selectivity. In order to select a specific channel frequency, receiver uses a bandpass filter at the antenna in order to filter out all other signals outside of the passband of interest. A $300 \mathrm{MHz}$ filter (2.8\% Bandwidth) filter is used at the front end to attenuate out of band harmonic components of the oscillator. The entire system microwave system is placed inside of a anechoic chamber to filter out external signals. In this case, the narrow band filter in the receiving arm of the reflectometer is less critical while the system is placed inside of the chamber. It was removed in the hardware configuration used to 
collect data.

The key feature of this reflectometer design for our purposes is a high (minmium 55dB) degree of carrier cancellation in the receiving arm across in a 1 $\mathrm{MHz}$ bandwidth. The impedance tuner, phase and amplitude trim as well as frequency control of the oscillator make achieving greater than $80 \mathrm{~dB}$ possible over a narrower bandwidth without too much effort. We use the high carrier rejection allowed by the hybrid design to place a $38 \mathrm{~dB}$ LNA in the microwave path to amplify the weak AM modulated tone.

Mixing the reflected signal with a reference portion of the oscillator output prior to AM modulation allows for direct conversion back to baseband with a single RF mixer. The simplicity and relatively low cost X-Band reflectometer is

a key feature of the design. Later in this chapter, we show that the oscillator choice is essential to the success of the design and how component costs can be reduced through the use of custom 3D printed RF components.

\subsection{Hardware Implementation}

\subsubsection{Light Engine}

A solid-state light engine covering the entire silicon solar cell spectrum from 365-1200nm highlights the recent advancements in LED technology. Improved spectral reliability, amplitude stability and source longevity contribute to the repeatability performance of the system. This light engine is comprised of 64 unique LED channels modulated serially in turn. Figure 5.3 illustrates a typical LED based light source spectrum. The distribution of available wavelengths and power generation capability make load balancing necessary. Groups of the weakest LEDs are combined into a single measurement to boost signal strength 
for a balanced signal-to-noise ratio across the entire spectrum. Individual optical filters for each LED define the spectral resolution of each LED channel. We characterize the stability of the light engine by recording the reference channel amplitude over a 1.2 hour measurement period using a custom 16-bit data acquisition system.

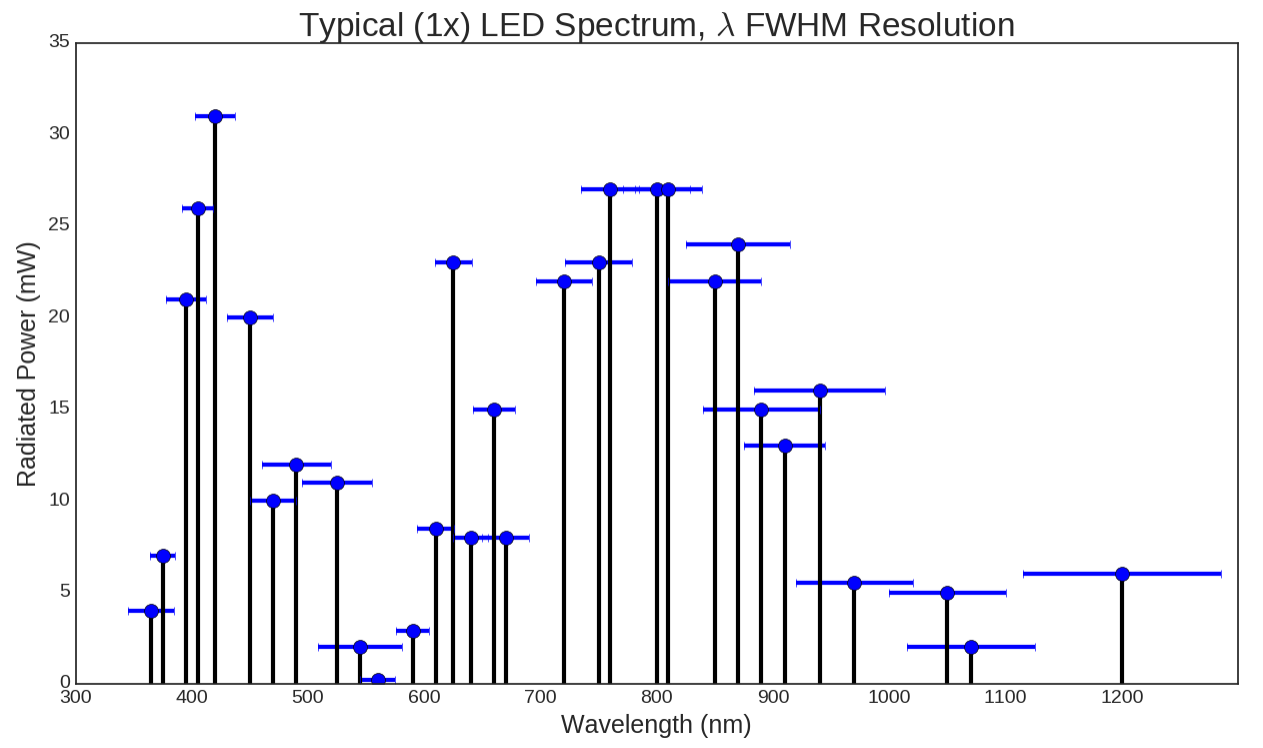

Figure 5.3: Spectral Resolution: A typical LED spectrum showing available wavelengths before optical insertion losses in the fiber optic and scrambler. Power balancing is required to improve detection efficiency on weak signals as well as audio frequency dynamic range requirements. The light engine is designed to support repetitive LED channels to boost power output of the weakest LEDs. Convolution with a typical solar cell quantum efficiency curve indicates that optical power should be balanced around $20 \mathrm{~mW}$ to maintain constant signal-to-noise ratio across the spectrum for the current setup. 
Single Point Repeatability Performance (250x, 1.2Hrs)

\begin{tabular}{lll}
\hline Wavelength $(\mathrm{nm})$ & Reference Channel SNR & SNR, Remove $(>2 \sigma)$ \\
\hline & & \\
365 & 77 & 77 \\
405 & 516 & 544 \\
420 & 1377 & 1431 \\
450 & 86 & 134 \\
490 & 109 & 161 \\
525 & 125 & 189 \\
620 & 119 & 177 \\
690 & 57 & 106 \\
770 & 81 & 132 \\
810 & 104 & 162 \\
870 & 89 & 147 \\
910 & 97 & 156 \\
970 & 94 & 137 \\
1050 & 171 & 183 \\
1070 & 97 & 116 \\
1100 & 43 & 43 \\
\hline
\end{tabular}

Table 5.2: Summary of measurement repeatability with stacked weak leds. 


\subsubsection{Optical Column}

A 64-channel fiber optic bundle is used to couple the individual LEDs into a single core. The optical column collects the light from the fiber optic and homogenizes the individual signals to provide uniform power distribution across the sample beam width. An aperture is used to limit the beam width at the cost of higher optical insertion loss. The signal is collimated and a pick-off mirror monitors the incident power intensity. The combination of the solid state light source and reference power monitoring provides high measurement stability.

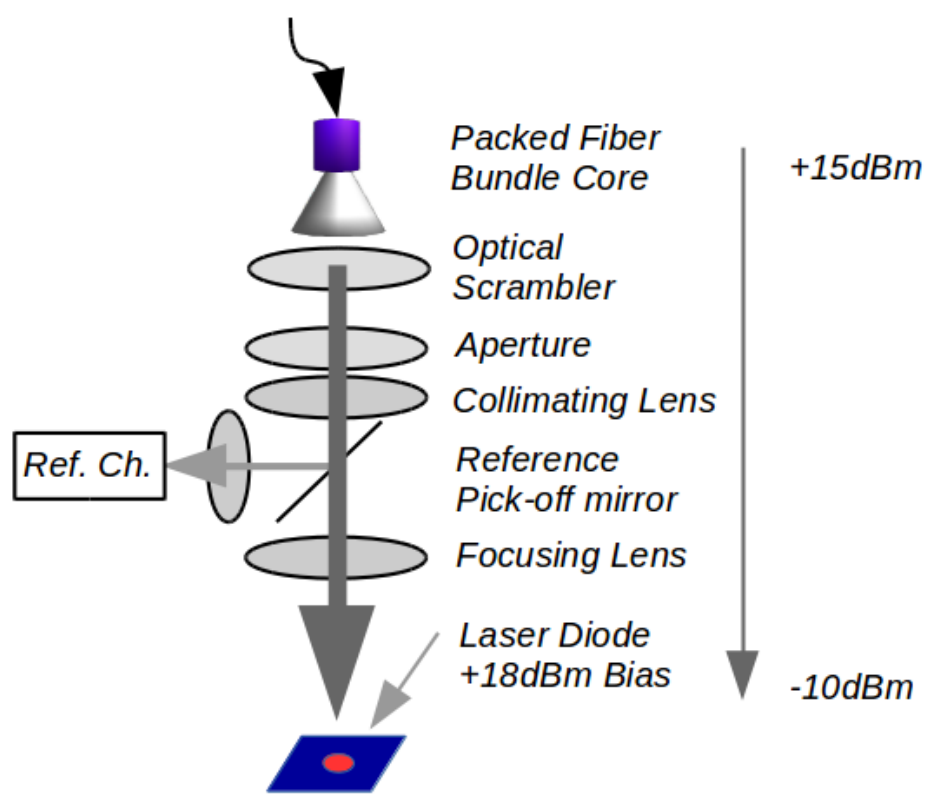

Figure 5.4: Light Engine: 64 independent solid state light sources are packed into a fiber optic bundle and mixed inside of an optical scrambler to homogenize the individual channels. The scrambler is coupled to a collimating lens via an adjustable spot size aperture. Approximately $4 \%$ of the collimated signal is picked-off as a reference signal to monitor the incident number of photons hitting the sample. Finally, an output lens focuses the light back into a spot at the sample plane. 


\subsubsection{Supporting Baseband Electronics}

Two baseband signal processing configurations are possible depending on the bandwidth requirements of the quantum efficiency and lifetime measurements respectively. Quantum efficiency measurements are not time-dependent and may either be integrated with the application of a synchronous detection method (exempli gratia a lock-in amplifier) or passed through a tuned narrowband amplifier to reduce bandwidth and increase signal sensitivity to a particular frequency.

Unlike QE data, lifetime data is time-resolved and actually imposes significant performance requirements on both the data acquisition system and measurement signal-to-noise ratio. In this case, the estimated duration of a minority-carrier in the bulk material is on the order of $50-100 \mu s$. The surface recombination rate will be significantly higher still. We estimate that a $1-2 \mu \mathrm{s}$ sampling rate is sufficient to capture both surface and bulk recombination effects with high enough resolution for the Levenberg-Marquardt algorithm implemented in the signal processing software block. This $1 \mathrm{MHz}$ bandwidth also implies that the microwave receiver noise floor must go up by at least six orders of magnitude.

In both the QE and lifetime measurements, high amplitude sensitivity is required to detect small changes to the number of photogenerated carriers. A 16-bit reconfigurable front-end analog-to-digital converter was chosen to accommodate a number of different signal intensities depending on the specific application. The standard configuration used in this thesis assumes a maximum input signal of $\pm 5 \mathrm{~V}$ with a minimum signal sensitivity of $76 \mu \mathrm{V}$ but is capable of detecting $20 \mu \mathrm{V}$ on a $1 \mathrm{~V}$ maximum signal. In any case, the data 


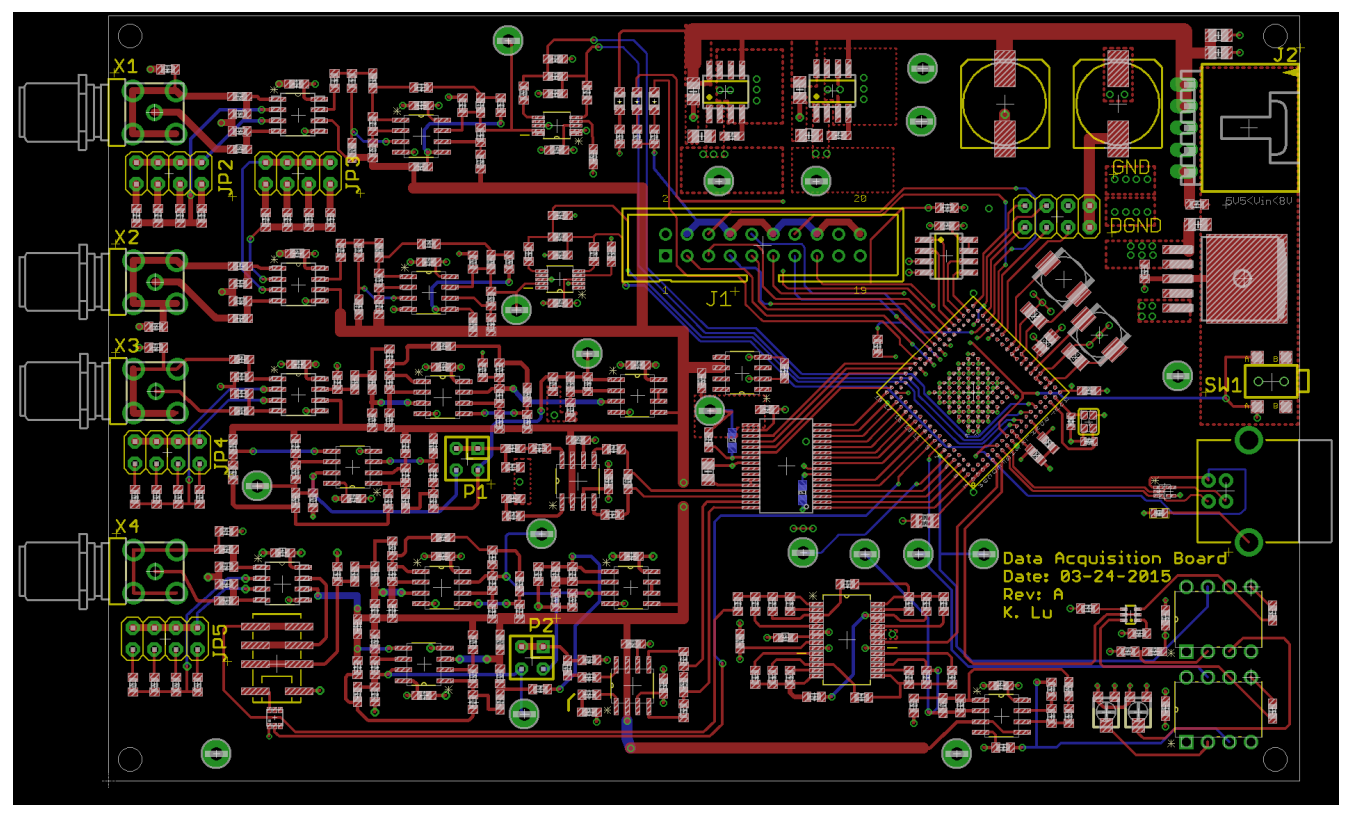

Figure 5.5: DAQ Electronics: The first revision of electronics was designed to accommodate four channels with a sampling rate of 1MSPS per channel. Data is streamed out over high speed USB protocol at $480 \mathrm{Mbit} / \mathrm{s}$. Measurement of a few solar cell samples experimentally demonstrated that the sampling rate requirement could be relaxed slightly. A second revision of hardware using the same processor in a leaded package and with a more versatile ADC was also fabricated.

acquisition system is designed to survive input signal levels of $\pm 15 \mathrm{~V}$ without damage for any input configuration.

Firmware between the data acquisition hardware and software PC controller was implemented in the XC hybrid language developed for XMOS realtime microcontrollers. The XC combines the software flexibility of a language like $\mathrm{C} / \mathrm{C}++$ with a hardware description language such as Verilog. The main advantage of such a system is that the hardware designer may implement software features for data processing in $\mathrm{C}$ and distribute the computational power across 16 different parallel cores. Since every task is implemented in parallel with communication between tasks restricted to specific clock cycles via well defined interfaces, the use of interrupts is eliminated in this architecture. The 
processor core operates at a $500 \mathrm{MHz}$ clock rate while each external single bit port is limited to a $100 \mathrm{MHz}$ clock cycle. This system is perfectly capable of performing DSP tasks in real time. However, since the exact level and integrity of the reflected signal was not known at the time of the development, the microcontroller is configured to simply buffer all data and stream the information out over a high-speed USB 2.0 protocol capable of delivering $480 \mathrm{Mb} / \mathrm{s}$ on demand.

\subsubsection{Stage Control \& Map Generation}

A motion control subsystem implemented over networked Ethernet protocol is used to control the xy stage for mapped lifetime and QE measurements. Maps recipes are generated prior to the start of a measurement per the user specified step size and cell dimensions. The stage is arbitrarily software limited to 100 $\mu m$ step size which, for a typical cell dimension of $156 \mathrm{~mm} \times 156 \mathrm{~mm}$, yields a $2 \mathrm{MP}$ image. This resolution is more than sufficient to detect common surface defects such as wafer scratches. In Table 5.3, we estimate the measurement duration for a variety of map sizes. These estimates assume a single wavelength probe is fired at each measurement site with a 1s measurement period. The measurement time scales linearly with the desired wavelength resolution. Therefore it is necessary to adjust both the spatial resolution and wavelength resolution if a certain minimum throughput is required.

It is possible to significantly increase measurement throughput by stacking up the unique LED sources in Fourier space and measuring all channels simultaneously. The current (and therefore scattering field) contribution from each 


\section{Map Generator}

\begin{tabular}{ccc}
\hline Step Size (um) & \# Pixels $(k P)$ & Est. Measurement Time (Hr) \\
\hline & & \\
5000 & 1 & 2.7 \\
1000 & 24 & 6.7 \\
500 & 100 & 28 \\
100 & 2400 & 670
\end{tabular}

Table 5.3: Summary of map resolution and timing generated from sample size and desired resolution. These values assume a standard $156 \mathrm{~mm} \times 156 \mathrm{~mm}$ silicon sample and 17 unique wavelengths.

can be extracted from the modulated reflectance signal using a Fourier Transform and picking off the components of the signal at each of the known modulation frequencies. However, this technique can potentially introduce complications if the intermodulation spectrum begins to overlap adjacent wavelength channels. The nonlinear nature of the device under test implies that frequency mixing will exist. If the desired signal measured by the system is already weak for any reason, then the intermodulation term will appear as an erroneous term in the measurement. The error terms must be quantified at each point along the JV curve so that a new calibration curve can remove the offset errors for each wavelength.

\subsubsection{Microwave Lens \& Antenna Fabrication}

We have successfully fabricated and deployed two 3D printed microwave antenna components in the final assembly of the non-contact measurement system presented in this thesis. The purpose of this section is to first describe 

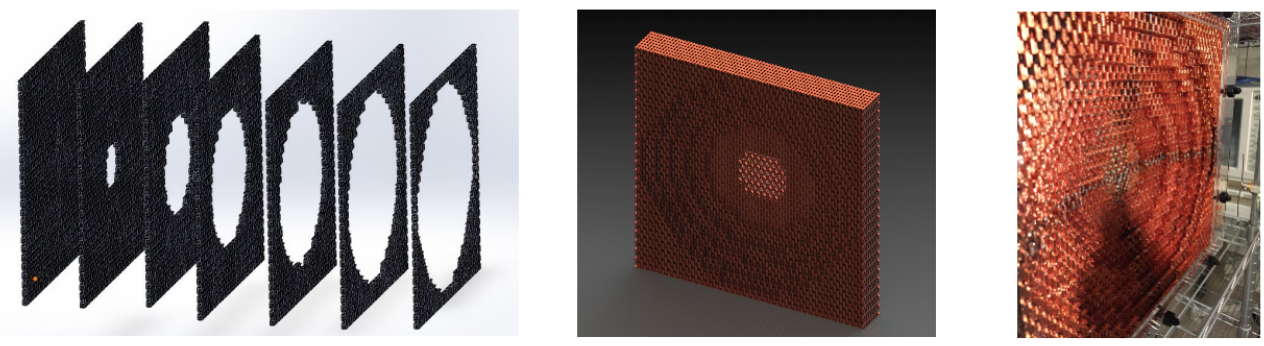

Figure 5.6: Microwave Lens: The final microwave lens was fabricated using a Form $1+3 \mathrm{D}$ printer and homebrew electroplating tank [?].

the low-cost fabrication methods in detail and then provide evidence supporting their operational viability as a high quality alternative to expensive microwave off-the-self antennas. The first component that we shall discuss is the microwave lens shown in Figure 5.6 first published by Ehrenberg et al. [20] in 2012. Unlike many 2D printed circuit board NIR lens designs, this lens is designed as a 3D structure of evenly spaced unit cell layers defined by the hyperbolic surface $S(r)$ in Equation 5.1:

$$
(S(r))^{2}=(f-r)^{2}-(f-n \cdot r)^{2}
$$

where $f$ is the focal length of the lens, $\mathrm{n}$ is the frequency dependent negative index of refraction, and $r^{2}=x^{2}+z^{2}$ is the negative area defining the strength of lens concavity. The authors go on to demonstrate Ansoft HFSS field simulations of the 3D printed lens followed by supporting data measured in the laboratory. The lens was printed on a Connex 500 Object 3D printer and subsequently metalized with $4 \mu m$ of copper in low vacuum using a sputtering process.

Several concerns over the potential for thermal damage during the coating 


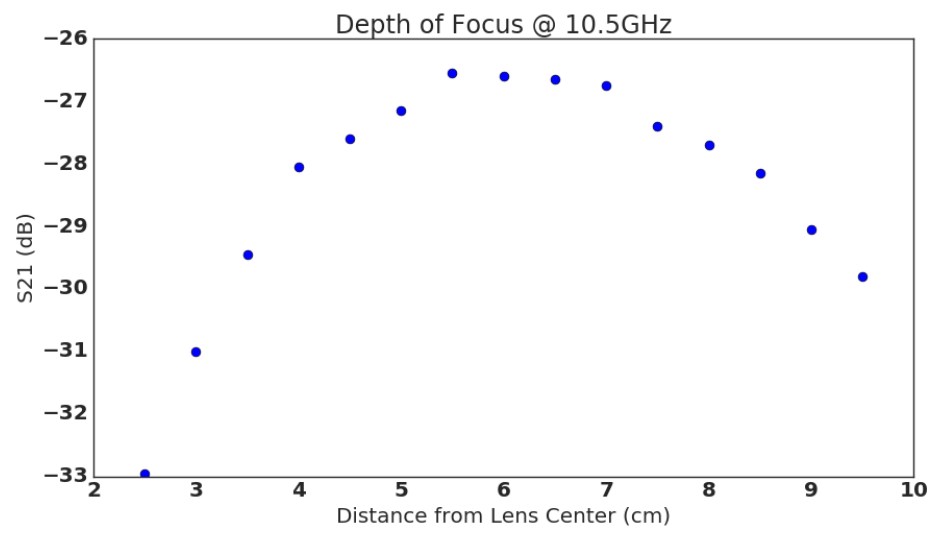

Figure 5.7: Lens Focal Lenght: The focal length of the lens is frequency dependent. Here we collected data near the standard operating point of $10.5 \mathrm{GHz}$. Depth position is given in centimeters away from the center of the lens.

process were raised and the authors noted that the time and rate of deposition had to be limited. An alternative electroplating method prototyped by C. Halseth [7.2] and subsequently improved upon by the contributions in this work will now be presented. This new method is not in risk of causing thermal damage and can be performed at atmosphere in relatively dirty environments without loss of performance. In this work, we replicated their lens using a much lower cost FormLabs 1+ printer. Due to the limited build volume of the printer, each lens layer is fabricted in quadrants and soldered together after plating.

\section{Electroplating Process}

The general copper electroplating process begins with three components; the source, the transport solution, and the object to be coated. An electrochemical potential is applied between the source (anode) and object (cathode) to be coated. Ionic current, facilitated by the transport solution, forces copper ions towards the object to be coated along the path created by the strongest field 
lines from the positive to negative terminals. This implies that the object to be coated is always connected to the cathode and the anode is always connected to the copper source. Copper from the anode is ripped from the source, flows through the copper sulfate and gets redeposited on the surface of the object. This process is described chemically using the half-reactions:

$$
C u \rightarrow C u^{2+}+2 e^{-}
$$

and

$$
2 e^{-}+C u^{2+} \rightarrow C u
$$

The first process describes the reaction between the anode and the copper sulfate transport solution when sufficient energy has been applied to excite the copper atoms into an excited state. Two ions are free to move in the transport solution along with 1 heavy cation. In the reverse process, the +2 cation and two electrons recombine in their ground state and reconstitute a single copper atom on the surface of the object being coated. The ionic transport solution is an aqueous $\mathrm{Cu}^{2+}$ and aqueous $\mathrm{So}_{4}^{2-}$.

\section{Electroplating Results}

This is as far as we need to cover about the electroplating process in this thesis since our main interest in this section is to demonstrate manufacturability of the RF components at atmosphere without the need for a sputtering process and to show that the parts perform as intended. Figure 5.8 shows two variants in the preparation steps for surface metalization of the two objects. Method 1 was used to add metalization to the lens while Method 2 was used to coat the 


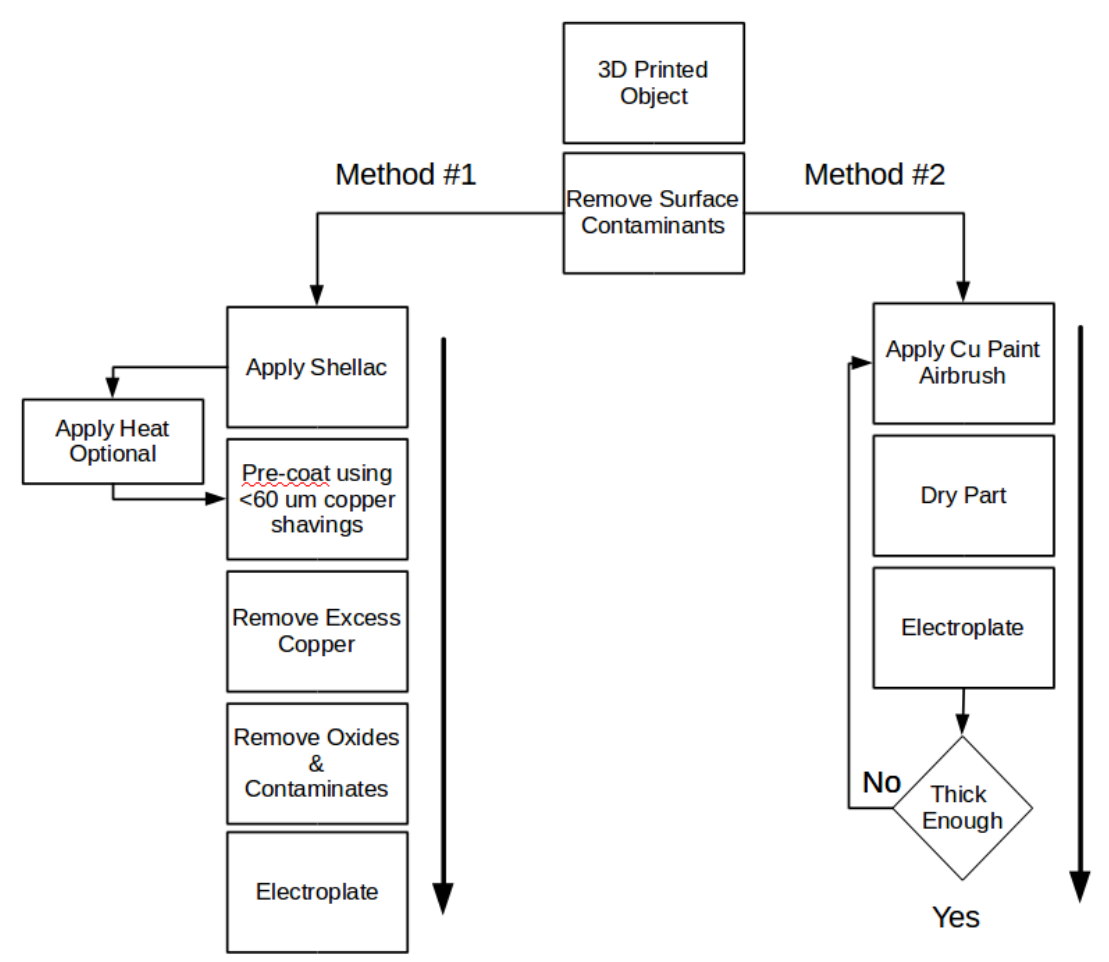

Figure 5.8: Electroplating Process: This flowchart describes two electroplating processes used in the construction of the microwave lens and horn antenna. Both methods are found to be completely successful although Method \#2 has a distinct speed advantage when the surface area of the object being coated is large and shallow. 
interior of the horn antenna. A detailed description of the processes described in Figure 5.8 is provided in Appendix B.

Both techniques were found to produce similar end results after the electroplating process is complete. However, the second method is found to produce more uniform pre-conductive coating layers which is important for uniform electroplating results. Non-uniform pre-conductive coating layers creates potential gradients between the connection point of the cathode and the rest of the object. Since deposition preferentially coats the exposed surfaces with the strongest electric field, there is room for non-uniformity in the final electroplating thickness. We do not consider this to be an issue for two reasons. First, a few microns of coating across the entire surface is already a sufficient coating thickness due to the skin effect at microwave frequencies.

$$
\delta=\sqrt{\frac{\rho}{\pi f \mu_{r} \mu_{0}}}
$$

where we assume no ferromagnetic materials have contaminated the copper so that $\mu_{r}=1$. We will also assume a copper resistivity value of $1.7 \mu \Omega \cdot \mathrm{cm}$ yielding a final skin depth of approximately $0.64 \mu \mathrm{m}$. Once several skin depths have been achieved, the plating uniformity does not significantly change the behavior of the horn or lens since the smallest linear dimension is nearly 3 orders of magnitude greater than the thickness of the coating. Therefore, we place more emphasis on the distribution of coating rather than the actual thickness uniformity. 


\section{Impedance Measurements Summary}

\begin{tabular}{lllc}
\hline Location on Sample & Method & Value & Units \\
\hline & & & \\
Copper Shavings Prior to Electroplate & Method 1 & $1-2$ & $\mathrm{M} \Omega$ \\
Surface Impedance Electroplate & Method 1 & $<1$ & $\mathrm{~m} \Omega / \mathrm{sq}$ \\
Surface Impedance Copper Paint & Method 2 & 60 & $\mathrm{~m} \Omega / \mathrm{sq}$ \\
Internal Neck Impedance after plating & Method 2 & $2-3$ & $\mathrm{~m} \Omega / \mathrm{sq}$ \\
External Impedance after plating & Method 2 & $<1$ & $\mathrm{~m} \Omega / \mathrm{sq}$ \\
\hline
\end{tabular}

Table 5.4: Summary of resistivity measurements results for copper coated antenna components using a 4 point probe.
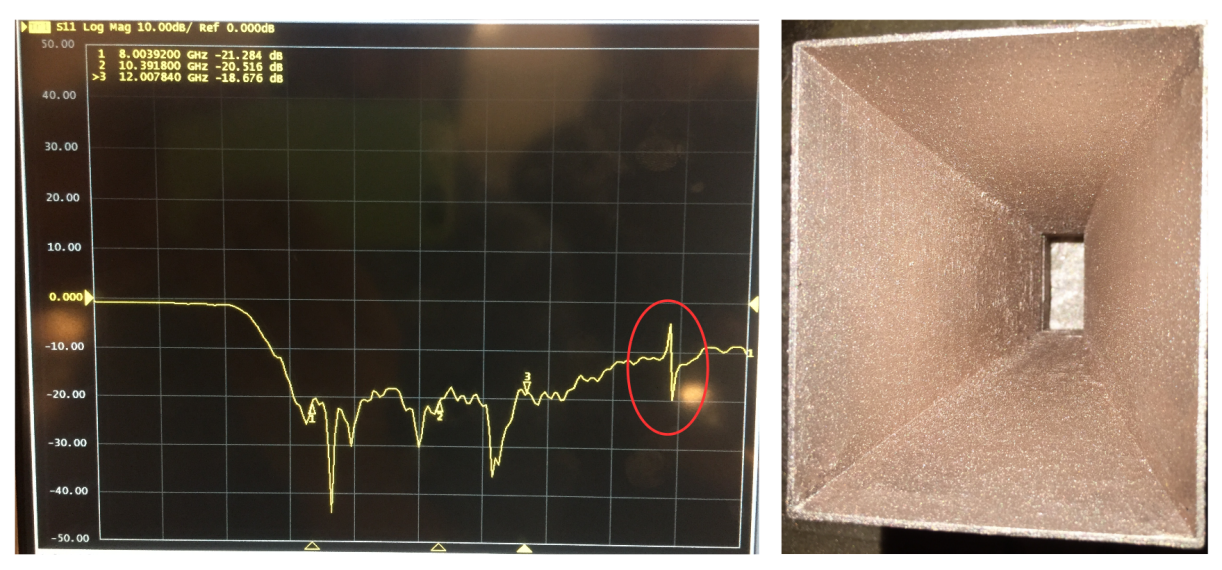

Figure 5.9: Custom Horn Characterization: The optimal recorded coating and performance measurement was observed when using only copper conductive spray without electroplating process. $S_{11}$ is less than $-18 \mathrm{~dB}$ across the entire X-band range. Circled in red is a consistently recurring defect visible in all $3 \mathrm{D}$ printed objects. In this case, the defect is out of band and can be safely ignored. 

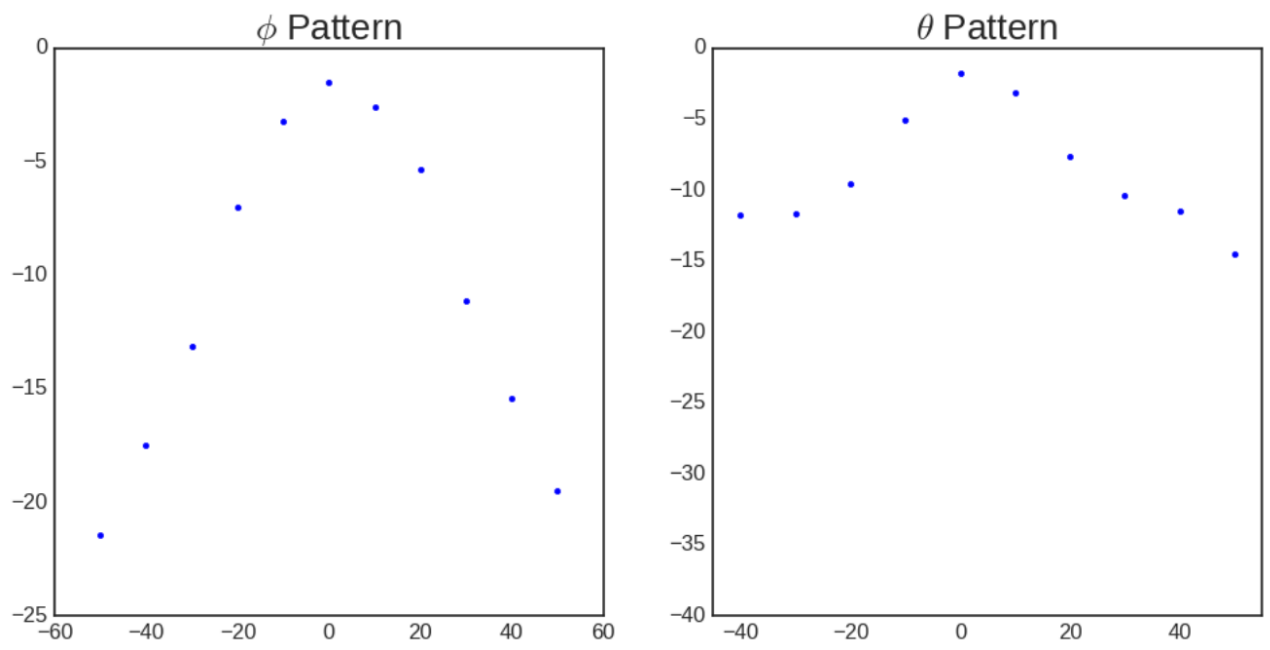

Figure 5.10: Custom Horn Antenna Pattern: Measured antenna pattern with amplitude in $\mathrm{dB}$ and $\mathrm{x}$-variable in degrees.

\subsubsection{Microwave Cavity Configuration}

\section{Microwave Lens Configuration}

Two microwave probe configurations are applied in this thesis. The first configuration exercises the microwave lens shown in Figure 5.6 to focus RF energy onto a small $1 \times 1.5 \mathrm{~cm}^{2}$ spot on the surface of the sample. The planar surface of the lens is directed towards a horn antenna and rotated so that the $\mathbf{E}$ plane of the horn is parallel to the $\mathrm{E}$ plane of the lens. On the concave side of the lens a sample is placed in the focal plane of lens coinciding with a maximum E field probe. The sample should be oriented so that the lightly doped bulk region of the solar cell faces the microwave lens while the 'sunny' side of the cell faces the optical probe. Now both the microwave probe and optical probes have unobstructed views of the sample.

One very interesting design feature added by the use of a microwave lens is that we have now exposed a new way of focusing the $\mathbf{E}$ field probe in the 
sample plane by exploiting the lens focal length frequency dependence ${ }^{2}$. Consequently, we can now write an optimization routine to adjust the microwave signal generator frequency until the focal point of the lens lies in the plane of the sample. In many ways this is preferable to adjusting the sample and lens separation distance mechanically.

\section{Dielecric Probe Configuration}

In the second probe configuration, we remove the microwave lens from the system completely and add a small cylindrical resonant cavity loaded by with a dielectric filler with a relative permittivity $\left(\epsilon_{r}\right)$ between $4.0-10.4$. The purpose for adding this resonant cavity is two fold. First, a high $\mathrm{Q}$ resonant cavity tuned to the $\mathrm{x}$-band frequency filters all signals outside of the passband which may be transmitted through the sample from the external world. Now, we drill a small $5 \mathrm{~mm}$ hole at the center of the cavity normal to the direction of plane wave propagation and load the resonator with a lossy dielectric. We normally wouldn't be able to propagate a plane wave through the reduced aperture since the change in impedance from a cavity of resonant length to a secondary cavity of less than $<\lambda / 4$ results in high magnitude of reflection $\Gamma \sim-1$.

From a waveguide perspective, the fundamental wavelength mode of the loaded cavity is directly proportional to the square root of the relative dielectric constant of the material in the cavity. By introducing a material with $\epsilon_{r}>1$, we are able to effectively 'squeeze' a longer wavelength probe into a smaller cavity. Calculation of the cutoff wavelengths for a cylindrical cavity are in order:

\footnotetext{
${ }^{2}$ This idea has been considered in some detail, but has not yet been fully implemented. There are a number of challenges that must addressed prior to this implementation to ensure that the system has a closed loop feedback.
} 


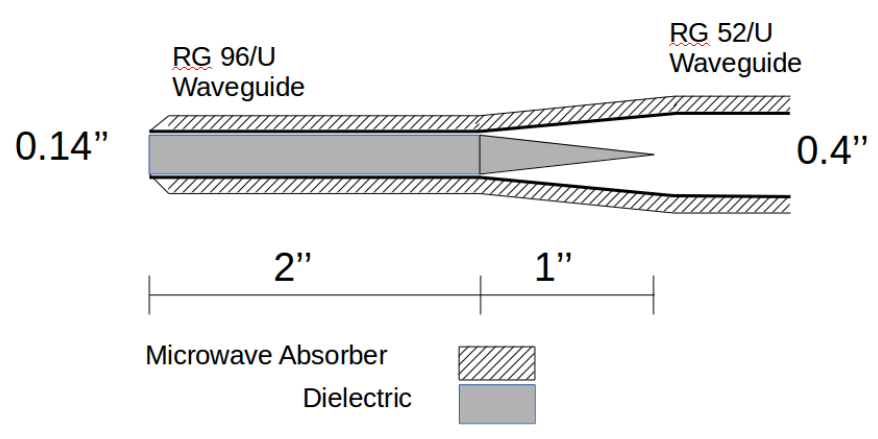

Figure 5.11: Cross section of a waveguide probe examined by Richmond and Tice to improve near-field measurement accuracy [3]. Increasing the dielectric constant increases the cutoff wavelength of the fundamental mode by the square root of the relative permittivity. In the waveguide configuration shown here, mycalex $\left(\epsilon_{r}=11.5\right)$ is used as the dielectric filler.

$$
f_{c}=\frac{p_{11} c}{2 \pi a \sqrt{\epsilon_{r}}}
$$

where $p_{11}=1.841$ for $T E_{11}, p_{11}=2.405$ for $T M_{01}$ and the dependence on $\epsilon$ is shown. If we assume an alumina filler with $\epsilon_{r}=10.4$ and a radius of $2.6 \mathrm{~mm}$, the cutoff wavelength is $2.8 \mathrm{~cm}$ for $T E_{11}$ and $2.1 \mathrm{~cm}$ for $T M_{01}$. The result of this calculation is that we may use a dielectric filler to couple energy from a x-band resonant waveguide to the sample through a narrow waveguide aperture many times smaller than the fundamental mode cutoff wavelength.

With this dielectric probe positioned directly beneath the lightly doped bulk region of the sample, we may now probe the sample in a localized space that is significantly smaller than the dimensions of a waveguide. Furthermore, because we have narrowed the effective sensor aperture, we may scan the microwave beam much more closely towards the edge of the sample. 

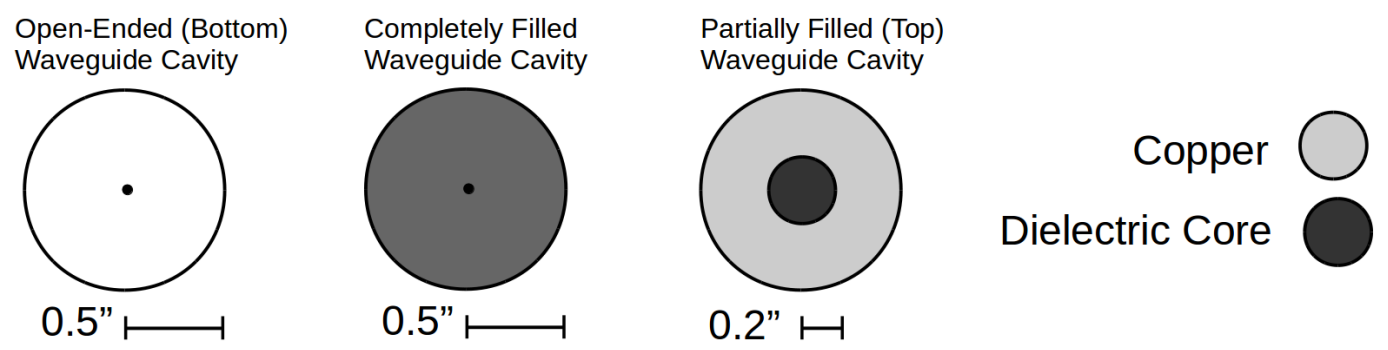

Figure 5.12: Shaped waveguide dielectric antennas were not considered at the onset of this project. Here we settle for a cylindrical waveguide cavity.

\section{RF Shielding}

To further eliminate the disturbances from the external world, we shield the microwave antenna and lens (or dielectric probe) in an RF shielded chamber which provides $35 \mathrm{~dB}$ of attenuation per bounce at $10 \mathrm{GHz}$. The main consideration for doing this is to ensure that the fundamental isolation assumption is approximately true when applying the antenna reciprocity theorem. This amounts to simply verifying that we eliminate as much noise from the external world as possible so that whatever currents and voltages are available in the system are only due to coupling effects between the two antennas. 


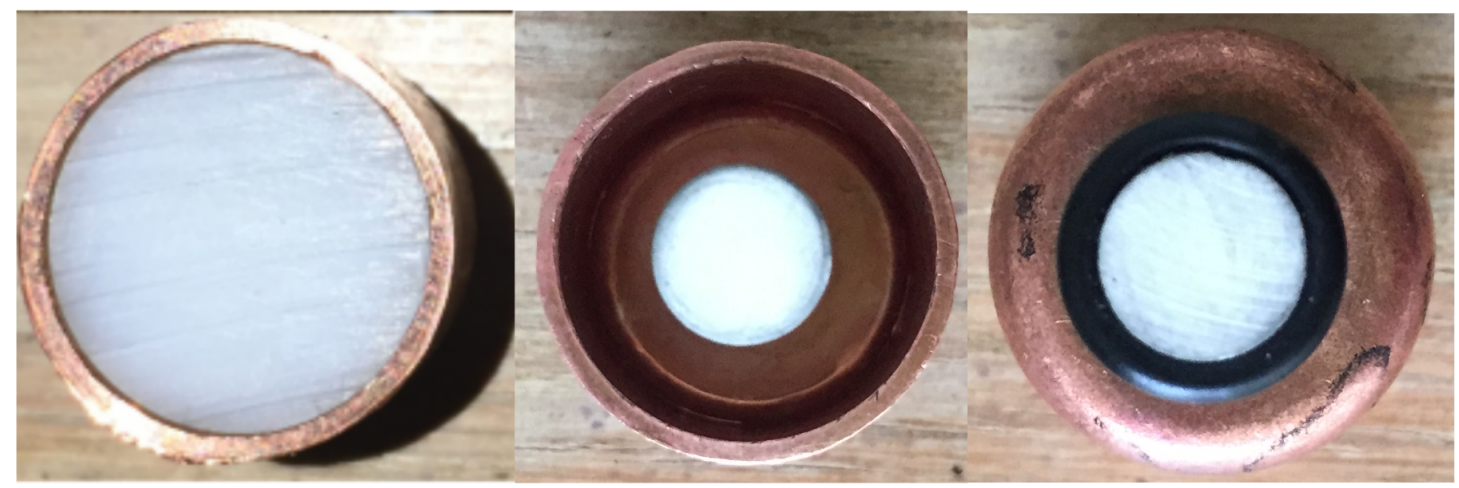

Figure 5.13: Left: A cylindrical waveguide cavity completely filled by a polystyrene dielectric $\left(\epsilon_{r}=4.2\right)$. Middle: A partially filled cylindrical waveguide cavity with the normal vector facing the microwave antenna. Alumina $\left(\epsilon_{r}=10.4\right)$ is used as the dielectric. Right: The top surface of the partially filled waveguide cavity facing the bottom of the solar cell. $5 \mathrm{~mm}, 7 \mathrm{~mm}$ and $13 \mathrm{~mm}$ dielectric cores are confirmed to provide sufficient coupling for either a QE or lifetime measurement.

\subsection{Software Control System}

Python $3 x$ serves as the base language for software development throughout this thesis due to its portability, readability, ease of use, and implementation of an extensive embedded support system for every module. The Python language is intended to allow programmers spanning multiple disciplines to pass code adhering to a strict formatting standard and design philosophy between each other without difficulty. Facilitating the portability condition requires that the language perform all type casting and memory operations under the hood to operate across operating platforms. The code developed in this thesis has been tested ${ }^{3}$ and successfully deployed on the Windows, OS X, and Linux platforms.

Although massive amounts of three dimensional data are generated by the

\footnotetext{
${ }^{3}$ Robust testing from a software perspective was not performed. The use of the word testing only implies that I have configured bidirectional communication and successfully performed measurements using any operating system.
} 
QE routine, the measurement period for each mapped location is on the order of 1 second and the overall bandwidth is low. Lifetime measurements also record massive amounts of time resolved data at $2 \mathrm{MB}$ per measurement site with a medium to high resolution 1000 point map generating over 2GB of data. The data is therefore processed on the fly and binned accordingly at each measurement location. While measurement speed is not a concern in this thesis, measurement integrity and system robustness is a major concern since the interruption of a high resolution map could represent several hours of data collection. Data protection and recovery features are written into the code in the event that a measurement is terminated abruptly or communication between the light engine or data acquisition system gets disconnected. If this event should occurs, the system logs all measurement data acquired during the measurement and automatically resets the connection with the USB interfaces. Re-homing the motion control system is not required (unless the event occurred due to a loss of power), and it is possible to continue acquiring data at the last point of an interrupted recipe.

A robust software control system implementing the modular state machine design pattern was used to design the primary system functionality using the Python Transitions 0.4.3 package ${ }^{4}$. A state machine pattern ensures that the motion control, light engine, data acquisition, signal processing, calibration and display modules can all be tested independently and easily integrated to form a final start-to-finish measurement routine. Note: A module bug was discovered while acquiring very large datasets causing the state machine to suppress data above a certain threshold without raising a fatal error flag. This is due to

\footnotetext{
${ }^{4}$ Author: Tal Yarkoni, Last Commit Date: 2016-12-10, PyPi Repository: https://pypi . python.org/pypi/transitions
} 
the specific module implementation using recursive function calls rather than loop iterators. As such, a custom routine was written for the acquisition very large datasets requiring greater than a 1000 measurement points and a full set of LED channels.

\subsubsection{Measurement Flow Chart}

This subprocess flow chart in Figure 5.14 illustrates how the quantum efficiency and lifetime measurements are acquired in this thesis. Quantum efficiency measurements blink each LED wavelegnth in sequential order until the desired spectrum and number of repeats is acquired before moving to the next position on the sample. A quantum efficiency map is complete once the data for

all wavelengths, measurement repeats and measurement positions have been collected. The QE value is saved by default. Optional storage of raw data is also possible.

The time-resolved lifetime measurement is treated as a special case of the quantum efficiency measurement. For lifetime measurements, a time-resolved curve of a single wavelength probe is recorded with a sample rate between 250-1000 ksps. Since the sheer amount of data that can be acquired in a single map, it is not reasonable to store all data as each measurement could represent GBs of raw data. To reduce the amount of data stored at each measurement site, only the average decay curve is recorded, pulse amplitude and decay time are recorded. 

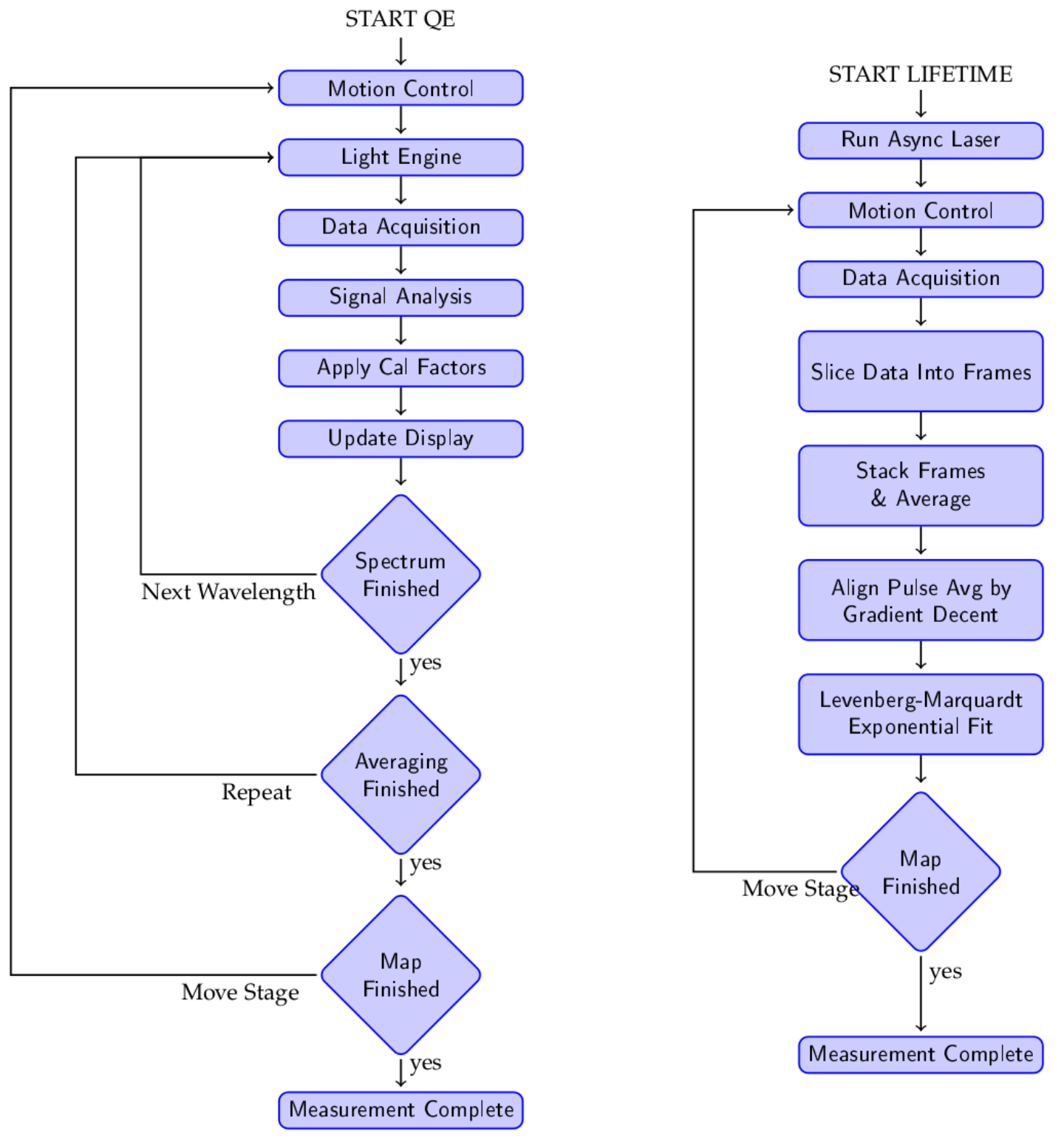

Figure 5.14: Measurment Subprocesses: QE and lifetime measurement processes are described in detail. After the map is complete the results are stored as pickled python objects for later review. In the case of the lifetime measurement, only the frame average, pulse amplitude and decay time subsets are saved. Map recipes are generated prior to the start of a measurement using the custom MapGenerator.py tool. In the event of an unexpected termination, all data currently stored in memory is copied to disk. 


\subsubsection{Software Configuration}

Measurements are configured from a single standalone configuration file with a structure similar to that of a Microsoft Windows INI file. The Python3 "configparser" library is used to read in the INI data structure. However, we have modified the backend so that the INI the configuration parser will understand explicit Python language or raw text formats. Figure 5.15 shows a high level view of the major user configurable settings and how they interact with the different software modules. The full user configuration file is included in Appendix C.

\subsubsection{System Calibration}

The system is calibrated using a single point measurement on a 'known' piece of semiconductor material. As we simply do not have the capability to perform a true calibration, the 'known' piece of silicon is assumed to be of the form shown in the left hand image of Figure 5.17. The fact that we have assumed a calibration curve is not a terrible concern for this work since it is the relative change in quantum efficiency and not the true value that is of general interest for in-line device manufacturers. Linear interpolation is performed on the calibration curve to fit data points lying between any two neighboring values. For each data point, a scale factor is calculated that will bring the measured data point to the ideal value given by the calibration table. Background subtraction is not implemented. 


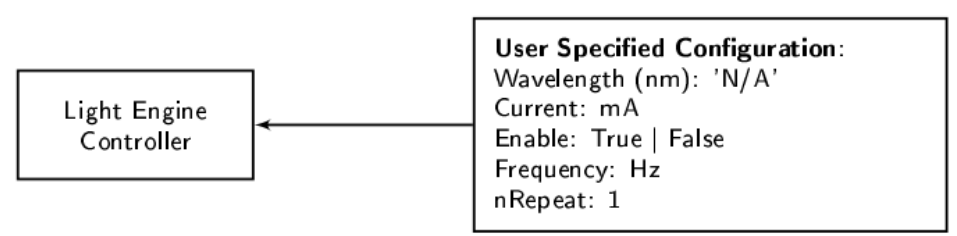

\begin{tabular}{|c|l|}
\hline \multirow{2}{*}{\begin{tabular}{c|} 
Data Acquisition \\
System
\end{tabular}} & $\begin{array}{l}\text { User Specified Configuration: } \\
\text { nChannels: } 2\end{array}$ \\
\cline { 3 - 3 } & Sample Rate: $500 \mathrm{kHz}$ \\
& Digital LPF fc: $1005 \mathrm{~Hz}$ \\
Differential Input: True
\end{tabular}
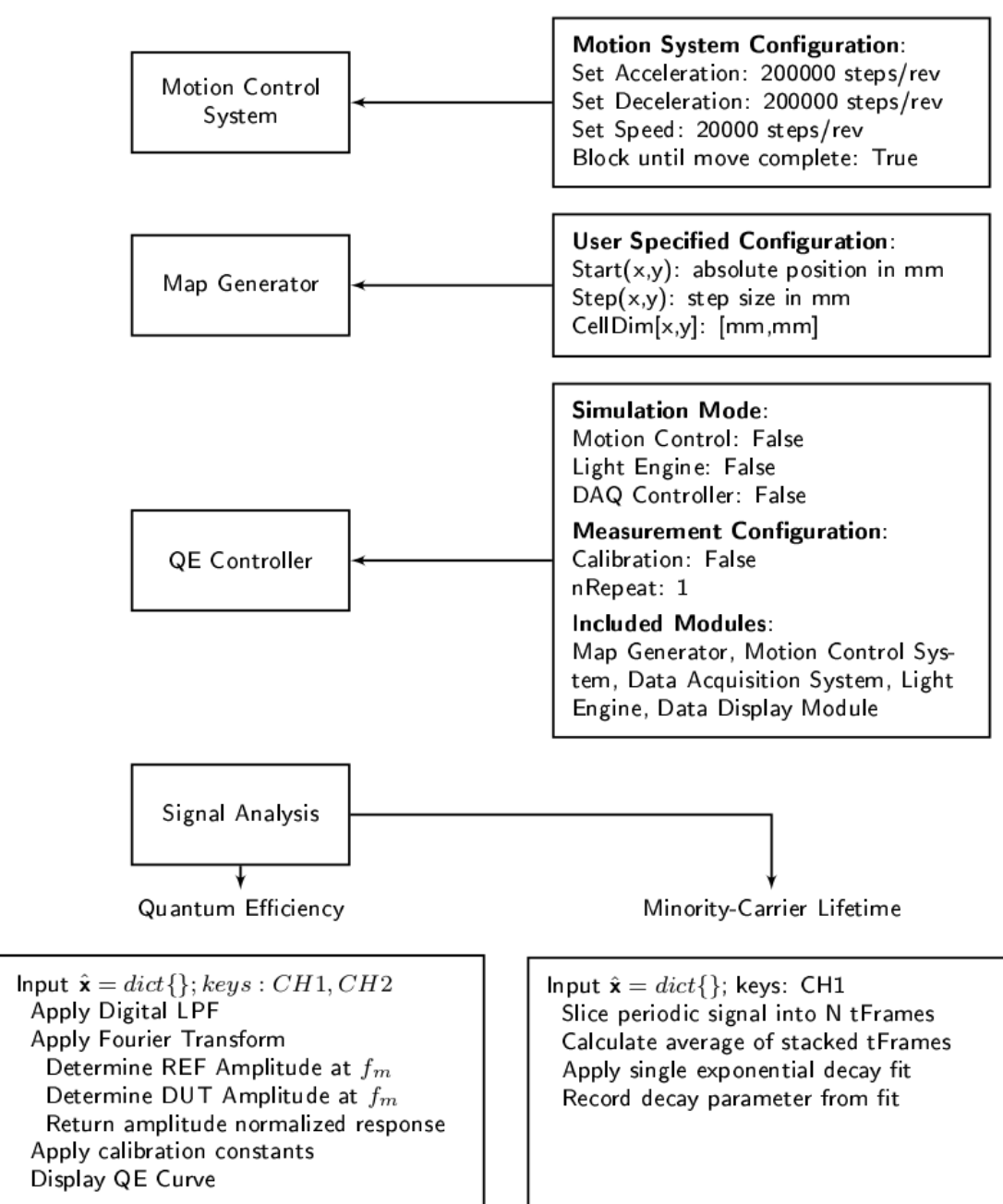

Figure 5.15: Software Components: The major measurement configuration software blocks are shown. All software components can be run and tested independently. Each module is accessed through a well defined class interface. 
5 consecutive measurements shows $3-5 \%$ variability. $T \sim 3 \min$
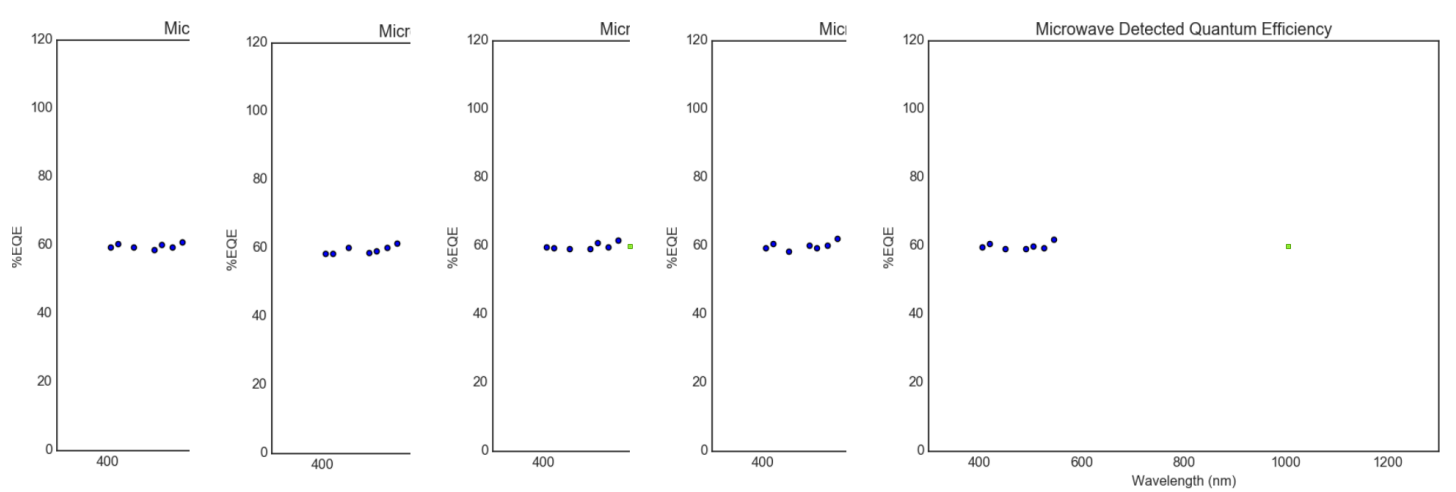

Figure 5.16: First Pass QE Measurement: This figure shows the initial test involving the full integration of both software \& hardware components. A UV bank of LEDs is loaded into the light engine and with an appropriate software configuration file indicating the wavelength, nominal drive current, and pulse repetition frequency. These measurements were taken in rapid succession to demonstrate measurement stability after calibration to a flat line at $60 \%$ EQE.

\subsubsection{First Test Measurement}

In this section, we integrate the software and hardware components in preparation for taking measurement data. A sample is loaded onto the stage platform and LED Bank\#1 containing the 16 shortest measurement wavelengths is prepared. Due to the power non-uniformity issue shown in Figure 5.3, several of the weakest wavelengths are repeated in multiple LED positions to boost output power. The microwave system then feeds into an HP-415E tuned amplifier. The differential output of the amplifier is sampled using a ulta-high input impedance differential analog-to-digital converter. First pass results of the microwave detected quantum efficiency measurement are shown in Figure 5.16 and Figure 5.17.

The thermal stability of the oscillator was not taken into account in the 
first revision of hardware and resulted in measurements that were unrepeatable over a long period of operation. The Analog Devices HMC-C030 oscillator datasheet quotes a thermal drift of $800 \mathrm{kHz} / \mathrm{C}^{\circ}$. A 10 degree shift in temperature results in about an $8 \mathrm{MHz}$ shift in oscillator frequency. Similarly, variations in room temperature from $18 C^{\circ}$ to $21 C^{\circ}$ are sufficient to invalidate the calibration curve.

Several attempts were made to tune and calibrate the system after allowing the oscillator to reach an equilibrium temperature. However, as system tuning can affect power dissipation in the oscillator, the process is necessarily iterative and not very attractive for long term use without thermal stabilization and temperature feedback in the control system. The oscillator frequency is also independently set and not determined by the control system. Furthermore, the output power is fixed and is harmful to the LNA if the system becomes severely unbalanced. For these reasons, the final system implements a digitally controlled source whose phase noise is $-75 \mathrm{dbC} 1 \mathrm{kHz}$ across the entire operating temperature range. The output power is also digitally controlled and can be shut off or attenuated if the system becomes too unbalanced. 

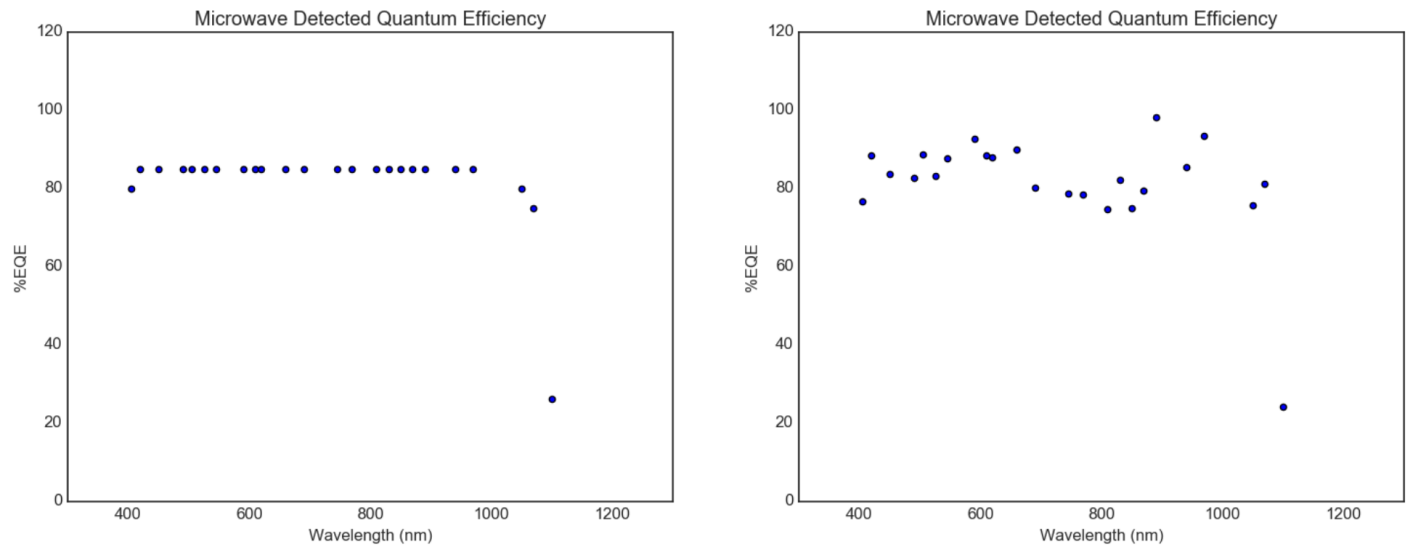

Figure 5.17: Thermal Drift: (Left) The system calibration curve. (Right) A second pass measurement with all four banks of wavelengths loaded into the light engine. Here, we perform continuous measurements of the same sample and position over a 30 minute period. This frame represents a measurement around 25 minutes into the data set and we can notice that thermal drift impacts system stability quite drastically. This issue is now resolved as confirmed by the system repeatability measurements in the following chapter. 


\section{Results \& Analysis}

The results from our non-contact microwave detected quantum efficiency experiments are presented in this chapter with three objectives in mind. First we generate a performance baseline by measuring the signal-to-noise ratio for a single point map over a 250x, $1.2 \mathrm{Hr}$ measurement period for a $10 \mathrm{~mm}$ spot size. Based on the system repeatability, we then estimate the boost in microwave power required to illuminate the entire sample while maintaining the same signal-to-noise ratio. Similarly, we can estimate the increase in signal power required to illuminate the entire sample while maintaining the same signal-tonoise ratio.

Next we focus on quantum efficiency based measurements utilizing the entire $365 \mathrm{~nm}$ to $1100 \mathrm{~nm}$ spectrum and a wide range of optical bias conditions. Linearity of the small signal microwave reflectance signature is demonstrated by varying the optical intensity for a select number of wavelengths using a series of optical filters. Plots of the nominal QE response prior to filtering, QE after optical filters are inserted, and the transmission coefficients of the filters are recorded. Sensitivity to device behavior at different operating points along the characteristic JV curve is also demonstrated. Under strong forward bias, the cell diode begins to conduct and results in a loss of QE signal as expected. At low injection ratios, the sensitivity to photogenerated charge carriers departs from normal contact QE behavior and falls off once a minimum threshold is reached. 
Table of Experiments

\begin{tabular}{lll}
\hline Experiment & Description & Figure \\
\hline \multirow{2}{*}{ Quantum } & a) Single point repeatability test & Table 6.2 \\
Efficiency & b) System linearity test using optical filters & 6.1 \\
& c) Measurement sensitivity to applied bias & 6.2 \\
& d) Minimum bias requirement & 6.3 \\
& e) QE mapping & $6.6,6.4$ \\
\hline Minority-Carrier & a) Amplitude vs. decay mapping & $6.8,6.9$ \\
Decay Maps & b) PL vs. decay map & $6.12, \quad 6.13$, \\
& & $6.14,6.15$ \\
\hline High Resolution & c) JV measurement dependence & 6.11 \\
Mapping & & 6.16 \\
\hline Measurement & a) PL and decay map summary & \\
Summary & & $6.18,6.19$ \\
\hline
\end{tabular}

Table 6.1: Summary of experiments and result figure numbers for non-contact microwave reflectance measurement system.

Finally, evidence that the non-contact measurement is sensitive to many types of defects found in standard silicon solar cells is a strong indicator that the measurement is valid. We demonstrate this using full wafer amplitude and minority-carrier decay maps for a set of six different samples with varying types and severity of defects. In general, the amplitude and decay maps highlight two different sources of defects. However, in some cases, the decay maps can be a more robust form of the measurement as demonstrated on Sample 7. Decay maps of each of these samples are compared with an associated photoluminescence image. There are many cases where the defects recorded by the microwave reflectance measurement do and do not correlate with the photoluminescence image. We believe that this discrepancy can be accounted for, at least in part, by the difference in operating point for each type of measurement. This conclusion is supported by maps illustrating defect masking under specific bias conditions. A tabular summary of experiments performed 
is shown in Table 6.1 .

\subsection{System Repeatability Measurements}

We characterize the quantum efficiency measurement repeatability by measuring the $1 \sigma$ signal-to-noise ratio (SNR) using a stationary sample over the course of a 1.2 hour period. The measurements were made using a $1.5 \mathrm{~cm}^{2}$ spot size with all leds pulsed at the maximum continuous current as indicated by their respective datasheets. Measurement repeatability is strong in the blue region of the spectrum and degrades progressively as we approach the IR portion of the spectrum as shown in Table 6.2.

The observed signal-to-noise ratio is nearly sufficient for most wavelengths in order to meet the in-line application goal described during the introduction. Based on the current microwave signal generator power output and optical power available from the light engine, a 10x performance gain is easily expected using a $+20 \mathrm{dBm}$ signal generator and by filling all remaining LED channels with repeated wavelengths. 


\begin{tabular}{cl}
$\begin{array}{c}\text { Single Point Repeatability Performance (250x) } \\
\text { Wavelength (nm) }\end{array}$ & Measurement SNR \\
\hline 365 & 11 \\
405 & 192 \\
420 & 494 \\
450 & 572 \\
490 & 240 \\
525 & 217 \\
620 & 227 \\
690 & 94 \\
770 & 102 \\
810 & 118 \\
830 & 88 \\
850 & 83 \\
870 & 76 \\
910 & 98 \\
970 & 89 \\
1050 & 82 \\
1070 & 72 \\
\hline
\end{tabular}

Table 6.2: Summary of measurement repeatability for the microwave detected quantum efficiency system as a function of wavelength. The SNR is calculated using $1 \sigma$ noise. 


\subsection{Quantum Efficiency}

In this section, we will focus on two experiments that affirm the validity of our measured results. The first experiment examines the response of the system as a function of the insertion loss due to various optical filters placed in the path of the main column. This experiment is intended to test two possible points of contention. First, is the system response linear; i.e. are we really detecting the small signal change in conductivity due to photogenerated carriers? Second, is the system sensitive to ground-loop coupling or radiative coupling to the modulated LED light engine giving rise to a potential source of measurement error? The second experiment is intended to test the signal sensitivity as a function of the DC operating point of the device. A single blue wavelength slice from the bias dataset is chosen for study. But clearly, any slice of the dataset shows will show the same trend.

\subsubsection{QE Response with Optical Filters}

Five of the measurements behave as expected showing appropriate attenuation according to the transfer characteristic of the optical filter. Unexpectedly, this measurement also demonstrated an error in the LED configuration file as shown by the $520 \mathrm{~nm}$ bandpass filter in Figure 6.1. The observed response at $520 \mathrm{~nm}$ illustrates that LED signal strength was attenuated by $20 \%$ greater than expected. An OceanOptics spectrometer was used to confirm that the peak LED emission was outside of the filter response with a strong tail leaking through the filter.

This is the only measurement where the negative index metamaterial lens was used to collect data. In this case, the sample, microwave lens, horn antenna 

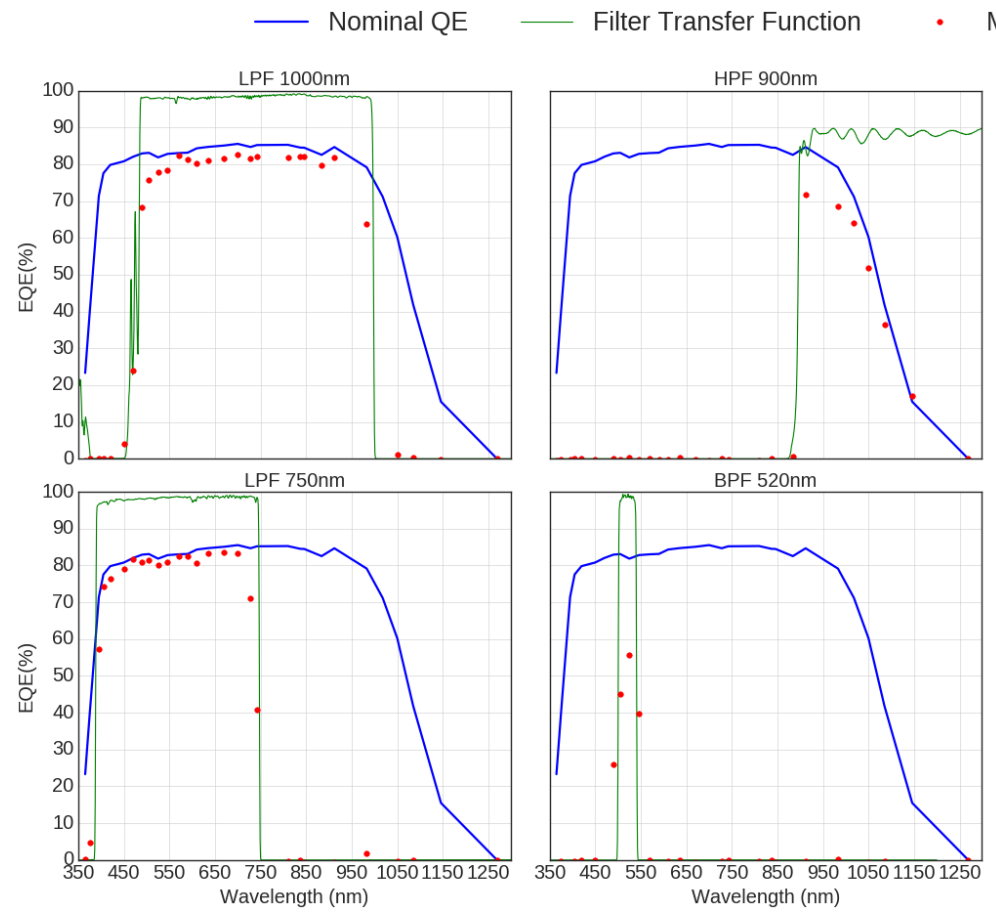

Measured QE w/ Filter

Figure 6.1: Optical Filter Measurement: This measurement verifies that channel crosstalk, ground loop coupling and radiative coupling to the light engine are not observed in the data. The microwave detected signal is only observed as a function of the photogenerated carriers in the sample. The nominal QE response without any filter is shown in blue. The transfer function of each successive filter is plotted in green and the measured response shown in red. This data is acquired using the NIR lens with $1.5 \mathrm{~cm}$ diameter optical probe.

and optics were positioned horizontally on a table with slide rails setting the relative distance between each of the objects. Datasets were acquired using a 30x repeat for each QE curve acquired.

\subsubsection{QE Response with Applied Bias}

Addition evidence supporting the validity of the microwave QE measurement is obtained by varying the DC light bias incident on the sample. Conventional contact QE measurements are performed in the dark and at short-circuit conditions. However, it is not uncommon to vary the bias of a cell to measure QE 
at different points in order to reconstruct a JV curve. Such a test of the QE response to different points along the JV curve is believed to contain information relating to device performance early in the morning or late in the evening.

Results Out of Calibration Relative Measurement Between Bias Points Only
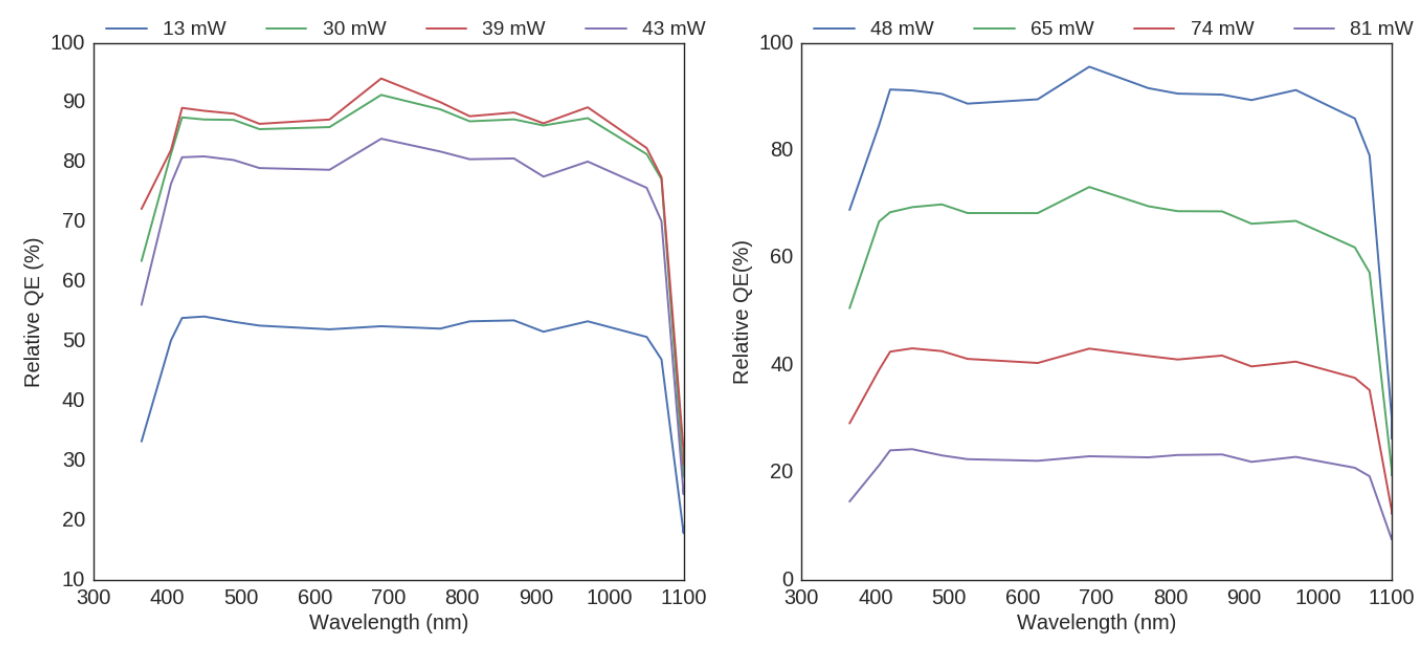

Figure 6.2: Optical Bias Measurement: The effect of a DC forward optical bias in QE measurements is significant. Starting from the lower left QE curve in clockwise fashion, an optical bias of $13 \%, 20 \%, 39 \%, 43 \%, 48 \%, 65 \%, 74 \%$, and $81 \%$ of 1 Sun illumination is applied to the sample. Two effects are observed here; 1) photoflattening in strong forward bias and, 2) low dark measurement sensitivity.

The effects of DC forward optical bias on QE in Figure 6.2 are significant. Quantum efficiency curves are shown for optical power applied in terms of the $100 \mathrm{~mW} / \mathrm{cm}^{2} 1$ Sun standard. Starting from the lower right-hand QE curve at $81 \%$ of a Sun illumination, the small signal quantum efficiency curve is suppressed as the junction is forward biased and beginning to conduct. Moving in a counter-clockwise the effect of reducing bias illumination is demonstrated with the expected rise in small signal amplitude. This trend continues until we turn down the bias illumination to about $40 \%$ of 1 Sun. At this point, the 
microwave detected measurement departs from standard QE measurement behavior and the signal amplitude falls off once again. Figure 6.3 illustrates this effect.

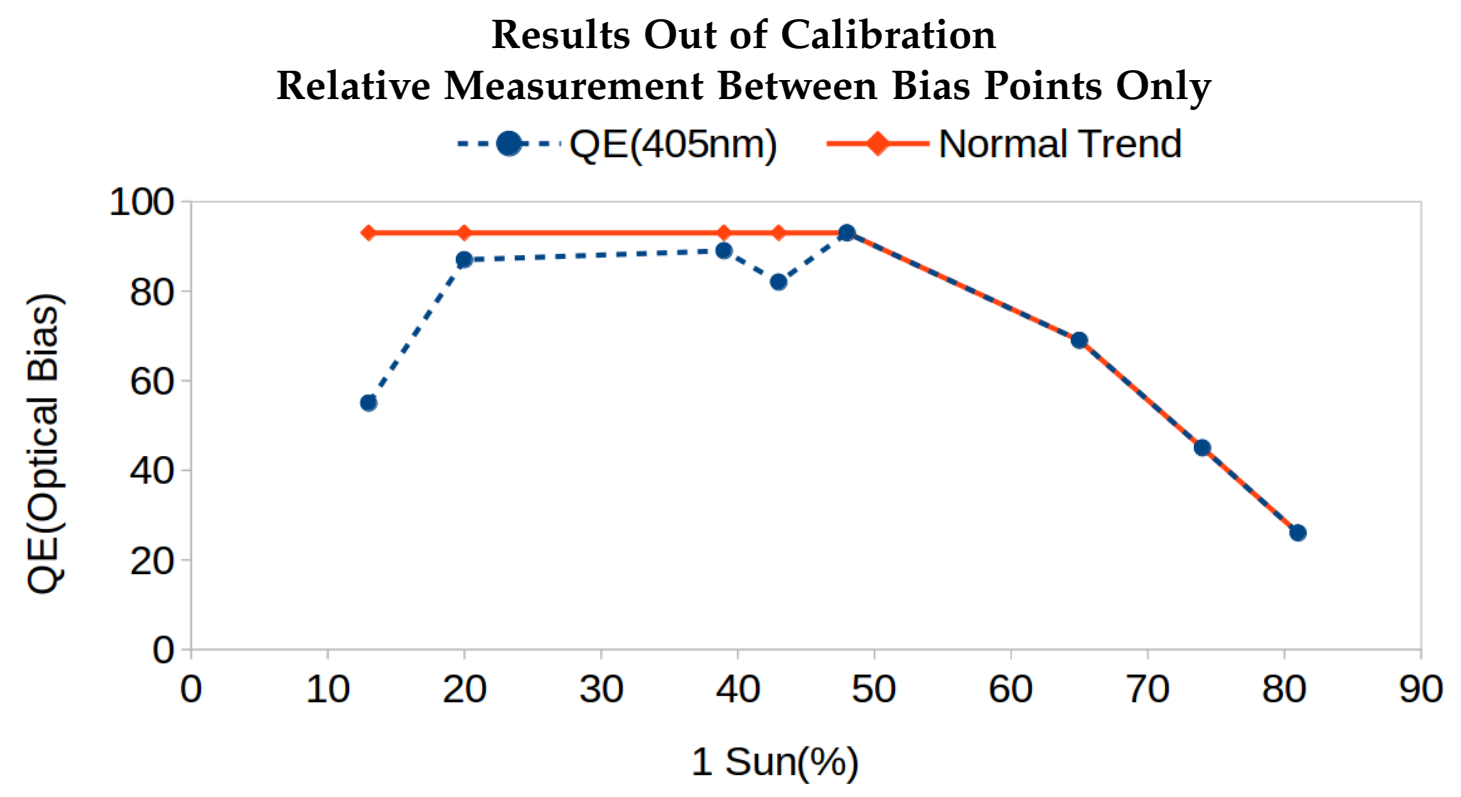

Figure 6.3: 1D Optical Bias Measurement: A slice of the biased quantum efficiency curves along the 405nm axis profiles the QE curve as a function of DC optical bias. The optical bias shifts the local operating point of the cell along the JV characteristic.

\subsubsection{QE Maps}

The previous measurements in this chapter were all taken at a single measurement site. Assembling maps of the quantum efficiency across the surface of the solar cell can be extremely useful in identifying spatial nonuniformity problems in the manufacturing process. Such problems are often exhibited as slow gradients from transitioning from one region of the sample to another. Mapping of a small localized region is also useful for identifying isolated point defects and surface scratches that would otherwise be obscured in a single 
point. Finally, application of Beer's law describing the z-distribution of a photogenerated carrier population in the sample allows depth dependent defect information to be extracted from QE data sliced along the wavelength axis. Blue photons are absorbed very near to the front surface of the sample generating a high carrier population in the first few microns of the sample. Long wavelength red photons have a greater distribution towards the back of the sample. Tracking variations in mapped quantum efficiency for short and long wavelength photons is helpful in identifying a handful of front and back surface defects.

For this study, we choose $405 \mathrm{~nm}, 690 \mathrm{~nm}$, and $1070 \mathrm{~nm}$ to examine the front, bulk and back surfaces of the solar cell. When analyzing these maps, we want to be perfectly clear that the system does not have a true calibration to a known standard. All measurements can only yield relative information between both the wavelength and sample ID axes of the complete dataset. Nevertheless, these types of relative measurements are extremely useful for inline applications where only variations from sample to sample are of primary concern.

\section{Sample 5}

Strong variation in blue response is observed near the edges of the cell along with several patchy areas near the center of the cell. Moving into a deeper slice, the edge effects are less pronounced although defects on the left and right side of the sample has become more pronounced. At the back surface of the sample, we see that the response if fairly uniform at the center of the cell with several major defects correlating with the left and right side defects observed in both 


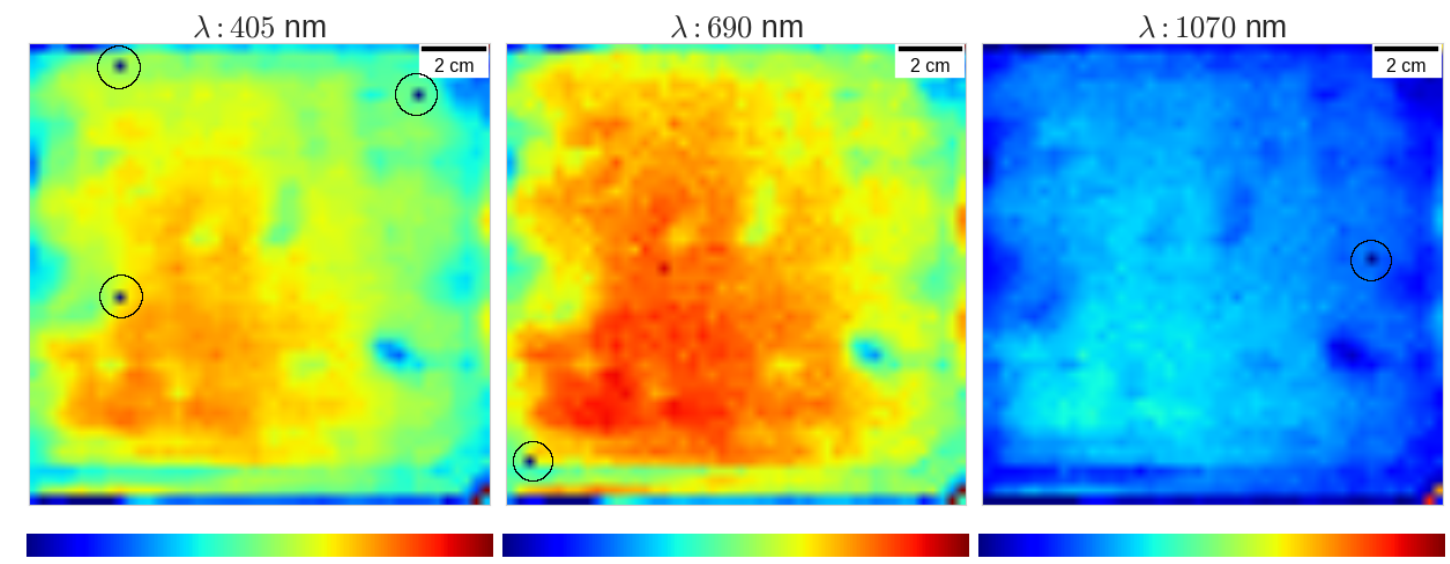

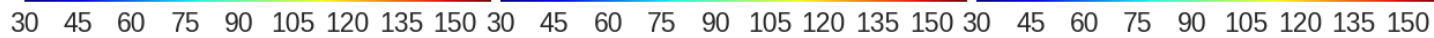

Figure 6.4: Quantum Efficiency Map: Three wavelength (UV-VIS-IR) slices of a QE dataset acquired on Sample 5 show strong spatial between the ultraviolet and visible wavelengths. Five measurement artifacts are observed and marked by black circles. The map resolution is $3 \mathrm{~mm}$ per pixel.

the UV and Vis measurements. The change in edge performance from UV to visible indicates a possible anti-reflective coating problem on the surface of the sample while the observation of strong defects in all map layers on the left and right edges indicate scratching or microcracks that penetrate all layers.

Figure 6.5 inverts the color scale of Sample 5 to highlight specific regions more clearly. In particular boxes around localized edge defects, track marks across the lower $1 / 3$ rd of the sample and a scuff mark in the middle of the sample show three distinct types of defects.

\section{Sample 10}

The most pronounced defect visible in sample 10 occurs along the upper left hand corner of the cell. Strong recombination at the back surface of the sample lowering the device's current contribution throughout the entire boxed ROI is markedly different from the front surface response in which three distinct 


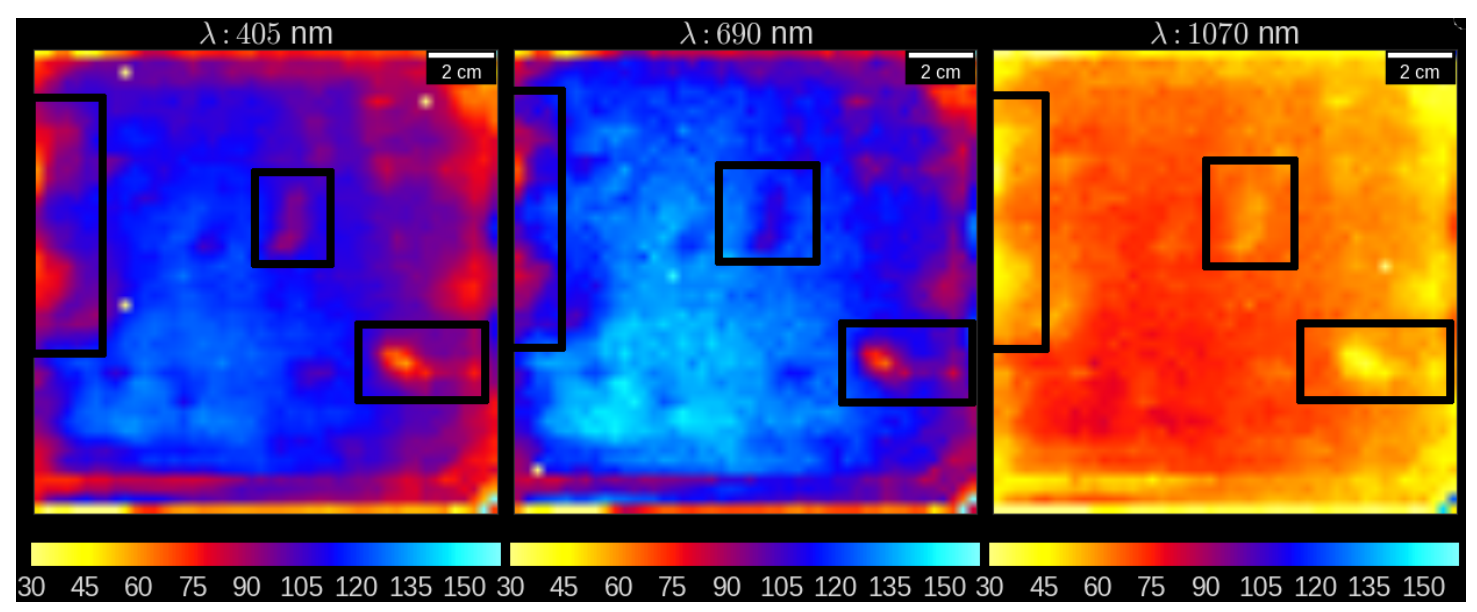

Figure 6.5: Quantum Efficiency Map: Inverting the color scaling of Figure 6.4 (Sample 5) highlights additional cell defects as indicated by the ROI boxes. Defects shown persist in all layers of the measurement indicating a severe problem. Within the ROI of the lower right hand defect, we observe a $20-30 \%$ loss in available carriers across all wavelengths between the defect center and the edge of the ROI.

defective regions are visible within the ROI. When put another way, defective area expands as we penetrate deeper into the sample with a longer wavelength probe. Therefore, the problem likely exists on the back surface of the sample. Visual inspection of the cell by eye does show scratches along the back surface. This ROI is studied in detail later in this chapter using high resolution (1 MP) photoluminescence imaging and single wavelength QE scans at $7 \mathrm{kP}$.

Aside from the defects observed at the corner of the cell, machine handling track marks running the length of the cell about $1 / 3$ rd of the way up from the bottom are also identified. Evidence of track marks is also visible in the PL and high resolution measurements. Note that the sample will be rotated by 90 degrees in future measurements. Since the defects stay with the sample through a rotation, this is further evidence that the defects are real. 

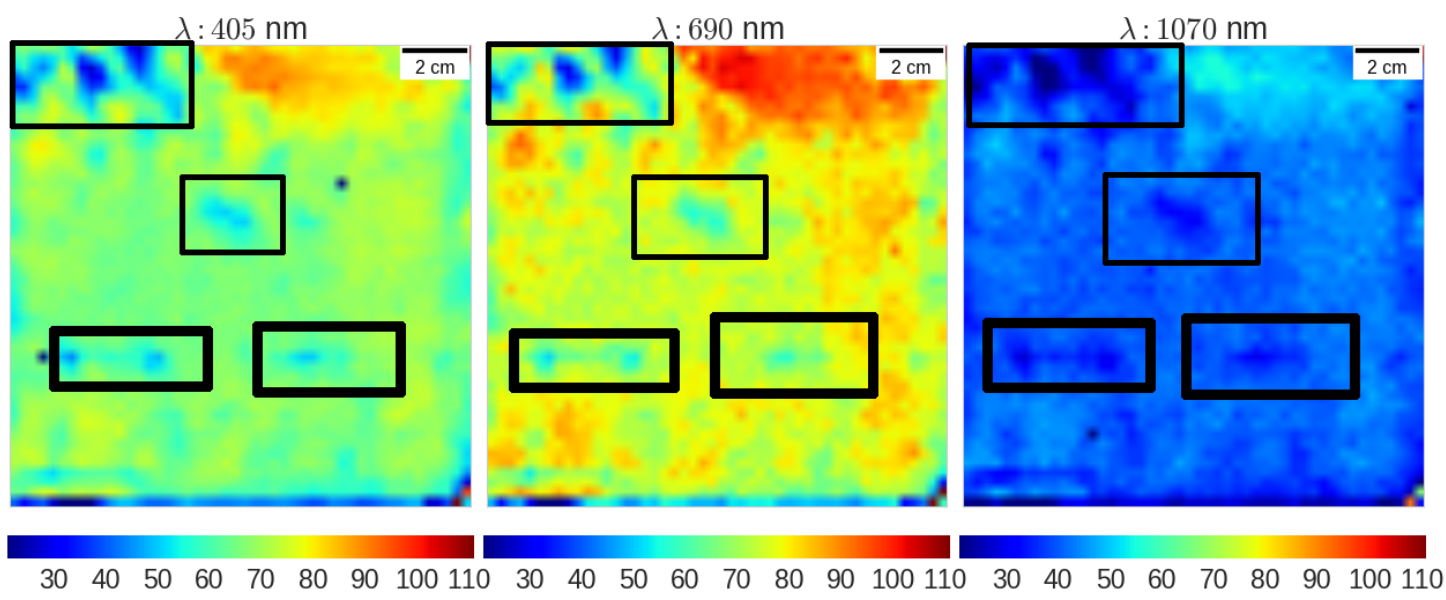

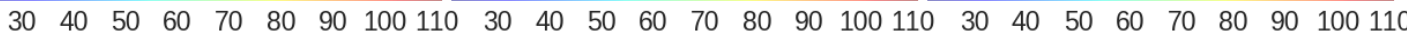

Figure 6.6: Quantum Efficiency Map: Three wavelength (UV-VIS-IR) slices of a QE dataset acquired on Sample 10 with a $3 \mathrm{~mm}$ map resolution. The highlighted boxes indicate wafer handling issues while underperforming regions indicate potential issues with the deposition process. Thick boxes along the lower 1/3rd portion of the sample show skid marks along the direction of travel when the cell is moved from station to station. The lighter boxes indicate scuff marks and scratches working inwards from the edge of the sample. This sample is revisited in PL, decay and high resolution amplitude maps later in this chapter.

\subsection{Microwave Detected Minority-Carrier Decay Maps}

The minority-carrier lifetime is defined by the time that it takes for the excess charge carriers to fall by a factor of $1 / e$. A reasonable silicon solar cell will have a characteristic average minority-carrier lifetime on the order of $100 \mu s$. Really good cells will have longer lifetimes, but certainly should not be expected to live for longer than $1 \mathrm{~ms}$. Assuming that these cells behave no differently, then a $1 \mathrm{kHz}-50 \%$ duty cycle pulse train should be sufficient to allow all of the charge traps to fill ${ }^{1}$ and allow for enough time for the carriers to decay to the dark state before the next pulse arrives. The integration time for each point can

\footnotetext{
${ }^{1}$ Some lifetime measurements operate in the quasi-static regime where the laser is left on for some time to fill defect states. Only once these states are filled is the laser turned off and a lifetime measurement recorded. This behavior is more representative of the actual behavior of the device under full solar illumination rather than the short duty cycle pulsed-mode measurements.
} 
be adjusted as needed for higher signal-to-noise ratio or faster measurement speed.

For the purposes of this method description, we will assume a 1s integration time. Since the signal is a periodic function with sufficient wait and dwell times before and after each decay curve, the entire 1s measurement can be considered as 1000 equivalent lifetime measurements. We use this periodicity to slice the $1 \mathrm{~s}$ measurement into 1000 equal frames where they are then averaged into a single measurement. The purpose of this exercise is to reduce the number of fitting functions that we are required to compute for each lifetime measurement. The end lifetime measurement applied in this fashion is significantly more robust than applying the fitting function to a noisy dataset. Figure 6.7 is a visualization demonstrating the method with real data.

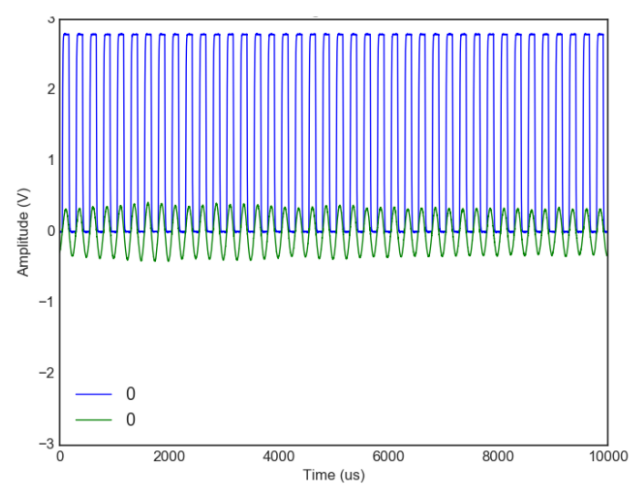

(a) Signal after narrowband filter resonant filter. Filter bandwidth is $15 \mathrm{~Hz}$ with a $1 \mathrm{kHz}$ tone. Narrowband detection used for QE measurement.

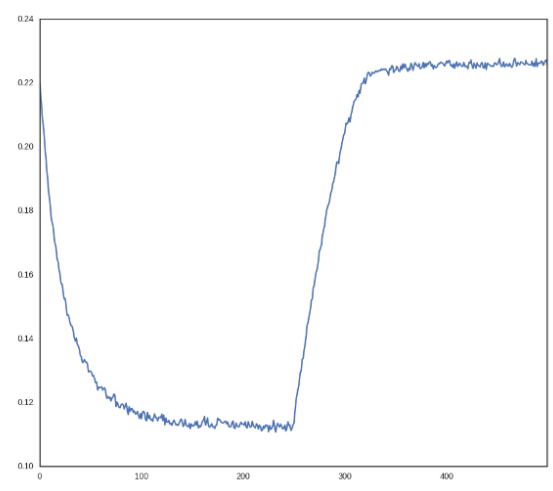

(b) Lifetime measurements are recorded wideband with a time domain resolution of $2 \mu \mathrm{s}$. Average $\tau$ for our sample set is on the order of $45-85 \mu s$.

Figure 6.7: Wideband detection of lifetime decay curve. Since the signal is known to be periodic, we break apart the $\mathrm{N}$ pulse frames in (a) and stack them up into a single pulsed average shown in (b). The Levenberg-Marquardt exponential fit algorithm is then applied to extract the decay time. 


\subsection{Amplitude vs Decay Maps}

This section shows that the decay map is more robust at identifying defective regions on the sample and exhibits less granularity from pixel to pixel than the corresponding amplitude map generated from the same dataset when the bandwidth is large. We expect the amplitude map to exhibit a higher noise characteristic since small shifts in receiver sensitivity and microwave carrier rejection can be exhibited as anplitude noise. Decay maps measuring the 1 /e decay time relative to some starting value are not affected by the previously mentioned amplitude affects. Therefore we can achieve higher signal fidelity and defect sensitivity using decay maps when the bandwidth is large. Cell artifacts are more easily distinguished from microwave induced artifacts since the decay time is purely solar cell device characteristic if the measurement bandwith is sufficient.

However, if time-resolved measurements are not required, then the noise can be reduced by the square root of the measurement bandwidth. In our case, we can improve the SNR by 550x by moving from a $300 \mathrm{MHz}$ bandwidth to a $1 \mathrm{kHz}$ bandwidth. Thus, whenever possible the measurement bandwidth should be limited to the minimum bandwidth required by the measurement and no further. The following two measurements illustrate the advantage of using decay maps at wide bandwidth.

\subsubsection{Sample 2 Characterization}

The two most prominent defects apear along the left hand side of Sample 2 in Figure 6.8. The most severe defect occurs at the edge of the sample in both the amplitude and decay maps. The second most prominent defect visible to 
both the amplitude and decay maps occurs in the middle of the lower left hand quandrant of the solar cell. These defects are well correlated both in relative severity and spatially.

Several defects also visible in the decay map that are not visible in the amplitude map. This defect is observed as a streak on the left hand side of the cell running between the middle $2 / 3$ rds of the cell. We can also resolve two point defects along the right hand side of the sample of the decay map. Both of these defects $1 / 3$ rd and $2 / 3$ rd of the way up from the sample likely indicating the beginning of track mark degradation considering that one mark is perfectly aligned with a similar mark appearing laterally on the other side of the cell.

\subsubsection{Sample 7 Characterization}

Sample 7 shown in Figure 6.9 demonstrates a distinct pattern in both the amplitude and decay maps that is suspected to be due in part, to a real change in sample characteristics in this region. However, the effect is also believed to be exacerbated by a measurement artifact induced by a shift in the carrier rejection circuit away from a null.

The shape of the amplitude defect does give some sense that the artifact is at least partially due to real cell effects. We would expect to see a gradient distribution if the artifact was position or chuck related. We also note several jagged arms protruding from the top and bottom of the defect further adding to the idea that there is some sample relation here. Finally, the artifact is quite discontinuous over the entire boundary, i.e. at not point does the artifact make a smooth transition to the well behaved area of the sample.

Contrary to the amplitude map, the decay map is able to exctract useful 

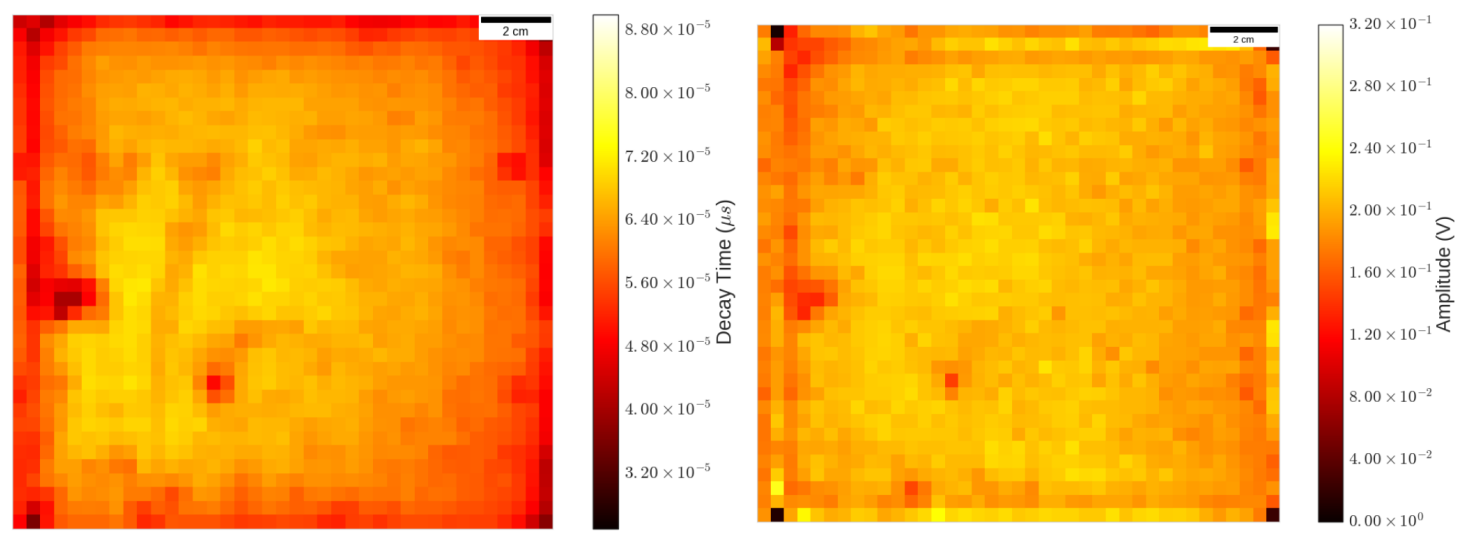

Figure 6.8: Amplitude vs. Decay Map: Comparison of the microwave reflectance signature for the decay (left) and amplitude (right) of Sample 2. Both images are derived from the same dataset showing distinct separation of amplitude and decay term defects.

information from inside of the dead region of the artifact. It is somewhat noisier than outside of the region due to use of a fewer number of adc bits. The measurement is more interesting in that the decay map artifact outlines the affected region only on the top and right-hand boundaries. The left-hand boundary is unaffected. 

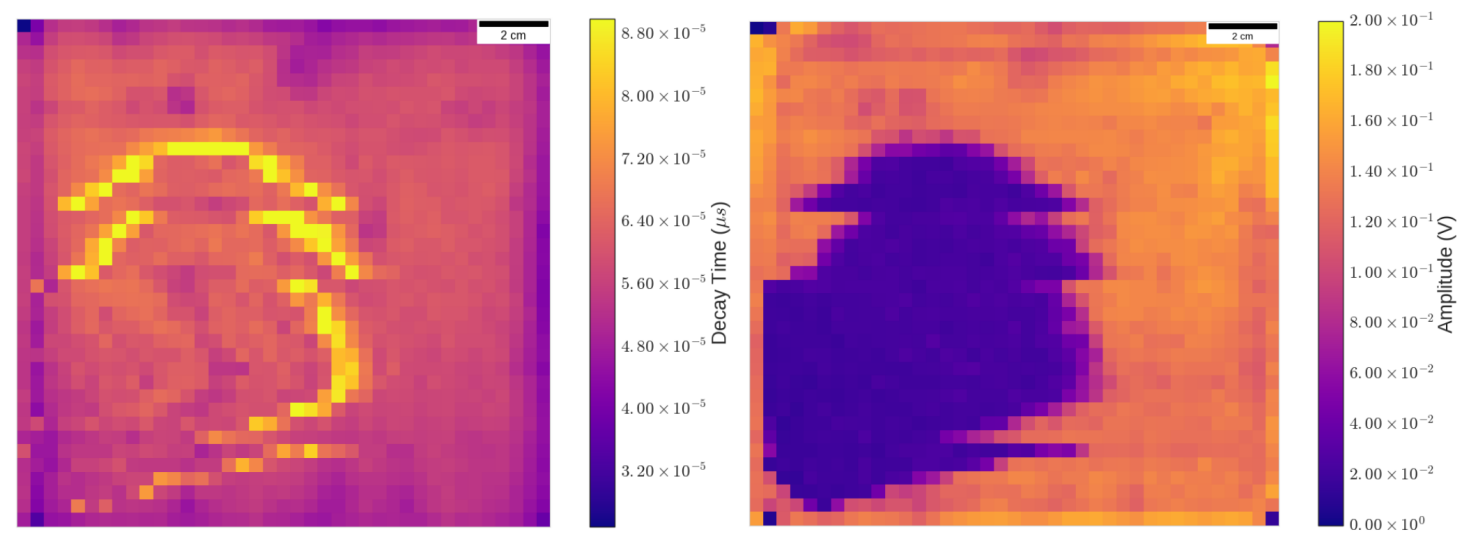

Figure 6.9: Decay and Amplitude Comparison: The microwave reflectance signature for the decay (left) and amplitude (right) of Sample 7 is shown. In this instance, the decay algorithm appears to be more robust than the amplitude map at identifying defects. We note that the decay map is able to extract useful information from inside the dead region of the amplitude map. 


\subsubsection{Low-Injection and High-Injection Considerations}

The previous three samples demonstrate many similarities between the photoluminescence images and their microwave counterparts. At the same time, there are also many notable features that do not correlate well with each other which we think can be explained by studying the operating point of the device in each measurement. Figure 6.10 uses the JV characteristic of the device to illustrate this point for both measurements.

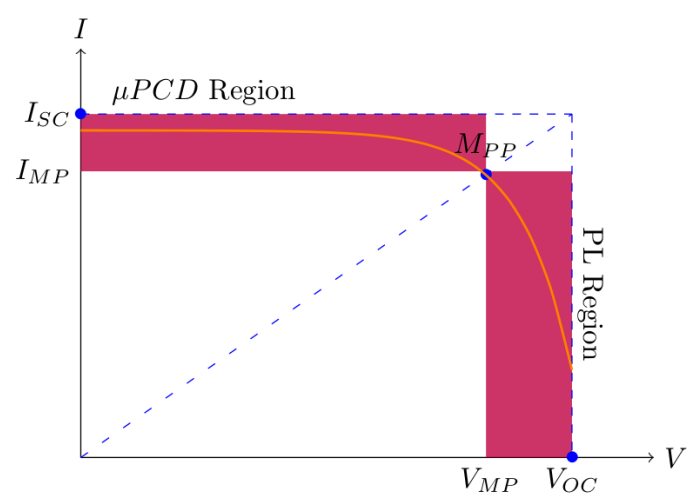

Figure 6.10: The JV curve is useful for showing the operating region for the two different measurements. The photoluminescence measurement occurs under strong forward bias conditions with an illumination intensity often greater than 1 Sun.

We observed the small signal amplitude dependence on bias conditions in Figure 6.3 for a single point using a $405 \mathrm{~nm}$ probe earlier. A more effective demonstration of small signal sensitivity to bias conditions in the microwave reflectance signature is shown in Figure 6.11. Here, we map out an entire solar cell (Sample 4) twice at two different points along the JV curve. On the left, we biased the sample with approximately $30 \mathrm{~mW} / \mathrm{cm}^{2}$. The amplitude map to the right side of the same figure is only biased to $50 \mathrm{~mW} / \mathrm{cm}^{2}$.

There are a few points of interest in these two measurements: (1) The higher bias point measurement has a lower average amplitude as expected as the pn 

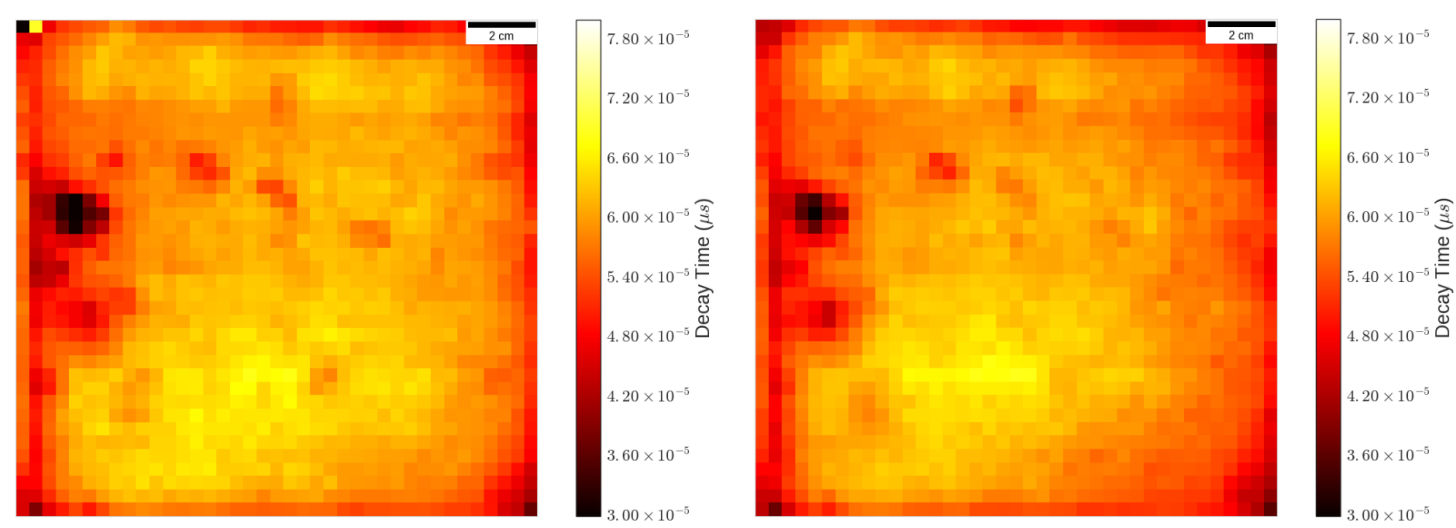

Figure 6.11: Slight optical bias is used to highlight the trapping effect. Slight increase in bias illumination begins to fill trap locations resulting a more uniform device behavior. The sample on the left is shown with an increased broad spectrum white light bias source.

junction begins to conduct current. (2) The relative strength of defects shifts with operating point. Defects may appear in the low-injection regime of operation as surface trapping centers begin to dominate.

\subsection{Photoluminescence Imaging vs Decay Maps}

Photoluminescence (PL) images of the samples are quite effectively locate defective regions of the sample. Any correlation between microwave maps, either decay or amplitude mode, should be taken with a grain of salt due to the difference in operating point discussed in the previous section. Nevertheless, these images are useful for visualizing certain defects and confirming that the observed microwave defects are sample related and not artificially injected by the instrument. Consider the following three PL and microwave detected decay comparisons: 


\section{Sample 1}

A decay map and PL image of Sample 1 are shown in Figure 6.12. Unfortunately Sample 1 is also the noisiest sample of the lot. One principle spot defect is clearly visible on the right side of the sample. Careful inspection of the PL and decay maps show lines running the length of the sample in both images. These vertical lines are not expected to be due to track marks as the position does not line up with the expected track position roughly $1 / 3$ of the way in from both sides of the cell.
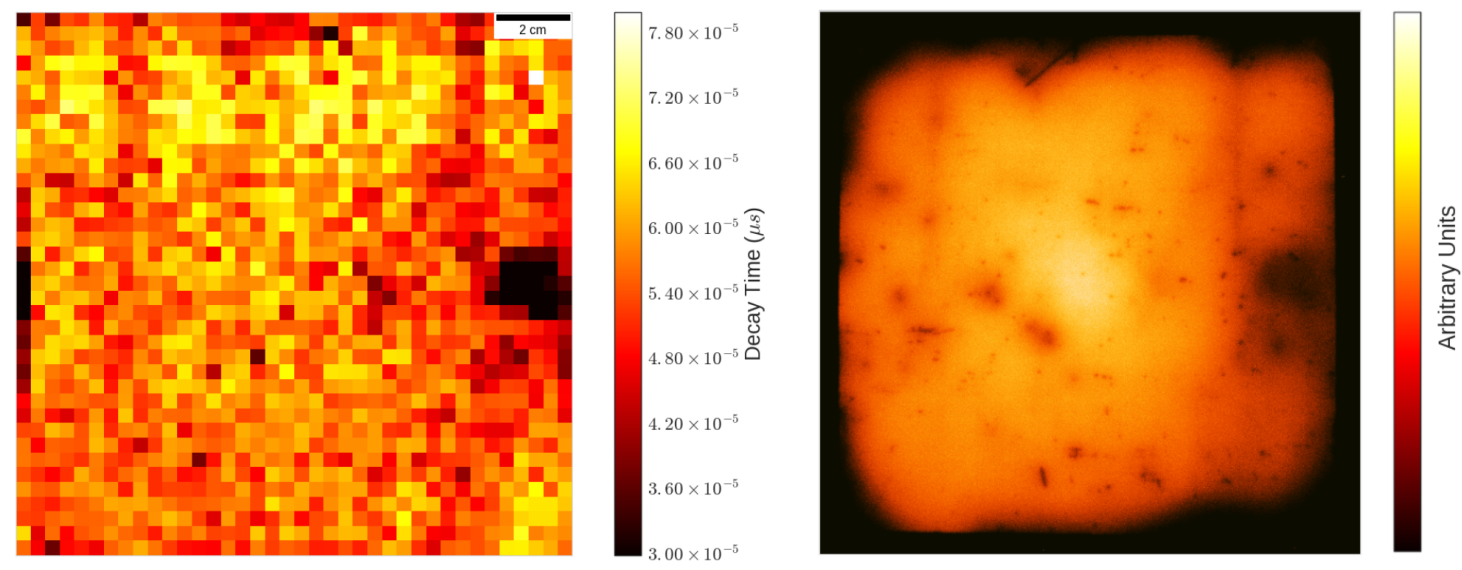

Figure 6.12: Decay \& PL Comparison: We compare a PL and time-resolved decay map for Sample 1 in this figure to identify cell specific defects. Sample 1 is quite noisy across the entire map. The measured decay times are pushing the limits of the system resolution. One defect along the right edge of the sample does clearly track the defect uncovered in the PL image.

\section{Sample 2}

Average decay time across the sample 2 shown in Figure 6.13 is on the order of $65 \mu \mathrm{s}$. The prominent point defect at the top center of the decay map corresponds to an underperforming region in the PL image. The vertical line along 
approximately $1 / 3$ rd of the width away from the right hand edge also corresponds to defects in the PL image. This defect looks like similar track defects observed earlier.
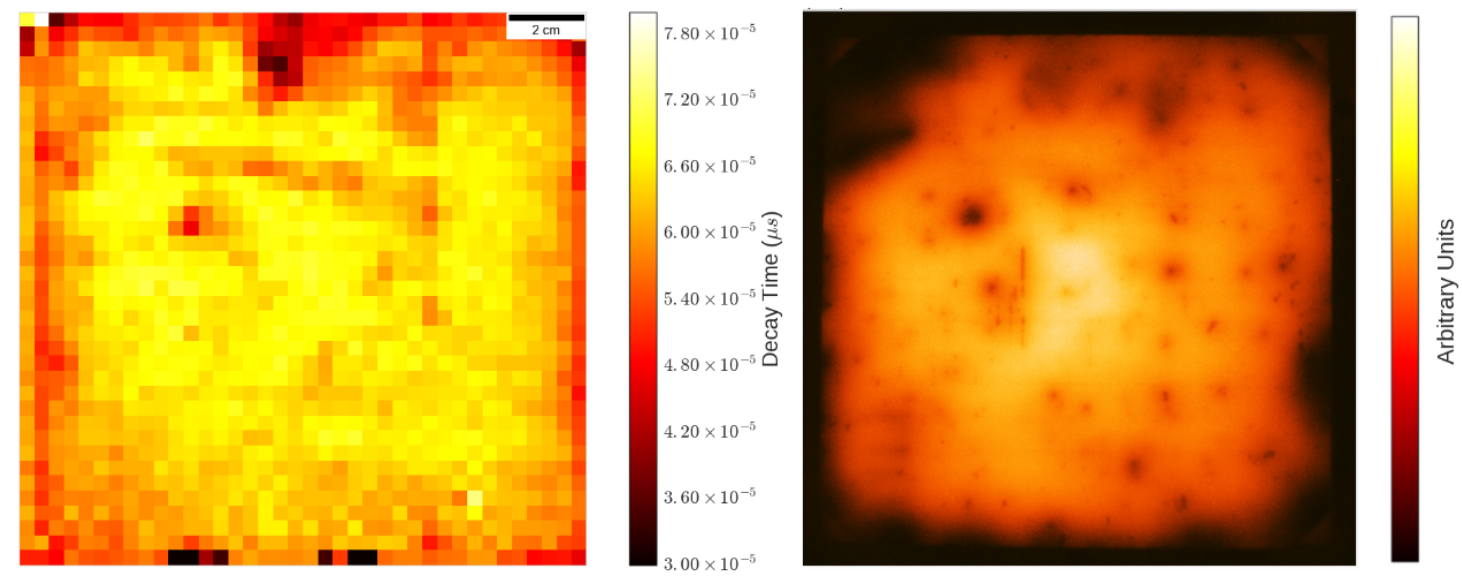

Figure 6.13: Comparison of PL and time-resolved decay map for Sample 2.

\section{Sample 5}

The scratched region at the bottom edge of the sample appears more prominently in the decay map than the severely defective blob defect near the top of the sample. Notice that the defects encroaching from the left hand side of the sample are not visible in the microwave detected decay map. 

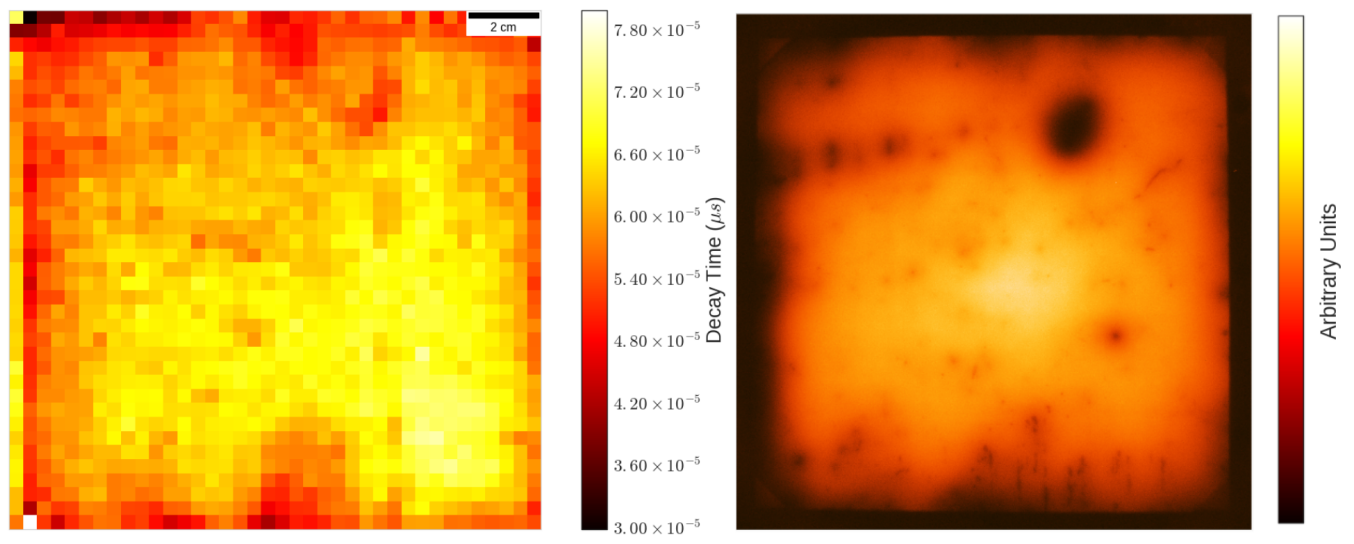

Figure 6.14: Comparison of PL and time-resolved decay map for Sample 5. 


\subsection{High Resolution Defect Scan}

High resolution defect scans of a damaged portion of a solar cell are possible using the microwave technique. Photoluminescence images illustraed in Figure 6.15 show a great number of scratches working towards the center of the cell. Several larger point defects are visible as well in both the PL image and microwave reflectance maps.
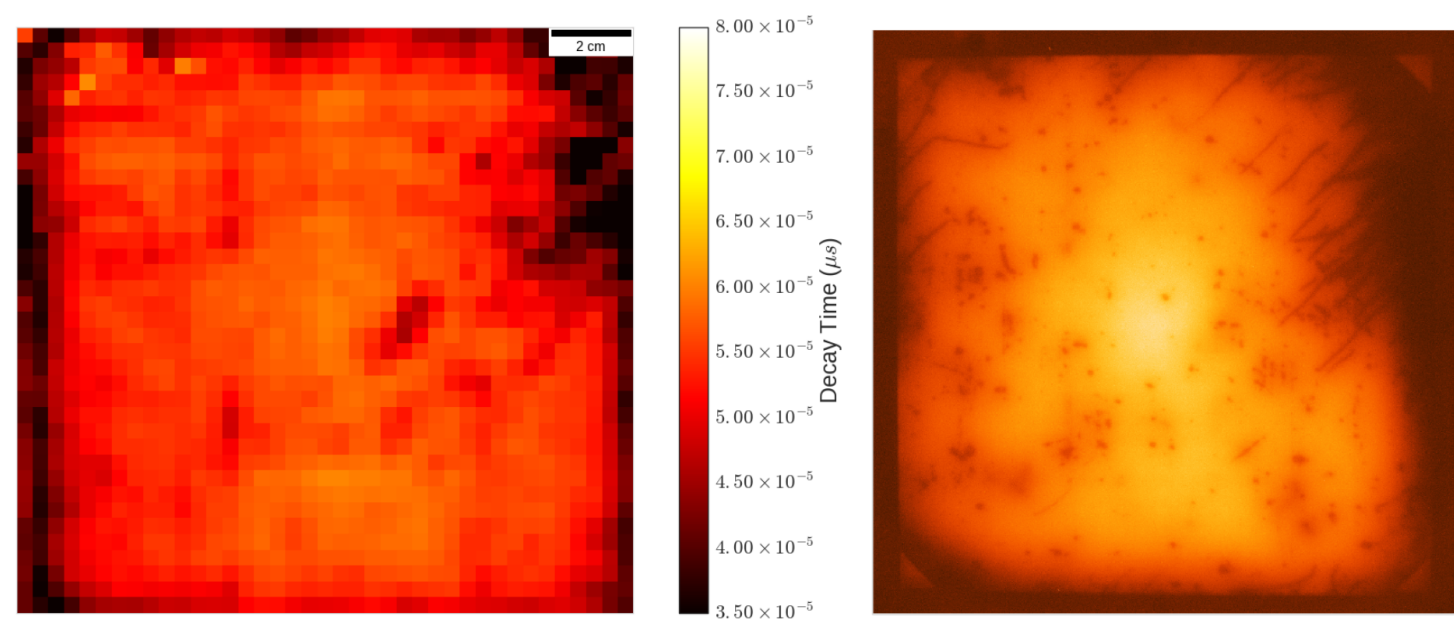

Figure 6.15: Comparison of photoluminescence and microwave reflectance amplitude data. The photoluminescence data was taken at $1 \mathrm{MP}$ resolution with a relatively weak excitation source. The map is recorded using a $4 \mathrm{~mm}$ step size along both the $\mathrm{x}$ and $\mathrm{y}$ axes.

Now that a defective region has been found, we can zoom and take a longer high resolution map of the affected area. The step size has been reduced to a maximum of $500 \mu m$ and the laser beam was reduced to a point. Although knife edge measurements of the laser spot size were not performed, we expect the spot size to be considerably less than the $500 \mu m$ step size. 

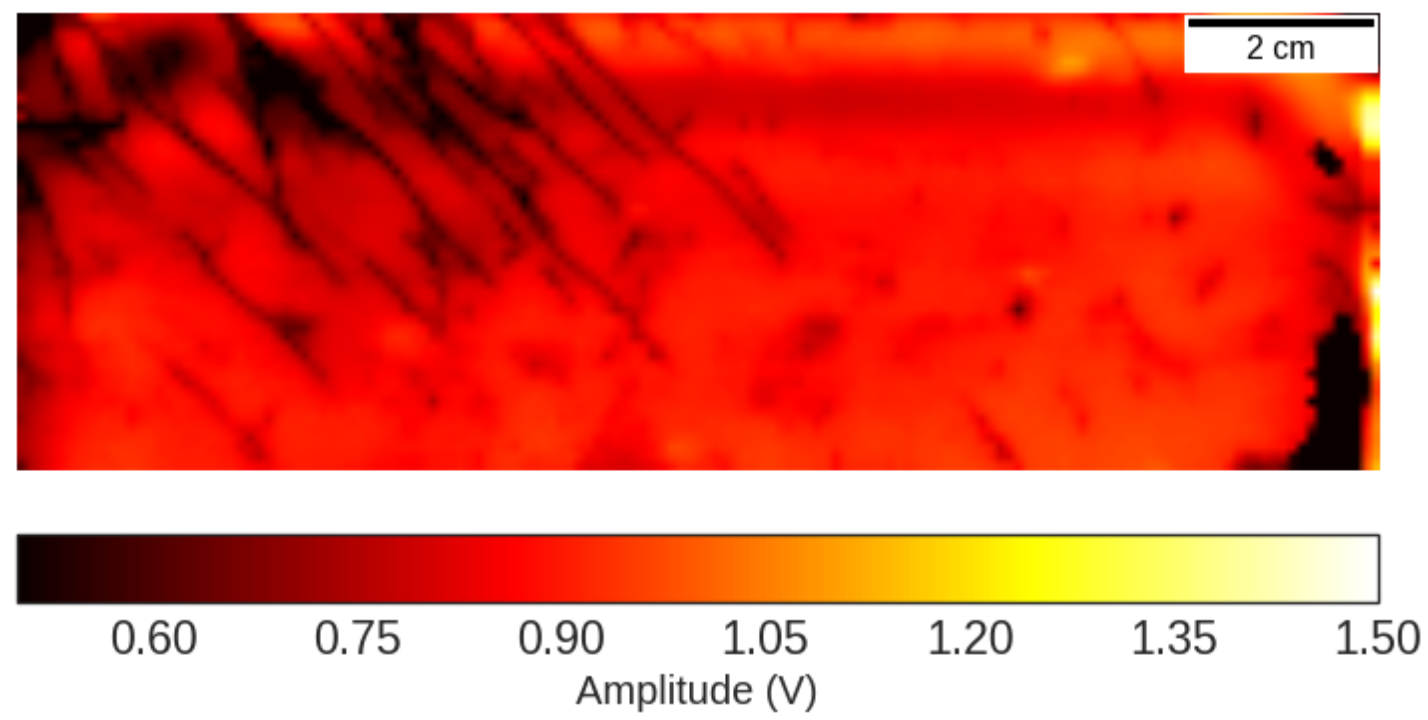

Figure 6.16: A high resolution scan of the defective corner shown in Figure 6.15 shows many distinct scratches running over the surface of the sample coinciding with the affected areas in the corresponding photoluminescence image.

\subsection{Application of High Dielectric Probe}

One of the more interesting and unexpected measurements that proved extremely useful was the idea of using high dielectric sensor pads to help couple microwave energy to the sample. Richmond and Tice [3] first used these probes to improve the accuracy of their $\mathbf{E}$ field measurements. The application of a high dielectric material lowers the cutoff frequency of the circular waveguide. Our purpose in deploying these pads is to reduce the area visible to the microwave receiver so that we are capable of measuring right up to the edge of the sample if needed. This allows us to create a well defined microwave spot size on the surface of the sample that is smaller than that provided by either the lens or free space measurements. A smaller probe has the added benefit of being able to more accurately characterize the edges of the solar cell. 
This effect should not be surprising since the the cutoff frequency for the lowest circular waveguide modes is calculated from

$$
f_{c}=\frac{p_{11} c}{2 \pi a \sqrt{\epsilon_{r}}}
$$

where $p_{11}=1.841$ for $T E_{11}, p_{11}=2.405$ for $T M_{01}$ and the dependence on $\epsilon$ is shown.

Figure ?? illustrates the three probes tested for high resolution measurements in this thesis. The probe on the left is made of alumina with $\epsilon_{r} \sim 10$ while the polyacryllic rod in the middle has $\epsilon_{r} \sim 4.7$. The right hand probe also uses alumina, but is much smaller $(5 \mathrm{~mm})$ and functions to help couple energy from the resonant cavity to the sample.

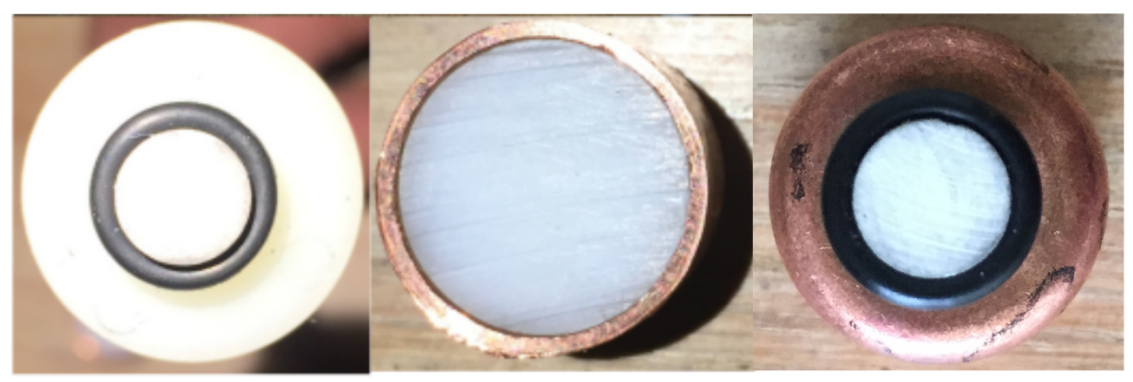

Figure 6.17: This figure shows three different probe styles applied directly underneath the sample. The three probe sizes are $5 \mathrm{~mm}, 7 \mathrm{~mm}$, and $13 \mathrm{~mm}$.

Circular Waveguide $f_{c}$ for Dielectric Probes

\begin{tabular}{lllllc}
\hline Probe Size & Aperture Radius & $\epsilon_{r}$ & Material & $f_{c}$ for $T E_{11}$ & $f_{c}$ for $T M_{01}$ \\
\hline $5 \mathrm{~mm}$ & $2.6 \mathrm{~mm}$ & 10.4 & Alumina & $10.5 \mathrm{GHz}$ & $13.7 \mathrm{GHz}$ \\
$7 \mathrm{~mm}$ & $3.5 \mathrm{~mm}$ & 10.4 & Alumina & $7.8 \mathrm{GHz}$ & $10.5 \mathrm{GHz}$ \\
$12.7 \mathrm{~mm}$ & $6.65 \mathrm{~mm}$ & 4.7 & polyacryllic & $6.5 \mathrm{GHz}$ & $8.5 \mathrm{GHz}$ \\
\hline
\end{tabular}

Table 6.3: Table of measured probes and the fundamental mode cutoff frequency for a circular waveguide. 
All three probes proved sufficient for the task of limiting the view of the $\mathrm{RF}$ receiver to the intended spot size. A test with and without the dielectric inserted for probe \#3 on the right side of Figure ?? also proved that the dielectric allows longer wavelengths to squeeze through the narrower aperture that would otherwise not be possible. Probe \#3 was used to collect data for all of the decay map measurements.

\subsection{Summary of Reference Images}

This section is simply used to group all of the PL photos together in Figure 6.18 for quick reference.
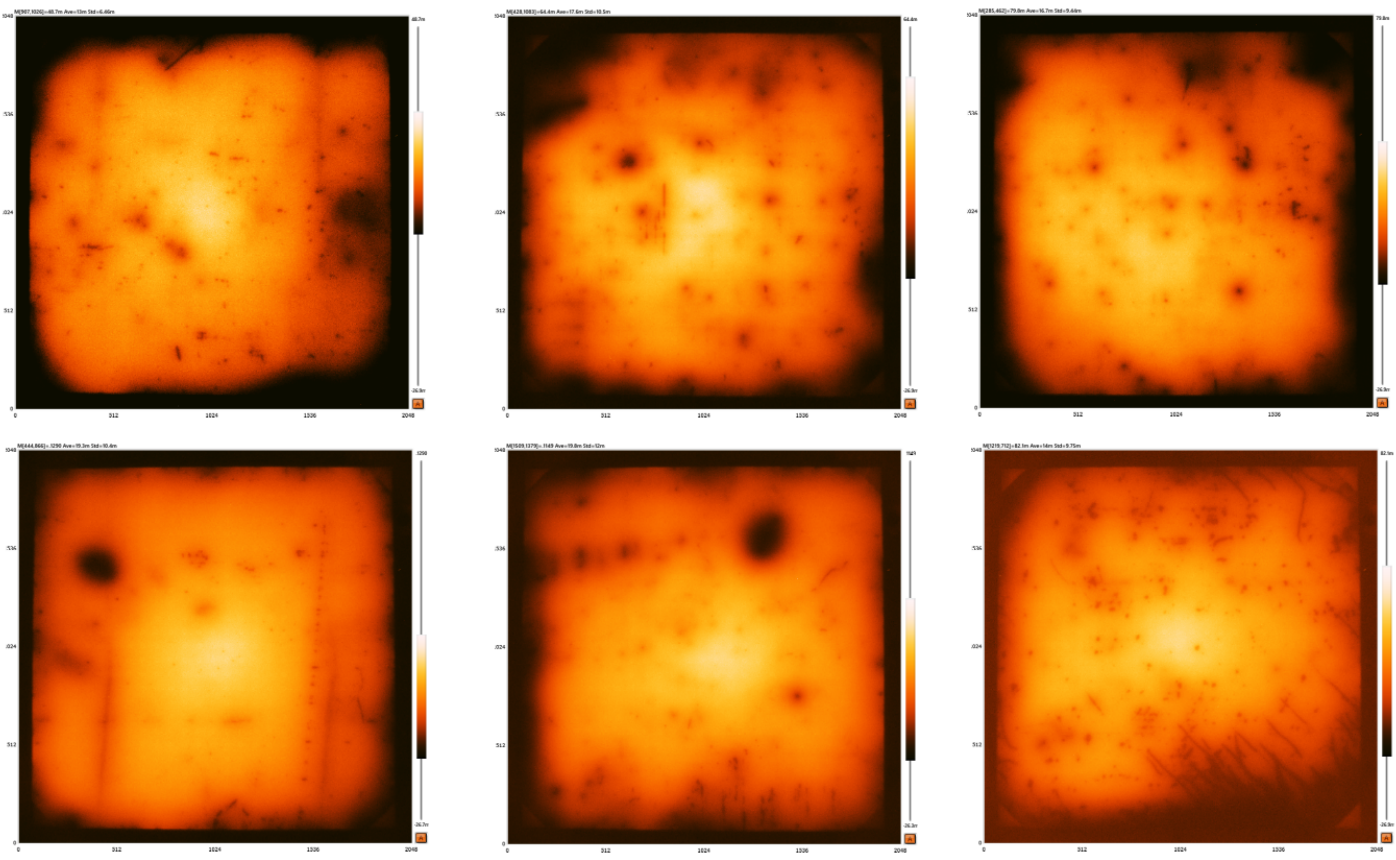

Figure 6.18: Summary of reference photoluminescence images for the sample set. Samples were biased to approximately 1 Sun with a pair of high power $528 \mathrm{~nm}$ (green) LEDs. Top Row: Samples \#1,\#2,\#3; Bottom Row: Samples \#4, \#5, \#10 


\subsection{Summary of Decay Maps}

This section is simply used to group all of the decay maps together in Figure 6.19 for quick reference.
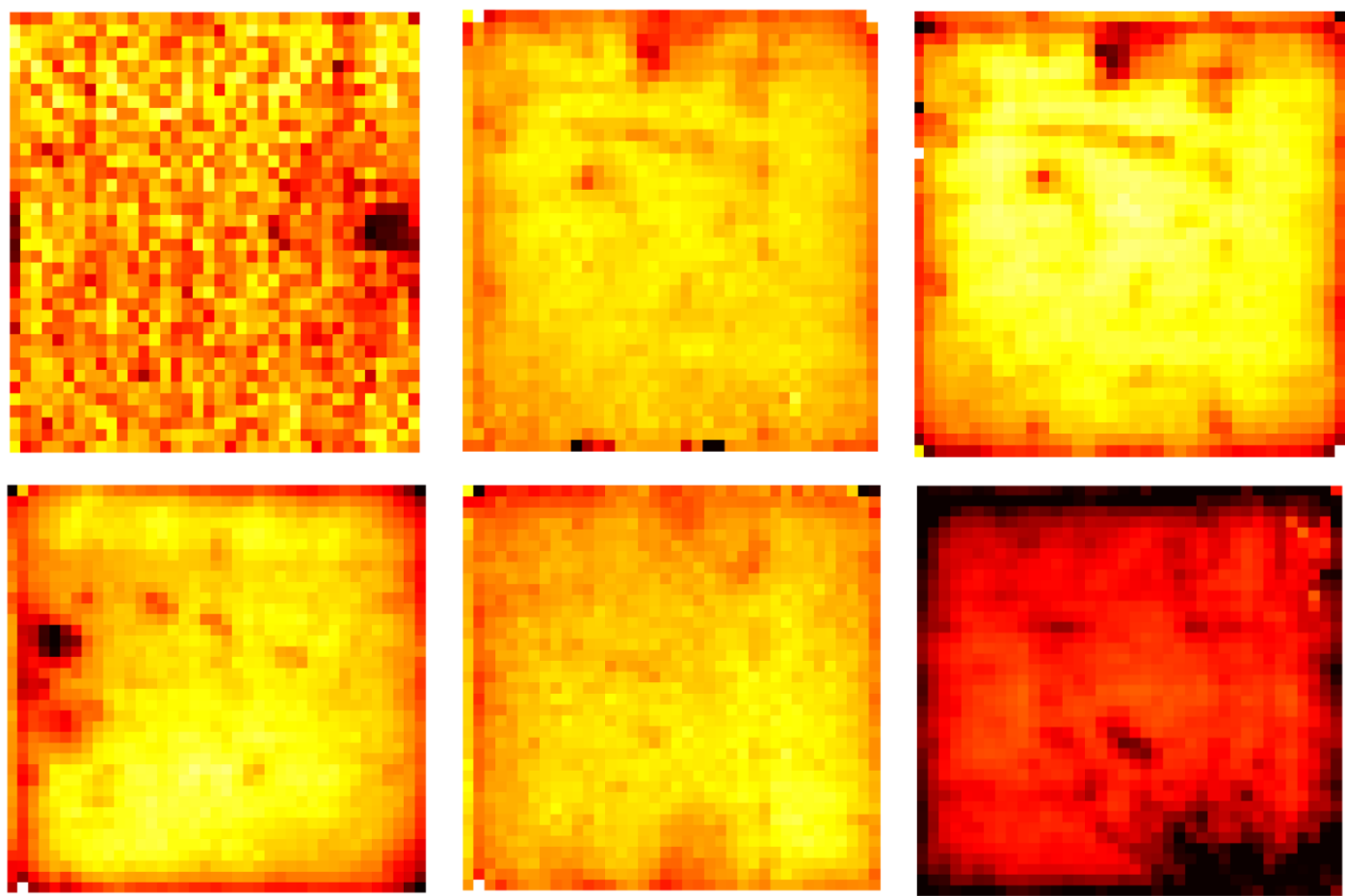

Figure 6.19: Summary of time-resolved decay maps. All decay times were calculated using the Levenberg-Marquardt exponential fit model. The signal-to-noise ratio of Sample 1 proved too small to reliably extract meaningful minority-carrier decay information. Samples 2-5 each recorded consistent decay constants on the order of $75 \mu \mathrm{s}$. Measurements on sample 10 recorded a slightly lower decay time constant of approximately $55 \mu$ s. Top Row: Samples \#1,\#2,\#3; Bottom Row: Samples \#4, \#5, \#10 


\section{Conclusion}

In this thesis, we focused on the development of a non-contact metrology technique capable of determining both the quantum efficiency and minority-carrier lifetime of a solar cell prior to the surface metalization phase of processing. A slightly different way of thinking about the problem is presented using the modulated scatterer concept from antenna theory in addition to the widely applied impedance mismatch concept found in solar cell and semiconductor metrology papers. Reflectometer measurements of the quantum efficiency and minority-carrier decay of silicon solar cells are checked against the well behaved response under various optical filters, JV operating point dependence on the small-signal microwave reflectance and measurement repeatability across multiple samples.

After reviewing the results in the previous chapter against the claims made at the beginning of this work, we are unable to definitively conclude that the system is capable of extracting minority-carrier lifetime. It is true that the measurement is sensitive to changes of carrier concentration on the 1-2 $\mu$ s time scale associated with the surface lifetime. However, additional studies probing the effect of that the non-uniform distribution of carriers has on effective lifetime following the work of [22] is required. Unlike the QE technique where a number of device related tests affirmed the validity of the measurement, we do not have a corresponding set of tests that can be used to validate the lifetime measurement. A second measurement technique is needed to confirm the 
measured results. With more data, it may be possible to use this technique to extract minority-carrier lifetime in the future.

The body of work performed in this thesis is believed to advance both inline and laboratory grade solar metrology systems in the following areas:

- The development of a non-contact measurement technique capable of reporting the spectral performance of a solar cell prior to the surface metalization phase of processing where physical contact is not possible. This technique facilitates the spectral binning of solar cells as part of the manufacturing line process control loop. The use of an entirely solid-state light engine also allows the measurement to be performed almost $100 \mathrm{x}$ faster per measurement site than traditional contact quantum efficiency measurements. The massive improvement in measurement speed now makes in-line measurements feasible.

- A different approach to the conceptual problem is presented using the modulated scatterer concept widely applied in antenna theory problems. We believe that much of the work found in the modulated scattering literature have largely gone unnoticed in the semiconductor characterization industry. We hope that this observation will lead to future studies relating the two fields. Concepts from antenna theory that are particularly relevant are the antenna reciprocity theorem and aperture antenna field patterns.

- Unlike many semiconductor measurement techniques available today, this work focuses on performing the measurement in the far-field. The 
application of high dielectric sensor pads and/or metamaterial lenses capable of achieving better than $\lambda / 4$ spatial resolution allow for small spot size measurements with low insertion loss. An item of more significance though is the ability to perform full cell measurements by illuminating the entire cell with an aperture antenna.

\subsection{Future Work}

While we do not consider the fabrication of a low cost 3D printed microwave antennas to be of much significance by way of the goals of this thesis, we feel that the topic is of considerable interest and should be pursued in a future study. The results of this thesis illustrate several potential avenues of additional study.

1. The work presented in this thesis focused heavily on the development of instrumentation capable of capturing the desired quantum efficiency data. A prolonged study of the relationship between the impedance mismatch formulation of the problem and the modulated scatterer formulation would greatly benefit the field as a whole. The justification here is our demonstration of performing the measurements in the far field rather than the commonly used near field. As a far-field measurement antenna concepts will play a significant role in the measurement. To this end, measurements of both the near and far-field antenna pattern would demonstrate the concept of a solar cell aperture antenna and go a long way towards relating the two approaches. 
2. Tracking the time-resolved motion of charge carriers in the junction of the solar cell from both the amplitude and phase information of the reflectance signature is another potential application. In the model presented in this thesis, we assumed that the reflection in the sample was only due to the highly doped emitter layer. However, this is clearly not accurate enough since we can detect carrier concentrations throughout the solar cell using the blue and red response. A more sensitive waveguide receiver may be sensitive enough to detect the relative phase shift between a surface blue response and a red back surface response.

3. Non-contact quantum efficiency measurements of thin film CdTe solar cells and multi-junction samples have yet to be conducted. The case for multi-junction solar cells is particularly interesting because the microwave technique could detect the photogenerated charge carriers located in a middle junction without the application of special bias considerations required by the series connected diode elements. Very-high efficiency multi-junction samples are often used in space-grade high performance and high reliability applications. The introduction of a non-destructive technique to characterize the quantum efficiency of the devices without physical contact might be seen as a significant improvement over conventional methods.

4. We would also like to see this work extended to include quantum efficiency measurements between the fingers of metalized samples. Now that we have data indicating the measurement signal-to-noise ratio, it is now possible to determine how many more photons are required to be injected if the spot size were limited to only $1-2 \mathrm{~mm}$. Such a measurement 


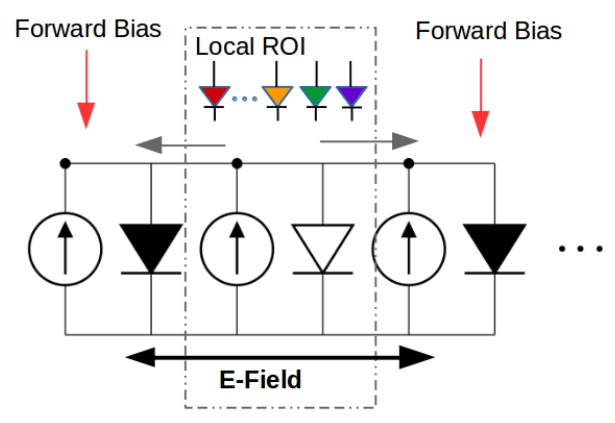

Figure 7.1: A localized short-circuit current experiment using bias lasers to suppress the junction of the device

would allow the samples to be tested throughout the entire process using a single measurement.

5. The solar cell community standardized the QE measurement under shortcircuit dark illumination conditions. Figure 7.1 shows one experiment that would simulate a localized short-circuit bias condition at the point of measurement. The figure represents local view of a single solar cell as a collection of parallel devices. This experiment would short circuit all of the neighboring devices around the point of interest using high power lasers. However, the laser bias would not be introduced at the point of measurement. In this way, the lateral currents at the measurement point are all forced to flow away towards towards the compressed junctions of its neighbors. Therefore, all current is efficiently removed from the localized region and thus resembles a short-circuit current measurement. 


\section{References}

[1] R. Justice and V. Rumsey, "Measurement of electric field distributions," IRE Transactions-Antennas and Propagation, December 1954.

[2] J. Richmond, "A modulated scattering technique for measurement of field distributions," IRE Transactions-Microwave Theory and Techniques, 1955.

[3] J. Richmond and T. Tice, "Probes for microwave near-field measurements," IRE Transactions: Microwave Theory and Techniques, p. 32, April 1955.

[4] R. N. Hall, "Electron-hole recombination in germanium," Phys. Rev., vol. 87, p. 387, July 1952.

[5] W. Shockley and W. Read, "Statistics of the recombinations of holes and electrons," Phys. Rev., vol. 87, pp. 835,842, September 1952.

[6] W. M. Bullis, "Measurement of carrier lifetime in semiconductors-an annotated bibliography covering the period 1949-1967," National Institute of Standards and Technology (NIST, formerly National Bureau of Standards), Tech. Rep., November 1968, technical Note: 465.

[7] A. Ramsa, H. Jacobs, and F. Brand, "Microwave techniques in measurement of lifetime in germanium," J. Appl. Phys., vol. 30, pp. 1054-1060, 1959.

[8] S. Dev and B. Nag, "Measurement of lifetime of carriers in semiconductors through microwave reflection," J. Appl. Phys., vol. 33, p. 1604, April 1962. 
[9] H. Jacobs, F. brand, J. Meindl, S. Weitz, R. Benjamin, and D. Holmes, "New microwave techniques in surface recombination and lifetime studies," Proc. IEEE, vol. 51, pp. 581-592, April 1963.

[10] J. Bhar, "Microwave techniques in the study of semiconductors," Proc. IEEE, vol. 51, pp. 1623-1631, November 1963.

[11] M. Kunst and G. Beck, "The study of charge carrier kinetics in semiconductors by microwave conductivity measurements," J. Applied Physics, vol. 60, pp. 3358-3566, Nov 1986.

[12] — " "The study of charge carrier kinetics in semiconductors by $\mathrm{mi}-$ crowave conductivity measurements. ii," J. Appl. Phys., vol. 63, p. 1093, 1988.

[13] M. Schofthaler and R. Brendel, "Sensitivity and transient response of microwave reflection measurements," Journal of Applied Physics, vol. 77, April 1995.

[14] R. K. Ahrenkiel and S. W. Johnston, "Interaction of microwaves with photoelectrons in semiconductors," J. Vacuum Science E Technology B, vol. 26, pp. 1508-1515, 2008.

[15] J. D. Dyson, "Measurements of near fields of antennas and scatterers," IEEE: Transactions on Antennas and Propagation, vol. 21, no. 4, p. 446, July 1973.

[16] V. G. Veselago, "The electrodynamics of substances with simultaneously negative values of permittivity and permeability," Sov. Phys.-Ups., vol. 10, p. $509,1968$. 
[17] R. Shelby, D. Smith, and S. Schultz, "Experimental verification of a negative index of refraction." Science, vol. 292, no. 5514, pp. 77 - 79, 2001. [Online]. Available: http://stats.lib.pdx.edu/proxy.php?url=http: //search.ebscohost.com.proxy.lib.pdx.edu/login.aspx?direct=true\&db= aph\&AN=4364147\&site=ehost-live

[18] J. Pendry, "Negative refraction makes a perfect lens," Physical Review Letters, vol. 85, p. 3966, 2000.

[19] C. Simovski and S. Tretyakov, "Historical notes on metamaterials," in Theory and Phenomena of Metamaterials, F. Capolino, Ed. Boca Raton, FL: CRC Press/Taylor \& Francis, 2009, ch. 1, pp. $1.1-1.17$.

[20] I. Ehrenberg, S. Sarma, and B.-I. Wu, "A three-dimensional self-supporting low loss microwave lens with a negative refractive index," J. Appl Phys., vol. 112, 2012.

[21] G. Beck and M. Kunst, "Contactless scanner for photoactive materials using laser-induced microwave absorption," AIP: Review of Scientific Instruments, vol. 57, p. 197, February 1986.

[22] C. Swiatkowski, A. Sanders, K. Buhre, and M. Kunst, "Charge-carrier kinetics in semiconductors by microwave conductivity," Journal of Applied Physics, vol. 78, p. 1763, August 1995.

[23] K. Iizuka, "Photoconductive probe for measuring electromagnetic fields," PROC. Institute of Electrical Engineers, vol. 10, p. 1747, October 1963. 
[24] A. L. Cullen and J. C. Parr, "A new perturbation method for measuring microwave fields in free space," PROC. Institute of Electrical Engineers, vol. 102B, pp. 836-844, November 1955.

[25] A. M. Vural and D. K. Cheng, "A light-modulated scattering technique for diffraction field measurements," Radio Science, vol. 68D, April 1964.

[26] A. Vural, D. K. Cheng, and B. J. Strait, "Measurement of diffraction fields of finite cones by a scattering technique using light modulation," IEEE Transactions on Antennas and Propagation, March 1962.

[27] R. Garbacz and J. Eberle, "The measurement of time-quadrature components of a scattered field," in WESCON/60 Conference Record, Aug. 1960.

[28] M.-K. Hu, “On measurements of microwave $\bar{E}$ and $\bar{H}$ field distributions by using modulated scattering methods," IRE Transactions on Microwave Theory and Techniques, August 1959.

[29] B. J. Strait and D. K. Cheng, "Measurement of microwave magnetic diffraction fields," IEEE Transactions on Antennas and Propagation, April 1962.

[30] J. Kraus and R. Marhefka, Antennas for All Applications, third edition ed. San Fransisco, California: McGraw-Hill, 2002.

[31] S. Ballantine, "Reciprocity in electromagnetic, mechanical, acoustical, and interconnected systems," Proceedings of the Institute of Radio Engineers, vol. 17, no. 6, pp. 929-952, 1929.

[32] W. L. Stutzman and G. A. Thiele, Antenna Theory and Design, 2nd ed. USA: John Wiley \& Sons, Inc., 1998. 
[33] S. J. Orfanidis, Electromagnetic Waves and Antennas, first edition ed. Piscataway, New Jersey: ECE Department: Rutgers University, 2016.

[34] D. L. Young, B. Egaas, S. Pinegar, and P. Stradins, "A new real-time quantum efficiency measurement system," Photovoltaic Specialists Confernce, vol. 33, May 2008. 


\section{Appendix A: Electroplating Process}

Two metalization processes used in this thesis to metalize 3D printed objects are described in detail. Both methods are found to be equally valid but have slightly different advantages depending on the geometry and size of the object being metalized. Experimentation with various waveguides, horns and lenses has allowed the author to determine the following rules of thumb. When possible Method 2 as it provides the quickest results and lowest initial surface impedance. Sometimes Method 2 is not always feasible due to an obscured surface area. In that case, Method 1 can be used to form a preconductive layer on the obscured surface. In all cases, the electroplating component of the process will preferentially coat locations of strongest $\mathbf{E}$ field termination. A custom field shaping probe may be required to coat difficult surfaces.

We should note here that we have only been able to successfully coat objects with large flat s urface a reas such a s the a ntenna and $m$ icrowave lens demonstrated earlier in this work. Work has been performed on $\mathrm{x}$-band waveguides and waveguide adapters but has yet to produce meaningful results using either of the two methods listed here. We are currently in the process of investigating a third technique in which the 3D printed object (assume waveguide), is sliced along the axis of a burried dimension that is difficult to $c$ oat. The part may now be coated using either Method 1 or Method 2 without difficulty. The current phase of work is attempting to seal the two pieces back together. With the help of a shaped $\mathbf{E}$ field it is believed to be possible to seal the two components together completely. If the thickness of the surface coating is more than several skin depths, then the discontinuity will be completely invisible to the interior of the waveguide.

\subsection{Method 1: Custom Process}

1. Remove Surface Contaminants: It is necessary to remove oils and surface residues prior to the coating process in order to achieve cohesion with the printed part. Warm soapy water works well for this step. 
2. Apply Shellac: A thin layer of shellac is applied on the surface of the part to be coated using a spray bottle mister. When exposed to air, the shellac forms an adhesive residue which helps the conductive layer adhere to the plastic part. We experimentally determined that the shellac is an innocuous enough adhesive layer that does not adversely alter the electroplating tank chemistry.

3. Add Copper Shavings: Before the shellac has dried, place the part in a container with fine copper shavings or particles. Shake the container to agitate the copper shavings so that it completely coats the part. All of our tests were performed using $60 \mu \mathrm{m}$ copper. Based on impedance measurements of the precoating layer, we recommed moving to $20-40 \mu \mathrm{m}$ size particles.

4. Remove Excess Copper: Gently remove excess copper shavings by taping the side of the part so all of the loose shavings fall off. Excess shavings will almost certainly lead to blotchy coatings. Furthermore, the extra thickness can cause the top layer to become solidified during the coating process but leave the layer underneath untouched. This can lead to layers flaking off during the drying process.

5. Remove Oxides: An acid bath is used to strip away the surface oxides on the copper shavings in order to improve conductivity across the part. The acid bath is also used to remove any additional oils that may have been introduced between steps 1-4. Our experiments used a bath containing 5\% hydrocloric acid and 95\% deionized water to acomplish this step. Note that extreme must be taken when mixing the hydrocloric acid bath as it will burn through most clothing and gloves. The acid also has an extreme exothermic reaction when added to the water. The acid should only be added a few drops at a time to prevent shattering or burnthrough.

6. Electroplate: Finally, the part is ready to be submerged in the electroplating tank for coating. Connect the cathode lead directly to the part. The anode is connected directly to a copper source submerged in the electroplating solution. When a potential is applied between the two leads, 
copper particles are ripped from the source and are deposited on the surface of the part. This process will take anywhere between $0.5 \mathrm{hr}$ to $24 \mathrm{hrs}$ depending on the geometry and size of the part.

7. Remove and clean part.

\subsection{Method 2: Airbrush Process}

1. Remove Surface Contaminants: It is necessary to remove oils and surface residues prior to the coating process in order to achieve cohesion with the printed part. Warm soapy water works well for this step.

2. Airbrush Paint: Copper paint is readily available for EMI reduction in PCB applications. An airbrush technique was found to produce the most uniform coatings across the entire surface of the object.

3. Electroplate: Same as above. 


\section{Appendix B: Poster Summary}

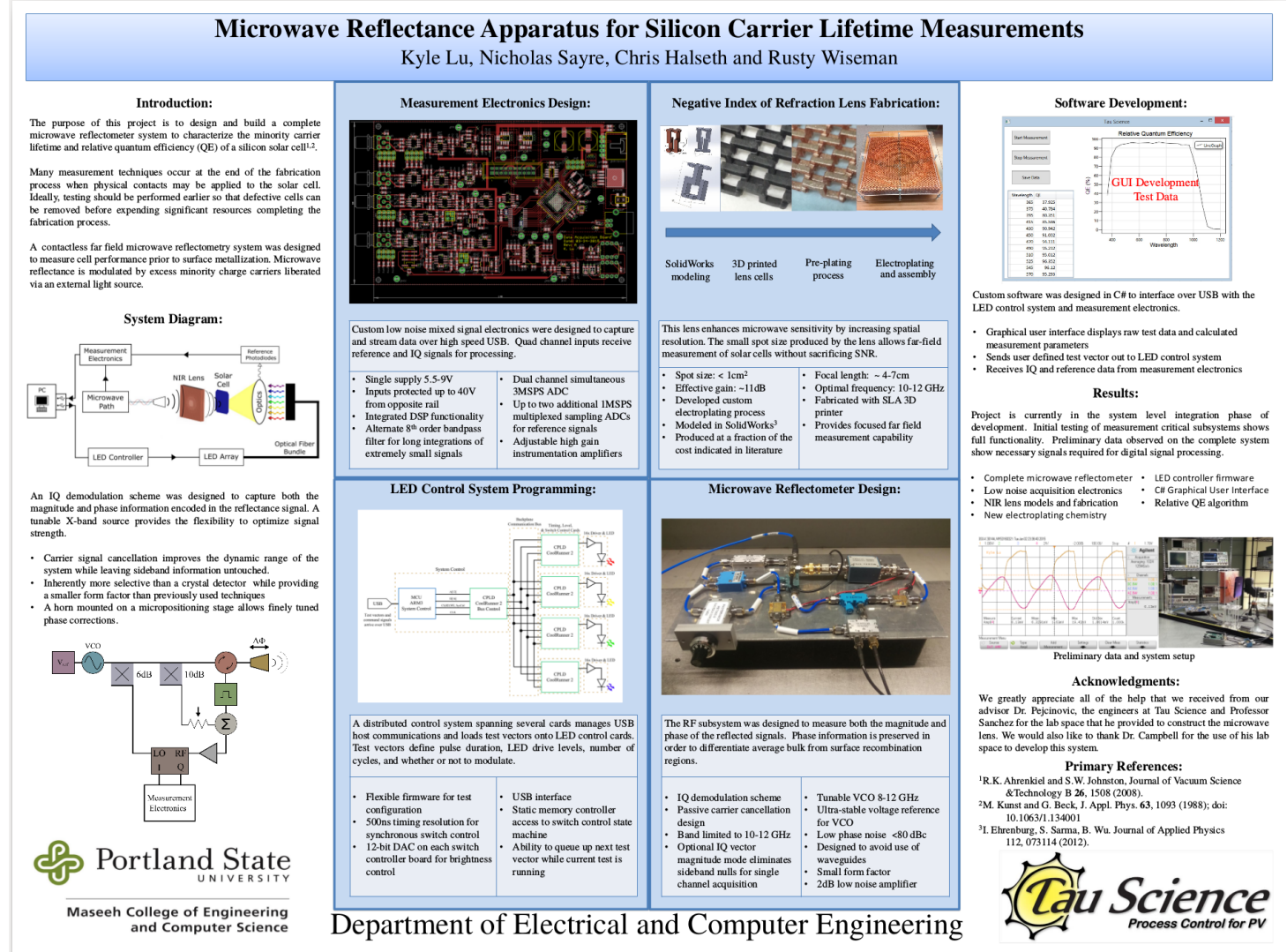

Figure 7.2: Team project for an industrial design course at Portland State University, 2015. 


\section{Appendix C: Measurement Configuration Settings}

\section{Application Configuration INI File}

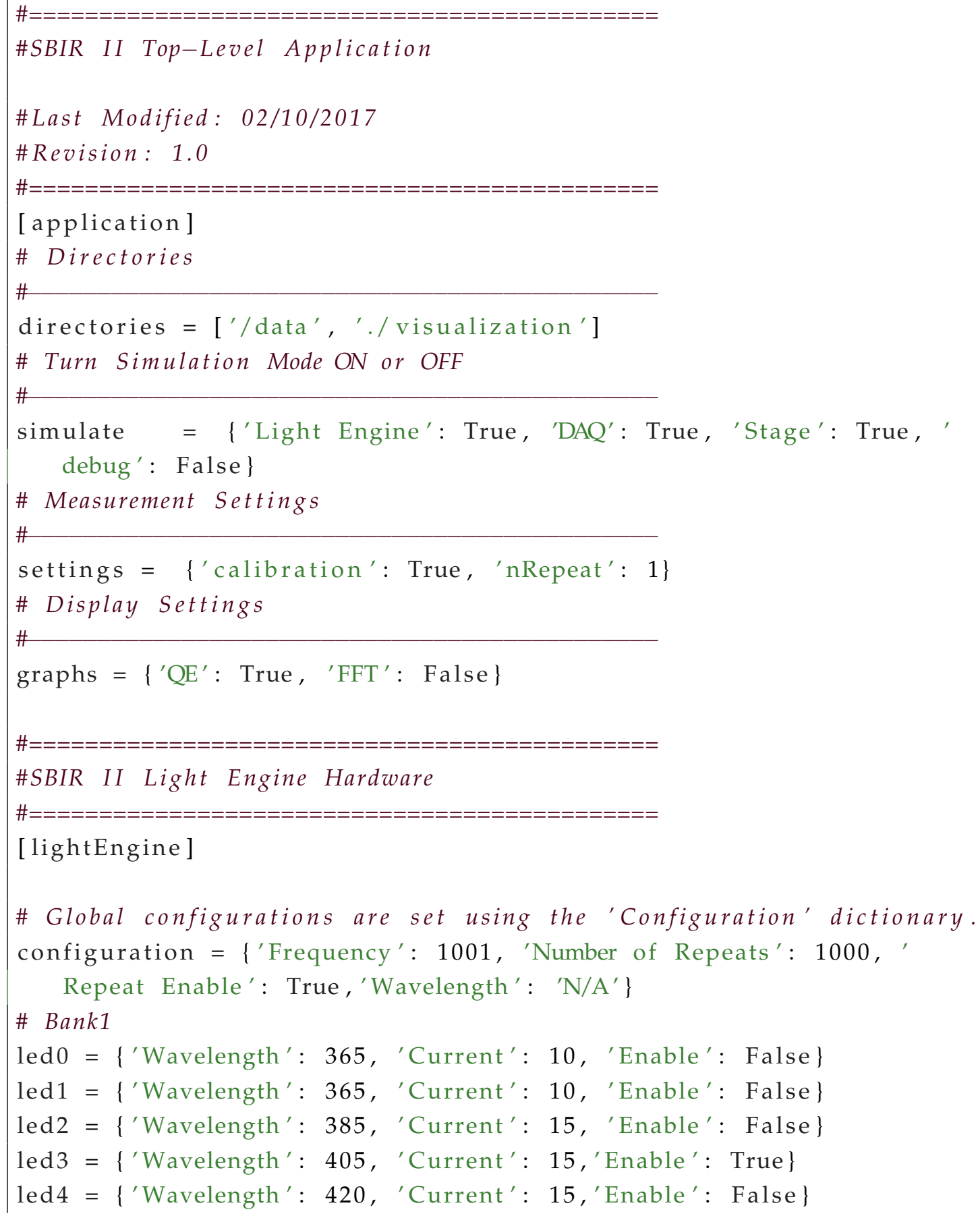




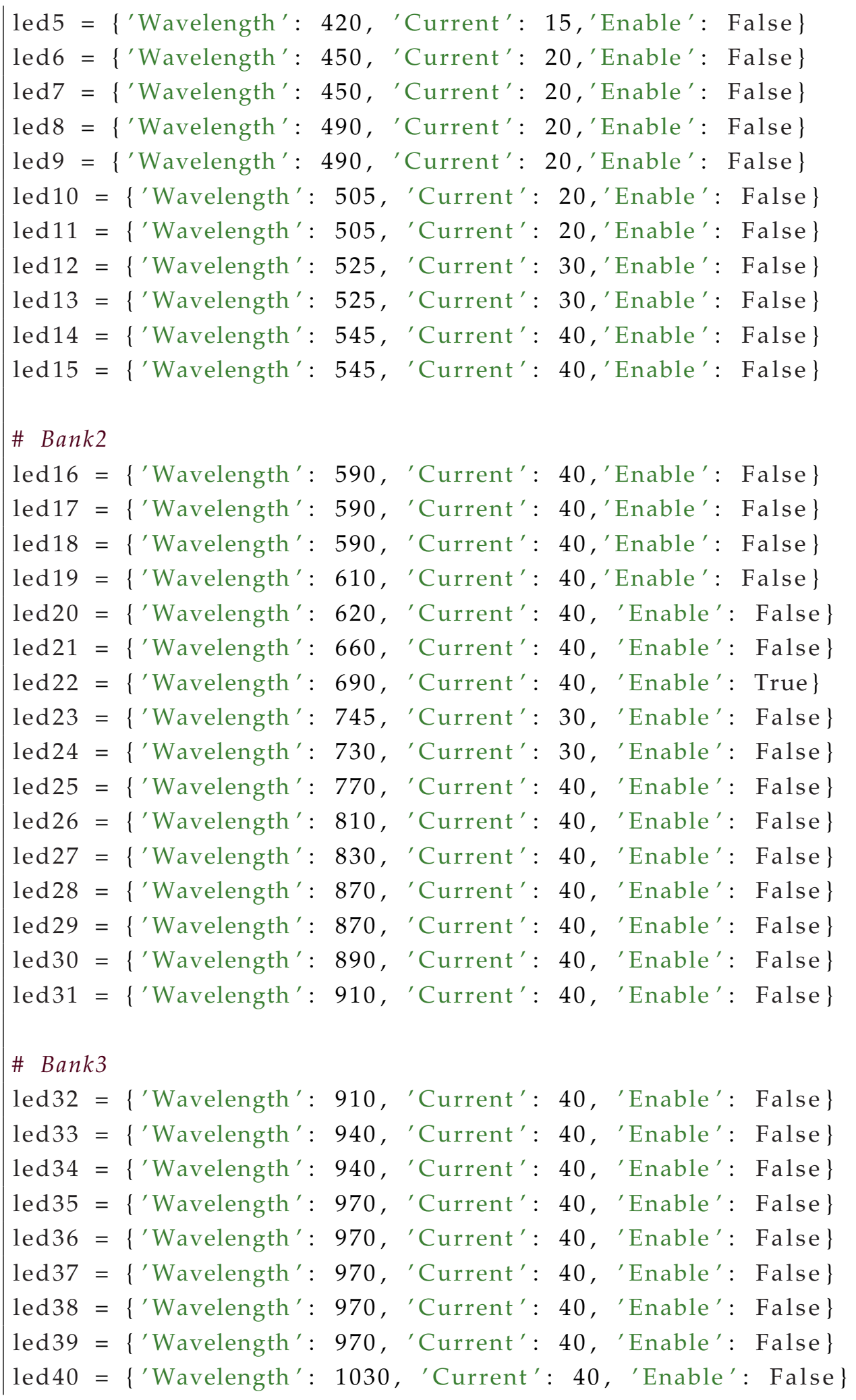




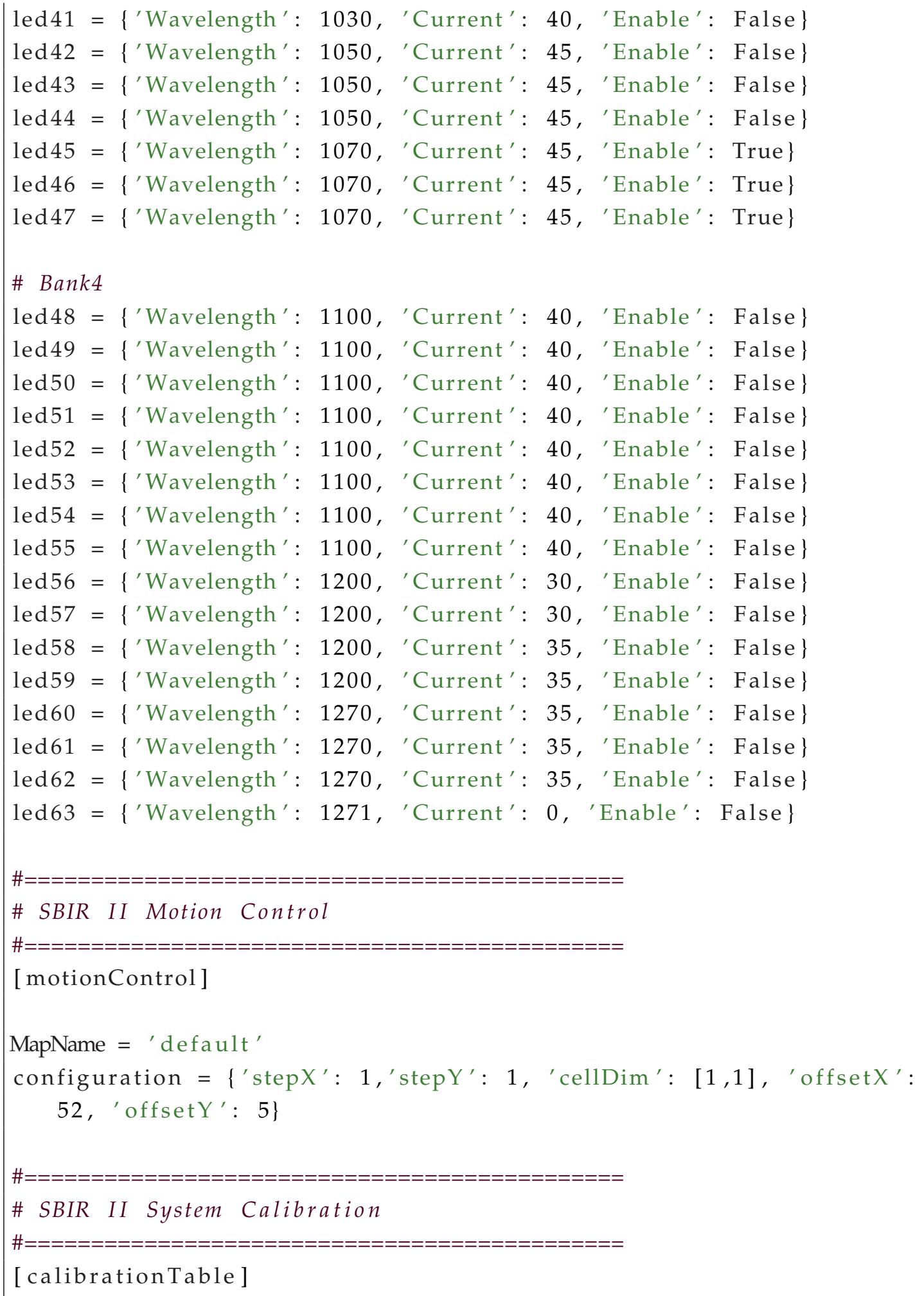


$\mathrm{xCalTable}=[365,375,385,405,420,450,470,490,505,525,545$, $610,625,710,730,750,810,825,850,900,920,950,970,990$, $1020,1050,1070,1100,1130,1160,1200,1250]$

yCalTable $=[45,60,70,75,85,85,85,85,85,85,85,85,85,85$, $85,85,85,85,85,85,85,85,85,85,83,75,40,25,15,10,8$, $5]$ 